\title{
Electron-deuteron deep-inelastic scattering with spectator nucleon tagging and final-state interactions at intermediate $x$
}

\author{
M. Strikman ${ }^{1, *}$ and C. Weiss ${ }^{2, \dagger}$ \\ ${ }^{1}$ Department of Physics, Pennsylvania State University, University Park, Pennsylvania 16802, USA \\ ${ }^{2}$ Theory Center, Jefferson Lab, Newport News, Virginia 23606, USA
}

(Received 28 June 2017; revised manuscript received 19 January 2018; published 27 March 2018)

\begin{abstract}
We consider electron-deuteron deep-inelastic scattering (DIS) with detection of a proton in the nuclear fragmentation region ("spectator tagging") as a method for extracting the free neutron structure functions and studying their nuclear modifications. Such measurements could be performed at a future electron-ion collider (EIC) with suitable forward detectors. The measured proton recoil momentum $(\lesssim 100 \mathrm{MeV}$ in the deuteron rest frame) specifies the deuteron configuration during the high-energy process and permits a controlled theoretical treatment of nuclear effects. Nuclear and nucleonic structure are separated using methods of light-front quantum mechanics. The impulse approximation to the tagged DIS cross section contains the free neutron pole, which can be reached by on-shell extrapolation in the recoil momentum. Final-state interactions (FSIs) distort the recoil momentum distribution away from the pole. In the intermediate- $x$ region $0.1<x<0.5$ FSIs arise predominantly from interactions of the spectator proton with slow hadrons produced in the DIS process on the neutron (rest frame momenta $\lesssim 1 \mathrm{GeV}$, target fragmentation region). We construct a schematic model describing this effect, using final-state hadron distributions measured in nucleon DIS experiments and low-energy hadron scattering amplitudes. We investigate the magnitude of FSIs, their dependence on the recoil momentum (angular dependence, forward/backward regions), their analytic properties, and their effect on the on-shell extrapolation. We comment on the prospects for neutron structure extraction in tagged DIS with an EIC. We discuss possible extensions of the FSI model to other kinematic regions (large/small $x$ ). In tagged DIS at $x \ll 0.1$ FSIs resulting from diffractive scattering on the nucleons become important and require separate treatment.
\end{abstract}

DOI: 10.1103/PhysRevC.97.035209

\section{INTRODUCTION}

Measurements of deep-inelastic lepton scattering (DIS) from nuclei with mass number $A>1$ address several key topics in short-range nuclear structure and quantum chromodynamics $(\mathrm{QCD})$. One is the partonic structure of the neutron, which is needed for the flavor decomposition of the nucleon's valence and sea quark densities and for the separation of singlet and nonsinglet nucleon structure functions in studies of the scale dependence (QCD evolution, higher-twist effects). Another topic is the modifications of the nucleon's partonic structure in the nucleus and their dependence on the scaling variable $x$ (EMC effect at $x>0.3$, antishadowing at $x \sim 0.1$ ), which attest to the presence of non-nucleonic degrees of freedom in nuclei and reveal the QCD structure of nucleonnucleon interactions [1,2]. Yet another topic is coherence phenomena at $x \ll 0.1$ such as nuclear shadowing, which

\footnotetext{
*strikman@phys.psu.edu

†weiss@jlab.org
}

Published by the American Physical Society under the terms of the Creative Commons Attribution 4.0 International license. Further distribution of this work must maintain attribution to the author(s) and the published article's title, journal citation, and DOI. Funded by $S C O A P^{3}$. arise from the participation of multiple nucleons in the DIS process and govern the approach to the unitarity limit at high energies [3]. Experiments in nuclear DIS have been carried out in fixed-target $e A / \mu A$ scattering at several facilities (SLAC, HERMES, CERN EMC and COMPASS, FNAL E665, JLab; see Ref. [4] for a review) and will be extended further with the Jefferson Lab $12 \mathrm{GeV}$ upgrade. A much wider kinematic range would become accessible in colliding-beam experiments with a future electron-ion collider (EIC) [5-7]. A medium-energy EIC with a squared electron-nucleon center-of-mass (CM) energy $s_{e N} \equiv s_{e A} / A \sim 200-2000 \mathrm{GeV}^{2}$ would be ideally suited for nuclear DIS measurements in the region $x \gtrsim 10^{-3}$ and enable detailed studies of sea quarks and gluons in the nucleon and their nuclear modifications [8,9]. Complementary information is provided by measurements of hard processes in high-energy hadron and photon scattering on nuclei (RHIC, LHC) [10].

The main challenge in the analysis of nuclear DIS experiments is to account for the multitude of nuclear configurations that can be present in the initial state of the high-energy scattering process and affect its outcome [11]. The scattering can take place on any of the constituent protons and neutrons ( $p$ and $n$ ), in different states of their quantum-mechanical motion in the nucleus (momentum, spin). In addition, non-nucleonic degrees of freedom such as $\Delta$ isobars are excited by the nuclear binding. In the extraction of neutron structure one needs to isolate the DIS cross section arising from scattering on the neutrons and eliminate the effects of nuclear binding (Fermi motion, 
non-nucleonic degrees of freedom). For neutron spin structure one must also infer the effective polarization of the neutron in the polarized nucleus and account for the polarization of non-nucleonic degrees of freedom, particularly intrinsic $\Delta$ 's in polarized ${ }^{3} \mathrm{He}$ [12-14]. In the study of nuclear modifications at $x \gtrsim 0.1$ (EMC effect, antishadowing) one wants to relate the modifications to the nucleon interactions taking place in particular nuclear configurations (short-range correlations, exchange mechanisms). In traditional inclusive nuclear DIS measurements $e+A \rightarrow e^{\prime}+X$ these issues are addressed by modeling the nuclear effects for typical nuclear configurations and averaging over all possible configurations. The resulting theoretical uncertainty usually represents the dominant systematic error in neutron structure extraction. Likewise, this method provides limited possibilities for unraveling the dynamical origin of nuclear modifications. Major progress could come from experiments that provide information on the nuclear configurations present during the high-energy process through measurements of the nuclear final state.

Deep-inelastic scattering on the deuteron $(d, A=2)$ with detection of a nucleon $(N=p$ or $n)$ in the nuclear fragmentation region, $e+d \rightarrow e^{\prime}+N+X$, represents a unique method for performing DIS measurements in controlled nuclear configurations ("spectator tagging"). The nucleon emerges with a typical recoil momentum $\left|\boldsymbol{p}_{N}\right| \sim$ few $10-100 \mathrm{MeV}$ in the deuteron rest frame. ${ }^{1}$ At such momenta the deuteron's nonrelativistic $p n$ wave function is well known from low-energy measurements and can be used to construct the $p n$ light-front (LF) wave function entering in high-energy processes (see below). Because the deuteron has isospin $I=0, \Delta$ isobars in the wave function are strongly suppressed (they can occur only in $\Delta \Delta$ configurations), so that the deuteron can be treated as a $p n$ system for most of the configurations relevant to DIS [11]. Under these conditions the detection of the recoil nucleon and the measurement of its momentum positively identify the active nucleon and control its momentum during the DIS process. By measuring DIS with a tagged proton and extrapolating the measured recoil momentum dependence to the on-shell point near $\left|\boldsymbol{p}_{p}\right|=0$ (in the deuteron rest frame) one can eliminate nuclear binding effects and extract free neutron structure in a model-independent manner [15]. DIS on the deuteron with proton tagging was measured in the JLab CLAS BONuS experiment at $6 \mathrm{GeV}$ beam energy $[16,17]$ and will be explored further at $11 \mathrm{GeV}$ [18]. This setup covers recoil momenta $\left|\boldsymbol{p}_{p}\right| \gtrsim 70 \mathrm{MeV}$, which are larger than the typical nucleon momenta in the deuteron (the median of the nonrelativistic momentum distribution is $\sim 70 \mathrm{MeV})$. In such fixed-target experiments it is difficult to get slow protons (or neutrons) out of the target and measure their momenta with sufficient resolution, which restricts the measurements to large recoil momenta and prevents on-shell extrapolation.

Much more suitable for tagged DIS measurements are colliding-beam experiments, where the spectator nucleon moves on with approximately half the deuteron beam momentum and can be detected using forward detectors. Both

${ }^{1}$ We use units in which $\hbar=c=1$ and quote momenta in MeV/GeV.
EIC designs presently discussed include capabilities for forward nucleon detection [9,19-21]. The JLab EIC detector is designed to provide full coverage for spectator protons down to zero transverse momentum, and with a momentum resolution corresponding to $\left|\boldsymbol{p}_{p}\right| \sim 20 \mathrm{MeV}$ in the rest frame, as well as forward neutron detection. This setup would enable measurements of deuteron DIS with spectator tagging over the entire $\left(x, Q^{2}\right)$ range covered by the collider and thus permit extraction of neutron structure and study of nuclear modifications with control of the nuclear configuration. It would also allow for tagged measurements on the polarized deuteron, which is potentially the most precise method for determining neutron spin structure.

The theoretical analysis of tagged DIS on the deuteron relies essentially on the analytic properties of the scattering amplitude (and cross section) in the recoil proton momentum. As a function of the invariant four-momentum transfer between the deuteron and the recoiling proton, $t \equiv\left(p_{d}-p_{p}\right)^{2}$, the cross section has a pole at $t=M_{N}^{2}$ (we assume isospin symmetry and denote the common nucleon mass by $M_{N} \equiv$ $M_{p, n}$ ). The pole is contained in the impulse approximation (IA) amplitude and corresponds to the scattering from an on-shell neutron in the deuteron in unphysical kinematics. According to the general principles of scattering theory, the residue at the pole is given by the structure function of the free neutron. Nuclear binding and final-state interactions (FSIs) affect only the tagged deuteron structure functions away from the pole, at $t-M_{N}^{2} \neq 0$, not the residue at the pole. This makes it possible to extract the free neutron structure function, by measuring the proton-tagged DIS cross section as a function of $t$ and extrapolating to the on-shell point $t \rightarrow M_{N}^{2}$. In terms of the recoil momentum in the rest frame, $t-M_{N}^{2}=-2\left|\boldsymbol{p}_{p}\right|^{2}+$ $t_{\min }^{\prime}$, where $t_{\min }^{\prime}=-M_{d} \epsilon_{d}=-0.0041 \mathrm{GeV}^{2}\left(\epsilon_{d}=2.2 \mathrm{MeV}\right.$ is the deuteron binding energy), so that the on-shell point corresponds to unphysical values of the recoil momentum extremely close to zero, $\left|\boldsymbol{p}_{p}\right|^{2}=-t_{\text {min }}^{\prime} / 2$. The method is model independent and relies only on general properties of the tagged DIS cross section (analyticity, position of singularities). It has considerable theoretical appeal and can be turned into a practical tool, given sufficiently precise data at small recoil momenta.

Away from the nucleon pole, at $t-M_{N}^{2} \neq 0$, the recoil momentum dependence of the tagged DIS cross section is modified by FSIs. They result from amplitudes in which the final state produced in the DIS process on the active nucleon rescatters from the spectator nucleon and changes its momentum. They exhibit a complex dependence on the recoil momentum angle and magnitude, dictated by the kinematics of the rescattering process, and on $x$, because the character of the nucleon DIS final state changes as a function of the latter. The FSI effects in the tagged cross section need to be estimated quantitatively in order to assess the feasibility of neutron structure extraction through on-shell extrapolation. The same is needed in order to explore the possibility of separating initial-state nuclear modifications from final-state interactions in tagged DIS. Such an estimate requires a theoretical model of FSIs appropriate to the region of $x$ explored in the tagged DIS experiments.

In this article we develop the theoretical framework for tagged DIS measurements on the deuteron in the kinematic 


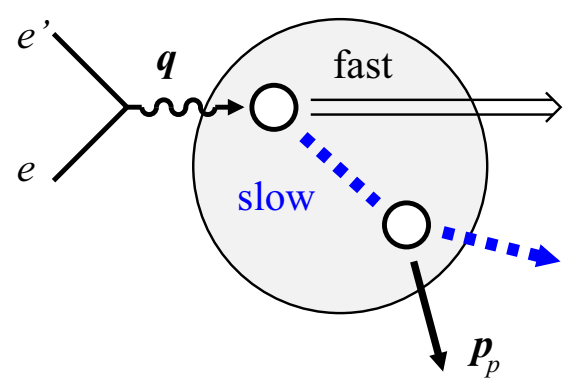

FIG. 1. Physical picture of FSI in electron-deuteron DIS with proton tagging, $e+d \rightarrow e^{\prime}+p\left(p_{p}\right)+X$, in the deuteron rest frame. A slow hadron in the final state produced by DIS on the active neutron scatters from the spectator proton, changing its momentum compared to the IA. The fast component of the DIS final state does not interact strongly with the spectator.

region explored with a medium-energy EIC. We use methods of LF quantum mechanics to separate nuclear and nucleonic structure in the high-energy process and enable dynamical calculations of the deuteron structure elements. We derive the tagged structure functions in the IA and study their symmetries and analytic properties in the recoil momentum. We then develop a dynamical model of nuclear FSI at "intermediate" $x$, defined as the region between the extreme valence quark regime at $x \gtrsim 0.5$ and the coherent regime at $x \ll 0.1$ (the role of coherent phenomena in FSI at $x \ll 0.1$ will be considered in a separate study [22]). This intermediate region is of prime interest for the study of sea quarks and gluons and their nuclear modifications. We use our model to estimate the magnitude and kinematic dependence of the FSI effects, demonstrate their analytic properties, and study the implications for neutron structure extraction through on-shell extrapolation.

Our treatment is based on a definite physical picture of FSI at intermediate $x$. The DIS process on the nucleon with momentum transfers $|\boldsymbol{q}| \gg 1 \mathrm{GeV}$ (in the deuteron rest frame) produces a broad spectrum of hadrons, ranging in momenta from $\left|\boldsymbol{p}_{h}\right| \sim|\boldsymbol{q}|$ to $\left|\boldsymbol{p}_{h}\right| \lesssim 1 \mathrm{GeV}$. The "fast" part of the nucleon DIS final state does not interact strongly with the spectator nucleons in the nucleus. This assertion is supported by empirical and theoretical arguments. Nuclear DIS data show that fast hadrons with $\left|\boldsymbol{p}_{h}\right| \gg 1 \mathrm{GeV}$ are not substantially attenuated in nuclei [23-28]; the soft neutron spectra produced by nuclear breakup in DIS likewise indicate the absence of strong FSIs [26,29]. Theoretical estimates of the hadron formation time show that such fast hadrons form mainly outside of the nucleus and cannot interact with hadronic cross sections; see Sec. 5.10 of Ref. [5] for an overview. The dominant FSI in tagged DIS on the deuteron therefore comes from "slow" hadrons with $\left|\boldsymbol{p}_{h}\right| \lesssim 1 \mathrm{GeV}$ in the nucleon DIS final state. Such hadrons are formed inside the nucleus and can interact with the spectator with hadronic cross sections (see Fig. 1). In the terminology of DIS, the "fast" and "slow" parts of the final state of DIS on the nucleon correspond to the current and target fragmentation regions (see Sec. V). We note that the physical picture of FSIs proposed here is consistent with the general QCD factorization theorem for target fragmentation in DIS, which is a rigorous asymptotic result and holds irrespective of the type of target (nucleon or nucleus) [30,31]. FSI of the "fast" DIS hadrons with the nuclear remnant would amount to a violation of factorization for the nuclear target in the asymptotic regime. In contrast, FSI of the "slow" DIS hadrons with the nuclear remnant represent a particular soft-interaction contribution to the nuclear fracture function that is allowed by the factorization theorem.

We express this physical picture of FSI in a schematic model. We calculate the tagged DIS cross section on the deuteron using LF quantum mechanics, including the IA and FSI amplitudes. We use empirical hadron distributions, measured in ep/ed DIS, to describe the slow part of the hadronic final state produced on the nucleon in the deuteron. The interactions of the slow hadrons with the spectator are treated as on-shell scattering with an effective cross section. Off-shell effects can be absorbed into the effective cross section and the slow hadron distribution; they are physically indistinguishable from effects of the finite hadron formation time and can consistently be accounted for in this way. This model amounts to a minimal description of FSIs based on the space-time evolution of the DIS process and empirical hadron distributions.

In the present study we use the apparatus of LF quantum mechanics to describe the initial-state nuclear structure and final-state interactions in tagged DIS. High-energy processes such as DIS effectively probe a strongly interacting system at fixed LF time $x^{+}=x^{0}+x^{3}$, along the direction defined by the reaction axis. In LF quantization one follows the time evolution of the system in $x^{+}$and describes its structure by wave functions and densities at $x^{+}=$const [32-36]. The scheme is unique in that it permits a composite description in which effects of the off-shellness of the constituents remain finite as the scattering energy becomes large [11]. It makes possible a composite description of nuclear structure in DIS in terms of nucleon degrees of freedom, which exhibits a close correspondence with nonrelativistic nuclear structure ( $N N$ interactions, wave functions), satisfies sum rules (baryon number, LF momentum), and enables a smooth matching with nucleon structure (parton picture, QCD degrees of freedom) $[1,11]$. It is important to understand that the structure thus described is "low-energy" nuclear structure, governed by interactions and degrees of freedom on the nuclear scale; it is only presented in a way that is appropriate for the initial state of high-energy processes. The application of LF quantum mechanics to nuclear high-energy processes is described in detail in Refs. [1,11]; the elements used in the present calculation are summarized below.

Conservation of baryon number and LF momentum is an important consideration in describing nuclear DIS. The LF IA for the inclusive DIS structure functions correctly implements the baryon number and momentum sum rules for the deuteron, i.e., the baryon numbers of the $p$ and $n$ add up to the total baryon number of the deuteron when integrating over all configurations in the wave function, and the LF "plus" momenta of the $p$ and $n$ add up to the total momentum of the deuteron [11]. It means that at this level there are no non-nucleonic degrees of freedom and ensures that, when the nucleons are resolved into partons, the partonic sum rules for the deuteron are satisfied. In the tagged DIS structure 
functions one recovers these sum rules when integrating over the spectator recoil momentum. FSIs in tagged DIS may distort the recoil momentum distribution but should not modify the sum rules for the recoil momentum-integrated structure functions. In our picture this can be accomplished by modeling the slow hadron-nucleon rescattering process as elastic scattering (no additional hadrons are produced) and implementing unitarity of the rescattering amplitude. In this sense our model of FSIs in tagged DIS preserves the baryon number and momentum sum rules and is consistent with the standard LF treatment of the inclusive DIS structure functions.

The plan of the article is as follows. In Sec. II we present the kinematic variables and invariant structure functions in tagged DIS, introduce the collinear frame used in the LF description, and discuss the recoil momentum variables. In Sec. III we summarize the elements of LF quantum mechanics used in our calculations - the single-nucleon states, the deuteron LF wave function, its rotationally symmetric representation, and the treatment of spin degrees of freedom. In Sec. IV we calculate the tagged DIS cross section in the IA and study its properties. We discuss the LF current components, compute the IA current and structure functions, introduce the LF spectral function, and discuss the nonrelativistic limit and the analytic properties in $t$. In Sec. $\mathrm{V}$ we discuss the slow hadron distribution in DIS on the nucleon-the kinematic variables, structure functions, and the features of empirical distributions. In Sec. VI we calculate the FSI effects in tagged DIS in LF quantum mechanics and study their properties. The FSI effects are expressed in terms of a distorted spectral function. We include the spin degrees of freedom and formulate a nonrelativistic approximation for evaluating the rescattering integral. We demonstrate the positivity of the cross section, investigate the recoil momentum dependence of the FSI effects, and discuss the analytic properties of the distorted spectral function. We also comment on the role of unitarity of the rescattering process and the implementation of sum rules for the LF spectral function with FSI. In Sec. VII we discuss the strategy for neutron structure measurements in tagged DIS with EIC. A summary and outlook are given in Sec. VIII.

Technical material needed to reproduce the calculations is summarized in the appendixes. Appendix A describes the nonrelativistic deuteron wave function used in constructing the deuteron LF wave function and evaluating the IA and FSI expressions. Appendix B contains the projection formulas for extracting the tagged structure functions from the deuteron tensor in the IA and with FSIs. Appendix C summarizes our parametrization of the nucleon-nucleon cross section used in the numerical estimates of FSI effects. Appendix D describes the evaluation of the rescattering phase space integral in the FSI amplitude.

The physical picture of FSIs in tagged DIS proposed here is specific to the region of intermediate $x$ as defined above, and our model should be used in this context only. In tagged DIS in the limit $x \rightarrow 1$, the minimal rest-frame momentum of the "slow" hadrons produced on the nucleon becomes large, as their LF momentum fractions are bounded by $1-x$, and the interactions are suppressed by the hadron formation time (see Fig. 1). The framework developed in the present work could be extended to this region when supplied with empirical informa- tion about the formation time effects. (The work of Refs. [3739] considers tagged DIS at large $x$ in a subasymptotic domain of fixed energy and momentum transfer, where the limit $x \rightarrow 1$ corresponds to a small inelasticity $W^{2}-M_{N}^{2}$, and the DIS final state is modeled as a superposition of baryonic resonances; this formulation is different from the asymptotic one presented here and not appropriate for collider energies.) In tagged DIS at small $x(\ll 0.1)$, diffractive scattering on the nucleon becomes significant and gives rise to a new mechanism of FSI. In diffractive scattering the nucleon appears intact in the final state and recoils with a momentum of $\sim$ few $100 \mathrm{MeV}$. Because the diffractive nucleon retains its quantum numbers ("vacuum exchange") and emerges with a small recoil momentum, there is a significant amplitude for the final-state $p n$ system to revert back to a deuteron bound state. In tagged DIS in this kinematics the outgoing $p n$ scattering state must be properly orthogonalized to the deuteron bound state, which results in significant distortion. This new mechanism of FSI in tagged DIS on the deuteron at small $x$ is closely related to shadowing in inclusive DIS and will be discussed elsewhere [22].

In the present work we consider unpolarized electrondeuteron DIS and calculate the FSI effects in the tagged cross section integrated over the azimuthal angle of the recoil momentum, as relevant for the extraction of the neutron structure functions $F_{2 n}$ and $F_{L n}$. The extension to polarized electron-deuteron DIS with spectator tagging and azimuthal angle-dependent response functions will be left to a future study, as the number of structures in the cross section becomes very considerable [40]. A proper treatment of FSIs in the polarized deuteron would require also empirical information on the spin dependence of the slow DIS hadron distributions, which is not available at present. We note that the schematic model of FSIs proposed here could be applied also to the time-reversal-odd ( $T$-odd) response functions in tagged DIS, which are zero in the IA and can be used for sensitive tests of the FSI dynamics.

The internal spin structure of the deuteron plays an important role in tagged DIS. The nonrelativistic deuteron wave function has $S$ - and $D$-wave components, in which the nucleon spins are effectively polarized along and opposite to the deuteron spin; the $D$ wave dominates the momentum density at nucleon momenta $\left|\boldsymbol{p}_{N}\right| \gtrsim 300 \mathrm{MeV}$. This structure can be incorporated in the LF description using the correspondence between the nonrelativistic and LF wave functions [41]. In the LF formulation the nucleon spin states are chosen as LF helicity states, which are obtained from the rest-frame spin states by a sequence of LF boosts and differ from ordinary helicity states by a spin rotation [33-36]. In this work we include both the $S$ - and the $D$-wave component in the LF calculation of the tagged DIS cross section in the IA and the FSI correction. Our treatment is simplified by two circumstances: (a) There is little theoretical or experimental evidence for polarization of the slow hadrons emerging from the DIS process. We therefore treat these hadrons as unpolarized and neglect the dependence of the rescattering process on the spectator spin. This avoids the need of dealing with spin rotation effects and results in the absence of $S$ - $D$-wave mixing in the linear FSI correction (interference of IA and FSI amplitudes). (b) Our interest here is in the tagged DIS cross section at small 
recoil momenta $\left|\boldsymbol{p}_{p}\right|<200 \mathrm{MeV}$, where the IA amplitude is dominated by the $S$ wave. The main FSI correction results from the $S$-wave IA interfering with the $S$-wave FSI amplitude, in which the rescattering integral is dominated by proton momenta $<300 \mathrm{MeV}$. The rescattering integral with the $D$ wave, which involves larger momenta, appears in the final result only together with the $D$-wave IA amplitude, which is itself small. We therefore do not need an accurate treatment of the high-momentum contributions in the $D$-wave rescattering integral. We use a nonrelativistic approximation to the LF wave function, accurate up to terms $O\left(p^{2} / M_{N}^{2}\right)$, to calculate both $S$ and $D$-wave contributions to the IA and the FSI amplitudes. Together, these assumptions permit an efficient description of the spin degrees of freedom that is adequate for the purpose of our study.

In order to simplify the presentation, we initially suppress the spin degrees of freedom and derive the LF expressions for the IA and FSI amplitudes for a bound state of spinless nucleons. This allows us to focus on the aspects essential to the LF formulation (flux factors) without the complications resulting from spin. We then include the spin degrees of freedom in the LF expressions by way of generalization. Finally, we derive the nonrelativistic approximation to the LF quantities of interest here (IA spectral function, FSI correction) and use it for numerical evaluation.

FSI effects in DIS from nuclei and their kinematic dependence were studied in Refs. [42-45] using a detailed microscopic model of hadron production in DIS (string breaking, gluon radiation). In contrast to these studies we use a simple generic description of hadron production and consider specifically the region of intermediate $x$. FSI effects have also been studied extensively in quasielastic scattering from nuclei, including deuteron electrodisintegration $e+d \rightarrow e^{\prime}+p+n$ [46]; see Ref. [47] for a review. There is an interesting formal analogy between FSIs in quasielastic deuteron breakup at $\sim 1-2 \mathrm{GeV}$ incident momenta and our picture of slow-hadron rescattering in DIS, and one can establish the correspondence between the formulas.

\section{TAGGED DIS KINEMATICS}

\section{A. Kinematic variables}

We begin by summarizing the kinematic variables and cross section formulas for inclusive electron scattering on the deuteron with an identified nucleon in the final state ("tagged DIS"). The kinematic factors are given in their exact form (no simplifications are made using the DIS limit) and expressed in terms of relativistic invariants, as suitable for collider experiments. The cross section formulas given in this section are general and make no assumption regarding composite nuclear structure; particular results based on such approximations will be presented in Secs. IV and VI. To be specific we consider the case that the identified nucleon is a proton; equivalent formulas can be written for the case of an identified neutron. Thus, we consider the scattering process (see Fig. 2)

$$
e\left(p_{e}\right)+d\left(p_{d}\right) \rightarrow e^{\prime}\left(p_{e^{\prime}}\right)+p\left(p_{p}\right)+X,
$$

where $X$ denotes an unresolved hadronic final state. Here $p_{e}$ and $p_{e^{\prime}}$ are the four-momenta of the initial and final electron,

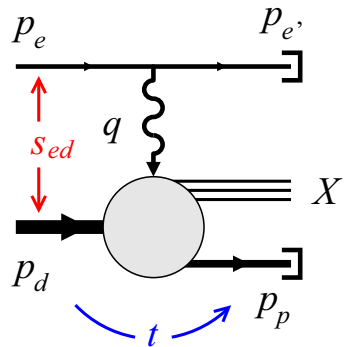

FIG. 2. Inclusive electron scattering from the deuteron with an identified proton in the deuteron fragmentation region, $e+d \rightarrow e^{\prime}+$ $p+X$ ("tagged DIS").

$p_{d}$ is the four-momentum of the deuteron, and $p_{p}$ is the four-momentum of the identified proton. The four-momentum transfer to the nuclear system, calculated from the initial and final electron four-momenta, is

$$
q \equiv p_{e}-p_{e^{\prime}}, \quad Q^{2} \equiv-q^{2}>0 .
$$

Invariants formed from the electron and deuteron four-momenta are

$$
\begin{aligned}
s_{e d} & =\left(p_{e}+p_{d}\right)^{2}, \\
W_{d}^{2} & =\left(q+p_{d}\right)^{2},
\end{aligned}
$$

which describe, respectively, the electron-deuteron and the virtual photon-deuteron squared CM energies. Useful scaling variables are

$$
\begin{aligned}
& x_{d} \equiv \frac{-q^{2}}{2\left(p_{d} q\right)}=\frac{Q^{2}}{W_{d}^{2}-M_{d}^{2}+Q^{2}}, \\
& y \equiv \frac{\left(p_{d} q\right)}{\left(p_{d} p_{e}\right)}=\frac{Q^{2}}{x_{d}\left(s_{e d}-M_{d}^{2}\right)} .
\end{aligned}
$$

The variable $x_{d}$ is the Bjorken variable for the nuclear target $\left(0<x_{d}<1\right)$ and will be used in the kinematic formulas for the cross section, to facilitate comparison with the standard expressions for electron-proton scattering. In the description of composite deuteron structure we shall use the alternative variable

$$
x \equiv 2 x_{d} \quad(0<x<2),
$$

which corresponds to the effective Bjorken variable for scattering from a nucleon in the unbound nucleus (deuteron). The variable $y$ describes the electron's fractional energy loss (or inelasticity) in the deuteron rest frame. The invariants and scaling variables formed with the recoil nucleon momentum $p_{p}$ will be presented in Sec. IID below.

\section{B. Cross section and structure functions}

The invariant amplitude for the electroproduction of a final state $p+X$, including the detected proton $p$ and a specified set of hadrons $X$, is in leading order of the electromagnetic coupling given by

$$
\begin{aligned}
& \mathcal{M}\left[e d \rightarrow e^{\prime} p X\right] \\
& =\frac{e^{2}}{q^{2}}\left\langle e^{\prime}, \boldsymbol{p}_{e^{\prime}}\left|\hat{J}^{\mu}(0)\right| e, \boldsymbol{p}_{e}\right\rangle\left\langle p, \boldsymbol{p}_{p} ; X\left|\hat{J}^{\mu}(0)\right| d, \boldsymbol{p}_{d}\right\rangle,
\end{aligned}
$$


where $e$ is the elementary charge, and the brackets denote the transition matrix elements of the electromagnetic fourvector current $\hat{J}^{\mu}(0)$ between the initial and final electron and nuclear/hadronic states. All particle states (electron, deuteron, nucleon) are normalized according to the relativistic convention

$$
\left\langle e, \boldsymbol{p}_{e 2} \mid e, \boldsymbol{p}_{e 1}\right\rangle=(2 \pi)^{3} 2 E_{e}\left(\boldsymbol{p}_{e 1}\right) \delta^{(3)}\left(\boldsymbol{p}_{e 2}-\boldsymbol{p}_{e 1}\right), \text { etc. }
$$

The spin quantum numbers of the states are suppressed for brevity and will be specified below. The differential cross section for production of the specified hadronic state is [48]

$$
\begin{aligned}
d \sigma\left[e d \rightarrow e^{\prime} p X\right]= & (2 \pi)^{4} \delta^{(4)}\left(p_{e}+p_{d}-p_{e^{\prime}}-p_{p}-p_{h}\right) \\
& \times \frac{|\mathcal{M}|^{2}}{4 I} d \Gamma_{e^{\prime}} d \Gamma_{p} d \Gamma_{X} .
\end{aligned}
$$

The invariant incident particle current is defined as (we neglect the electron mass)

$$
I \equiv \sqrt{\left(p_{e} p_{d}\right)^{2}} \equiv \frac{1}{2}\left(s_{e d}-M_{d}^{2}\right) .
$$

The invariant phase space elements of the scattered electron and the identified proton are

$$
\begin{aligned}
& d \Gamma_{e^{\prime}} \equiv \frac{d^{3} p_{e^{\prime}}}{(2 \pi)^{3} 2 E_{e^{\prime}}}, \quad E_{e^{\prime}}=\left|\boldsymbol{p}_{e^{\prime}}\right|, \\
& d \Gamma_{p} \equiv \frac{d^{3} p_{p}}{(2 \pi)^{3} 2 E_{p}}, \quad E_{p}=\sqrt{\left|\boldsymbol{p}_{p}\right|^{2}+M_{N}^{2}} .
\end{aligned}
$$

The phase space element of the multi-hadron state $X$ can be defined analogously in terms of the hadron momenta, but its explicit form is not needed in the following. The cross section for tagged inclusive scattering is then given by integrating Eq. (2.10) over the phase space of the unidentified hadron state $X$ and summing over all such states (we denote both operations together symbolically by $\sum_{X}$ ),

$$
\begin{aligned}
d \sigma\left[e d \rightarrow e^{\prime} p X\right]= & \sum_{X}(2 \pi)^{4} \delta^{(4)}\left(p_{e}+p_{d}-p_{e^{\prime}}-p_{p}-p_{X}\right) \\
& \times \frac{|\mathcal{M}|^{2}}{4 I} d \Gamma_{e^{\prime}} d \Gamma_{p} \\
= & \frac{4 \pi e^{4}}{4 I\left(-q^{2}\right)^{2}} w_{\mu \nu} W_{d}^{\mu \nu} d \Gamma_{e^{\prime}} d \Gamma_{p} .
\end{aligned}
$$

The leptonic tensor is defined as

$$
\begin{aligned}
w^{\mu \nu} & \equiv w^{\mu \nu}\left(p_{e^{\prime}}, p_{e}\right) \\
& =\left\langle e^{\prime}, \boldsymbol{p}_{e^{\prime}}\left|\hat{J}^{\mu}(0)\right| e, \boldsymbol{p}_{e}\right\rangle^{*}\left\langle e^{\prime}, \boldsymbol{p}_{e^{\prime}}\left|\hat{J}^{v}(0)\right| e, \boldsymbol{p}_{e}\right\rangle .
\end{aligned}
$$

In the case of scattering of an unpolarized electron beam (average over initial helicities) and unspecified polarization of the final electron (sum over final helicities) its explicit form is

$$
w^{\mu \nu}=4 p_{e}^{\mu} p_{e}^{\nu}+q^{2} g^{\mu \nu}+\text { terms } \propto q^{\mu}, q^{\nu} .
$$

The deuteron tensor is defined as (using $q=p_{e}-p_{e^{\prime}}$ )

$$
\begin{aligned}
W_{d}^{\mu \nu} \equiv & W_{d}^{\mu \nu}\left(p_{d}, q, p_{p}\right) \\
= & (4 \pi)^{-1} \sum_{X}(2 \pi)^{4} \delta^{(4)}\left(q+p_{d}-p_{p}-p_{X}\right) \\
& \times\left\langle p X\left|\hat{J}^{\mu}(0)\right| d\right\rangle^{*}\left\langle p X\left|\hat{J}^{\nu}(0)\right| d\right\rangle
\end{aligned}
$$

$$
\begin{aligned}
= & (4 \pi)^{-1} \sum_{X}(2 \pi)^{4} \delta^{(4)}\left(q+p_{d}-p_{p}-p_{X}\right) \\
& \times\left\langle d\left|\hat{J}^{\mu \dagger}(0)\right| p X\right\rangle\left\langle p X\left|\hat{J}^{\nu}(0)\right| d\right\rangle,
\end{aligned}
$$

in which $J^{\mu \dagger}=J^{\mu}$ (hermiticity of the electromagnetic current operator). We consider the case of scattering from an unpolarized deuteron (average over deuteron spin) and unspecified polarization of the final proton (sum over final proton spin); the spin sums will be shown explicitly in the calculations below. The tensor obeys the transversality conditions $q_{\mu} W_{d}^{\mu \nu}=0$ and $W_{d}^{\mu v} q_{v}=0$ and can be expanded in tensors constructed from the four-vectors $p_{d}, q$ and $p_{p}$, and the invariant tensor $g^{\mu \nu}$. It is convenient to introduce the auxiliary four-vectors

$$
\begin{aligned}
L^{\mu} & \equiv p_{d}^{\mu}-\frac{\left(p_{d} q\right) q^{\mu}}{q^{2}}, \quad(q L)=0, \quad L^{2}>0, \\
T^{\mu} & \equiv p_{p}^{\mu}-\frac{\left(p_{p} q\right) q^{\mu}}{q^{2}}-\frac{\left(p_{p} L\right) L^{\mu}}{L^{2}} \\
(q T) & =0, \quad(L T)=0, \quad T^{2}<0 .
\end{aligned}
$$

Their particular meaning in a frame where $p_{d}$ and $q$ are collinear is explained in Sec. II C. We decompose the deuteron tensor as

$$
\begin{aligned}
W_{d}^{\mu \nu}= & \left(g^{\mu \nu}-\frac{q^{\mu} q^{\nu}}{q^{2}}\right) \frac{F_{L d}}{2}+\left(\frac{L^{\mu} L^{\nu}}{L^{2}}+\frac{q^{\mu} q^{\nu}}{q^{2}}-g^{\mu \nu}\right) \frac{F_{T d}}{2} \\
& +\frac{T^{\mu} L^{\nu}+L^{\mu} T^{\nu}}{\sqrt{-T^{2}} \sqrt{L^{2}}} \frac{F_{L T, d}}{2} \\
& +\left(g^{\mu \nu}-\frac{L^{\mu} L^{\nu}}{L^{2}}-\frac{q^{\mu} q^{\nu}}{q^{2}}-\frac{2 T^{\mu} T^{\nu}}{T^{2}}\right) \frac{F_{T T, d}}{2} .
\end{aligned}
$$

Here $F_{L d}, F_{T d}, F_{L T, d}$, and $F_{T T, d}$ are invariant structure functions, depending on the kinematic invariants formed from the vectors $q, p_{d}$ and $p_{p}$. The first and second tensor structures in Eq. (2.22) do not involve the identified proton momentum $p_{p}$ and are present also in untagged (fully inclusive) scattering. Our definition of the longitudinal and transverse structure functions, $F_{L d}$ and $F_{T d}$, is identical to that of Ref. [49]. Their relation to the conventional functions $F_{1 d}$ and $F_{2 d}$ is

$$
\begin{aligned}
F_{T d} & =\frac{\left(-q^{2}\right) L^{2}}{\left(p_{d} q\right)^{2}} \frac{F_{2 d}}{x_{d}}=\left(1+\frac{4 x_{d}^{2} M_{d}^{2}}{Q^{2}}\right) \frac{F_{2 d}}{x_{d}} \\
F_{L d} & =\frac{\left(-q^{2}\right) L^{2}}{\left(p_{d} q\right)^{2}} \frac{F_{2 d}}{x_{d}}-2 F_{1 d} \\
& =\frac{1}{x_{d}}\left[\left(1+\frac{4 x_{d}^{2} M_{d}^{2}}{Q^{2}}\right) F_{2 d}-2 x_{d} F_{1 d}\right], \\
F_{T d}-F_{L d} & =2 F_{1 d}
\end{aligned}
$$

The third and fourth tensor structures in Eq. (2.22) vanish when averaging over the orientation of the vector $T$ in the plane orthogonal to $L$ and $q$ and are present only for fixed momentum $p_{p}$. 
The contraction of the leptonic and deuteron tensors can be expressed in terms of the parameter

$$
\epsilon \equiv \frac{w_{\mu \nu} \frac{L^{\mu} L^{v}}{L^{2}}}{w_{\mu \nu}\left(\frac{L^{\mu} L^{v}}{L^{2}}+\frac{q^{\mu} q^{v}}{q^{2}}-g^{\mu \nu}\right)}=\frac{1-y-\frac{x_{d}^{2} y^{2} M_{d}^{2}}{Q^{2}}}{1-y+y^{2} / 2+\frac{x_{d}^{2} y^{2} M_{d}^{2}}{Q^{2}}},
$$

which can be interpreted as the ratio of the probabilities of longitudinal and transverse polarization of the virtual photon. To express the contractions of the $p_{p}$-dependent tensor structures in invariant form we expand the initial electron momentum as

$$
\begin{gathered}
p_{e}=\frac{\left(p_{e} L\right) L}{L^{2}}+\frac{\left(p_{e} q\right) q}{q^{2}}+\Delta, \quad(\Delta L)=0, \quad(\Delta q)=0, \\
\left(p_{e} T\right)=(\Delta T)=\sqrt{-\Delta^{2}} \sqrt{-T^{2}} \cos \phi_{p} .
\end{gathered}
$$

The particular meaning of the angle $\phi_{p}$ in a collinear frame is described in Sec. IIC. We obtain

$$
\begin{aligned}
w_{\mu \nu} W^{\mu \nu}= & \frac{Q^{2}}{1-\epsilon}\left[\frac{1+\epsilon}{1-\epsilon} \frac{y^{2}}{(2-y)^{2}} F_{T d}-(1-\epsilon) F_{L d}\right. \\
& \left.+\sqrt{2 \epsilon(1+\epsilon)} \cos \phi_{p} F_{L T, d}+\epsilon \cos \left(2 \phi_{p}\right) F_{T T, d}\right] \\
= & \frac{Q^{2}}{1-\epsilon}\left[\frac{F_{2 d}}{x_{d}}-(1-\epsilon) F_{L d}+\cdots\right]
\end{aligned}
$$

The scattered electron phase space element can easily be expressed in terms of the invariants $x_{d}$ and $Q^{2}$ and the azimuthal angle around the incident electron momentum direction $\phi_{e^{\prime}}$. Altogether, the differential cross section for tagged inclusive scattering with unpolarized beams and recoil nucleon, Eq. (2.15), becomes

$$
\begin{aligned}
& d \sigma\left[e d \rightarrow e^{\prime} p X\right]=\frac{2 \pi \alpha_{\mathrm{em}}^{2} y^{2}}{Q^{4}(1-\epsilon)} d x_{d} d Q^{2} \frac{d \phi_{e^{\prime}}}{2 \pi} \\
& \quad \times\left[\frac{F_{2 d}}{x_{d}}-(1-\epsilon) F_{L d}+\sqrt{2 \epsilon(1+\epsilon)} \cos \phi_{p} F_{L T, d}+\epsilon \cos \left(2 \phi_{p}\right) F_{T T, d}\right] d \Gamma_{p},
\end{aligned}
$$

where $\alpha_{\mathrm{em}} \equiv e^{2} /(4 \pi) \approx 1 / 137$ is the fine structure constant. The last two terms in the bracket drop out when the cross section is integrated over the recoil azimuthal angle $\phi_{p}$. Specific forms of the recoil momentum phase space element are described in Sec. IID.

\section{Collinear frames}

In the theoretical description of tagged DIS we consider the process Eq. (2.1) in a frame where the deuteron momentum $\boldsymbol{p}_{d}$ and the momentum transfer $\boldsymbol{q}$ are collinear and define the $z$-axis of the coordinate system. This condition does not specify a unique frame, but rather a class of frames that are related by boosts along the $z$-axis ("collinear frames"). We specify the four-momenta in this frame by their LF components

$$
p^{ \pm} \equiv p^{0} \pm p^{z}, \quad \boldsymbol{p}_{T} \equiv\left(p^{x}, p^{y}\right)
$$

The LF components of $p_{d}$ and $q$ in the collinear frame are

$$
\left.\begin{array}{lll}
p_{d}^{+}>0 \text { (arbitrary), } & p_{d}^{-}=\frac{M_{d}^{2}}{p_{d}^{+}}, & \boldsymbol{p}_{d T}=0 \\
q^{+}=-\xi_{d} p_{d}^{+}, & q^{-}=\frac{q^{2}}{q^{+}}=\frac{Q^{2}}{\xi_{d} p_{d}^{+}}, & \boldsymbol{q}_{T}=0
\end{array}\right\}
$$

The parameter $\xi_{d}$ is fixed by the condition

$$
2 p_{d} q=p_{d}^{+} q^{-}+p_{d}^{-} q^{+}=\frac{Q^{2}}{x_{d}},
$$

the solution of which is

$$
\xi_{d}=\frac{2 x_{d}}{1 \pm \sqrt{1+4 M_{d}^{2} x_{d}^{2} / Q^{2}}} \quad(\text { choose }+) .
$$

We select the solution with the plus sign, which has the property that in the scaling limit $Q^{2} \gg M_{d}^{2}$

$$
\xi_{d}=x_{d}+O\left(M_{d}^{2} / Q^{2}\right)
$$

With this choice the momentum transfer vector $\boldsymbol{q}$ points in the negative $z$ direction (see Fig. 3),

$$
2 q^{z}=q^{+}-q^{-}=-\xi_{d} p_{d}^{+}-\frac{Q^{2}}{\xi_{d} p_{d}^{+}}<0 .
$$

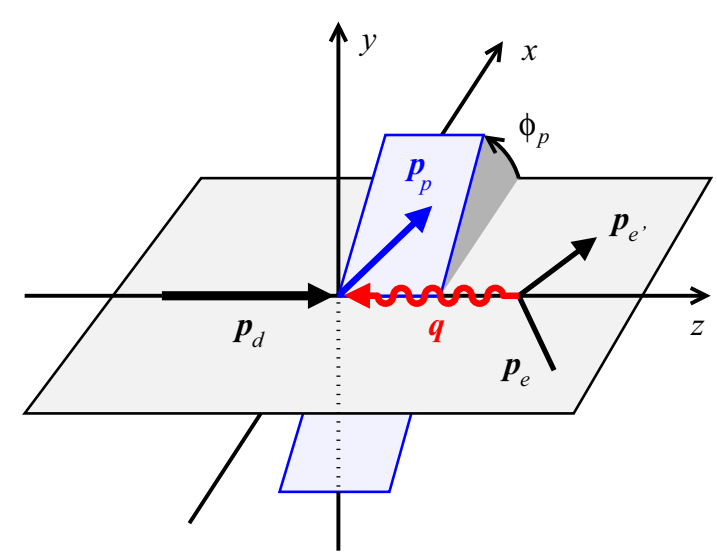

FIG. 3. Tagged DIS in the collinear frame, Eq. (2.33). The deuteron momentum $\boldsymbol{p}_{d}$ and the vector $\boldsymbol{q}$ are collinear and define the $z$ axis, with $\boldsymbol{q}$ pointing in the negative $z$ direction. The initial and final electron momenta lie in the $x z$ plane and have positive $x$ component. $\phi_{p}$ is the angle of the transverse $(x y)$ component of the recoil momentum, measured relative to the positive $x$ axis. 
The LF components of the longitudinal auxiliary fourvectors $L$ and $T$, Eqs. (2.20) and (2.21), are obtained as

$$
\left.\begin{array}{lll}
L^{+}=\left(1-\frac{\xi_{d}}{2 x_{d}}\right) p_{d}^{+}, & L^{-}=\left(M_{d}^{2}+\frac{Q^{2}}{\xi_{d}}\right) \frac{1}{p_{d}^{+},}, & \boldsymbol{L}_{T}=0 \\
T^{+}=0, & \boldsymbol{T}_{T}=\boldsymbol{p}_{p T}
\end{array}\right\} .
$$

The vector $L$ has only collinear components, while $T$ has only transverse components and coincides with the recoil hadron transverse momentum in the collinear frame. Because the momentum transfer $\boldsymbol{q}$ is along the (negative) $z$ direction, the initial and final electron momenta have the same transverse components,

$$
\boldsymbol{p}_{e T}=\boldsymbol{p}_{e^{\prime} T} \quad \text { (collinear frame), }
$$

such that they define a plane together with the $z$ axis (electron scattering plane). The recoil angle $\phi_{p}$, defined in terms of invariants in Eq. (2.28), then is the azimuthal angle of $\boldsymbol{p}_{p}$, measured relative to the electron scattering plane. It is conventional to choose the electron transverse momenta in the $x$ direction, such that the electron scattering plane is the $x z$ plane. In this case the angle $\phi_{p}$ becomes the conventional azimuthal angle of $\boldsymbol{p}_{p}$ in the $x y$ plane, $\cos \phi_{p}=p_{p}^{x} /\left|\boldsymbol{p}_{p T}\right|$ (see Fig. 3).

The deuteron plus momentum $p_{d}^{+}>0$ in the above formulas remains arbitrary and defines a particular member of the class of collinear frames. Longitudinal boosts (along the $z$ axis) can be performed simply by changing the value of $p_{d}^{+}$in the above formulas. Note that the class of collinear frames contains several special cases of interest: (a) the target rest frame, $p_{d}^{+}=M_{d}$; (b) the Breit frame, $p_{d}^{+}=\sqrt{Q^{2}} / \xi_{d}$, in which $q^{0}=\left(q^{+}+q^{-}\right) / 2=0$; (c) the CM frame of the virtual photon and the deuteron, $p_{d}^{+}=\sqrt{Q^{2}+\xi_{d} M_{d}^{2}} / \sqrt{\xi_{d}\left(1-\xi_{d}\right)}$, in which $q^{z}=\left(q^{+}-q^{-}\right) / 2=-p_{d}^{z}=-\left(p_{d}^{+}-p_{d}^{-}\right) / 2$ and thus $\boldsymbol{q}=-\boldsymbol{p}_{d}$. For reference we note that the collinear frames used here are equivalent to the covariant formulation of the collinear expansion in terms of lightlike vectors of Ref. [49].

\section{Recoil momentum variables}

The tagged structure functions of the deuteron in Eq. (2.22) depend on the usual DIS variables (e.g., $W_{d}$ and $Q^{2}$ ) as well as the recoil nucleon momentum. The latter dependence involves two independent variables formed from $p_{p}$, related to the two invariants $\left(p_{p} p_{d}\right)$ and $\left(p_{p} q\right)$; the dependence on the third invariant $\left(p_{p} p_{e}\right)$ is encoded in the explicit $\phi_{p}$ dependence of the cross section. Here we describe several physically interesting choices of recoil momentum variables that are used in the subsequent calculations. We present their relation to the rest-frame recoil momentum, their kinematic limits, and the corresponding phase space elements. We assume isospin symmetry and define the nucleon mass as the average of the proton and neutron masses

$$
M_{N}=\left(M_{p}+M_{n}\right) / 2=0.9389 \mathrm{GeV} .
$$

The deuteron binding energy and mass are taken at their exact values

$$
\begin{aligned}
\epsilon_{d} & =2.2 \mathrm{MeV} \\
M_{d} & =M_{p}+M_{n}-\epsilon_{d}=2 M_{N}-\epsilon_{d}=1.8756 \mathrm{GeV} .
\end{aligned}
$$

Note that the relation between the deuteron binding energy and mass is not affected when replacing the proton and neutron masses by their average.

In a collinear frame defined by Eqs. (2.33) the tagged structure functions can be regarded as functions of the LF plus momentum fraction of the recoil proton and the transverse momentum modulus of the recoil momentum,

$$
\begin{aligned}
\alpha_{p} & \equiv \frac{2 p_{p}^{+}}{p_{d}^{+}}=\frac{2\left(p_{p}^{0}+p_{p}^{z}\right)}{p_{d}^{0}+p_{d}^{z}}, \\
\left|\boldsymbol{p}_{p T}\right| & \equiv \sqrt{\left(p_{p}^{x}\right)^{2}+\left(p_{p}^{y}\right)^{2}} .
\end{aligned}
$$

The definition of $\alpha_{p}$ in Eq. (2.42), as the fraction relative to $p_{d}^{+} / 2$, is natural and leads to simple expressions in the nuclear structure calculations below. The kinematic limits of $\alpha_{p}$ are dictated by LF plus momentum conservation in the scattering process and given by

$$
\alpha_{p} / 2<1-\xi_{d} .
$$

The invariant phase space element in terms of $\alpha_{p}$ and $\left|\boldsymbol{p}_{p T}\right|$ takes the form

$$
\frac{d^{3} p_{p}}{E_{p}}=\frac{d \alpha_{p}}{\alpha_{p}} d^{2} p_{p T} .
$$

An important variable is the invariant momentum transfer between the initial-state deuteron and the final-state nucleon (see Fig. 2),

$$
t \equiv\left(p_{p}-p_{d}\right)^{2},
$$

or the reduced variable

$$
t^{\prime} \equiv t-M_{N}^{2}
$$

The theoretical analysis of tagged DIS, Eq. (2.1), relies essentially on the analytic properties of the cross section in $t^{\prime}$; see Secs. IV and VI below. The invariant $t^{\prime}$ is related in a simple manner to the energy of the recoiling nucleon in the deuteron rest frame (we use "RF" to denote rest-frame energy and momentum),

$$
\begin{aligned}
t^{\prime} & =M_{d}^{2}-2 M_{d} E_{p}(\mathrm{RF}), \\
{\left[E_{p}(\mathrm{RF})\right.} & \left.\equiv \sqrt{\left|\boldsymbol{p}_{p}(\mathrm{RF})\right|^{2}+M_{N}^{2}}\right] .
\end{aligned}
$$

The kinematic limit of $t^{\prime}$ is attained at $\boldsymbol{p}_{p}(\mathrm{RF})=0$,

$$
t^{\prime}<t_{\min }^{\prime} \equiv M_{d}^{2}-2 M_{d} M_{N}=-M_{d} \epsilon_{d}=-0.0041 \mathrm{GeV}^{2} .
$$

Inside the physical region the rest-frame momentum is obtained from $t^{\prime}$ as

$$
\left|\boldsymbol{p}_{p}(\mathrm{RF})\right|^{2}=-\frac{t^{\prime}}{2}\left(1-\frac{t^{\prime}}{2 M_{d}^{2}}\right)+\frac{M_{d}^{2}}{4}-M_{N}^{2} .
$$

A simpler relation is obtained if we neglect the $t^{\prime} /\left(2 M_{d}^{2}\right)$ term in the parentheses; this approximation is well justified for typical 
values $\left|t^{\prime}\right| \sim 0.1 \mathrm{GeV}^{2}$ and becomes exact in the limit $t^{\prime} \rightarrow 0$. Namely,

$$
\begin{aligned}
\left|\boldsymbol{p}_{p}(\mathrm{RF})\right|^{2} & \approx-\frac{t^{\prime}}{2}+\frac{M_{d}^{2}}{4}-M_{N}^{2}=-\frac{t^{\prime}}{2}+\frac{t_{0}^{\prime}}{2}, \\
t_{0}^{\prime} & \equiv \frac{M_{d}^{2}}{2}-2 M_{N}^{2}=-M_{d} \epsilon_{d}-\frac{\epsilon_{d}^{2}}{2} \\
& =-2 M_{N} \epsilon_{d}+\frac{\epsilon_{d}^{2}}{2}=t_{\text {min }}^{\prime}+O\left(\epsilon_{d}^{2}\right) .
\end{aligned}
$$

The difference between $t_{0}^{\prime}$ and the exact $t_{\min }^{\prime}$, Eq. (2.49), is of the order $10^{-6} \mathrm{GeV}^{2}$ and is negligible for all practical purposes. In this approximation the invariant $t^{\prime}$ is the negative of twice the squared rest frame recoil momentum, minus a fixed small amount proportional to the deuteron binding energy,

$$
t^{\prime} \approx-2\left|\boldsymbol{p}_{p}(\mathrm{RF})\right|^{2}+t_{0}^{\prime} .
$$

The relation of the invariant $t^{\prime}$ to the collinear variables $\alpha_{p}$ and $\left|\boldsymbol{p}_{p T}\right|$ can easily be established using the fact that the deuteron rest frame is a special collinear frame $\left(p_{d}^{+}=M_{d}\right)$. The rest-frame energy and $z$ momentum can be calculated in terms of the plus and minus LF components as

$$
\left.\begin{array}{c}
E_{p}(\mathrm{RF}) \\
p_{p}^{z}(\mathrm{RF})
\end{array}\right\}=\frac{p_{p}^{+} \pm p_{p}^{-}}{2}=\frac{\alpha_{p} M_{d}}{4} \pm \frac{\left|\boldsymbol{p}_{p T}\right|^{2}+M_{N}^{2}}{\alpha_{p} M_{d}},
$$

and $t^{\prime}$ can be obtained from the above rest-frame formulas. Specifically, with Eq. (2.53) we obtain

$$
t^{\prime}=-2\left[\left|p_{p}^{z}(\mathrm{RF})\right|^{2}+\left|\boldsymbol{p}_{p T}\right|^{2}\right]+t_{0}^{\prime} .
$$

In the theoretical analysis below we use a representation in which $\alpha_{p}$ and $t^{\prime}$ are independent variables. The physical region in these variables can easily be established from Eq. (2.55). For a given $\alpha_{p}$ the kinematic limit in $t^{\prime}$ is found by minimizing Eq. (2.55) with respect to $\left|\boldsymbol{p}_{p T}\right|$ and is given by (see Fig. 4)

$$
t^{\prime}<-\frac{2 M_{d}^{2}}{\alpha_{p}^{2}}\left(\frac{\alpha_{p}^{2}}{4}-\frac{M_{N}^{2}}{M_{d}^{2}}\right)+t_{0}^{\prime} .
$$

One sees that the minimum value of $-t^{\prime}$ increases quadratically as $\alpha_{p}$ moves away from $2 M_{N} / M_{d} \approx 1$. Conversely, for a given $t^{\prime}<t_{0}^{\prime}$ the allowed values of $\alpha_{p}$ are

$$
\begin{aligned}
\alpha_{1} & <\alpha_{p}<\alpha_{2}, \\
\alpha_{1,2} & =\frac{2}{M_{d}}\left[E_{p}(\mathrm{RF}) \mp\left|\boldsymbol{p}_{p}(\mathrm{RF})\right|\right] \\
& =\frac{2}{M_{d}}\left[\sqrt{\frac{t_{0}^{\prime}-t^{\prime}}{2}+M_{N}^{2}} \mp \sqrt{\frac{t_{0}^{\prime}-t^{\prime}}{2}}\right] .
\end{aligned}
$$

The invariant phase space element in this representation is given by [cf. Eq. (2.48)]

$$
\begin{aligned}
\frac{d^{3} p_{p}}{E_{p}} & =\frac{M_{d}}{8 E_{p}(\mathrm{RF})} d\left(-t^{\prime}\right) d \alpha_{p} d \phi_{p}, \\
E_{p}(\mathrm{RF}) & =\sqrt{\left|\boldsymbol{p}_{p}(\mathrm{RF})\right|^{2}+M_{N}^{2}}=\sqrt{\frac{t_{0}^{\prime}-t^{\prime}}{2}+M_{N}^{2}} .
\end{aligned}
$$

Another physically interesting variable is the angle between the recoil momentum $\boldsymbol{p}_{p}$ and the momentum transfer $\boldsymbol{q}$ in the

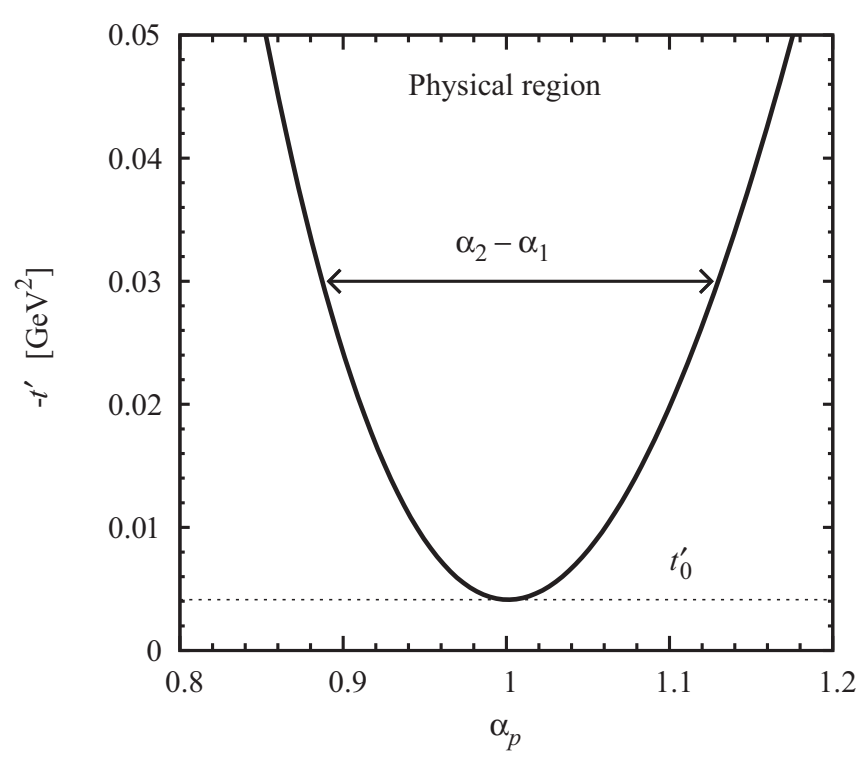

FIG. 4. Physical region of recoil proton momentum phase space in the variables $\alpha_{p}$ and $t^{\prime}$.

deuteron rest frame, $\theta_{p q}$. In our convention $\boldsymbol{q}$ points in the negative $z$ direction (see Fig. 3), so that

$$
\cos \theta_{p q}=-\frac{p_{p}^{z}(\mathrm{RF})}{\left|\boldsymbol{p}_{p}(\mathrm{RF})\right|} .
$$

For a given modulus $\left|\boldsymbol{p}_{p}(\mathrm{RF})\right|$ the angle is related to the LF fraction $\alpha_{p}$ as

$$
\alpha_{p}=\frac{2}{M_{d}}\left(\sqrt{\left|\boldsymbol{p}_{p}(\mathrm{RF})\right|^{2}+M_{N}^{2}}-\left|\boldsymbol{p}_{p}(\mathrm{RF})\right| \cos \theta_{p q}\right) .
$$

The physical region for the angle $\theta_{p q}$ is determined by the condition that $0<\alpha_{p}<2$, which implies

$$
\frac{\sqrt{\left|\boldsymbol{p}_{p}(\mathrm{RF})\right|^{2}+M_{N}^{2}}-M_{d}}{\left|\boldsymbol{p}_{p}(\mathrm{RF})\right|}<\cos \theta_{p q}<1 .
$$

The lower limit becomes larger than -1 only at $\left|\boldsymbol{p}_{p}(\mathrm{RF})\right|>$ $\left(M_{d}^{2}-M_{N}^{2}\right) /\left(2 M_{d}\right) \approx 3 M_{N} / 4$, which is much larger than the recoil momenta considered here, so that effectively all angles are allowed in our kinematics. The regions $\cos \theta_{p q}>0$ and $<0$ are traditionally referred to as the "forward" and "backward" regions.

\section{LIGHT-FRONT QUANTUM MECHANICS}

\section{A. Single-nucleon states}

In our theoretical calculations of the tagged DIS cross section we use methods of LF quantum mechanics. They permit a composite description of nuclear structure in highenergy processes in terms of nucleon degrees of freedom, which can be matched with deep-inelastic nucleon structure and preserves the partonic sum rules [1,11]. In this section we summarize the description of nucleon single-particle states and the deuteron bound state in LF quantum mechanics and the correspondence with the nonrelativistic theory of the deuteron. The LF quantization axis is chosen as the $z$ axis in the collinear 
frame of Sec. II C. The specific dynamical considerations in the application to tagged DIS will be described in Sec. IV B.

In LF quantum mechanics plane-wave nucleon states are characterized by their LF plus and transverse momenta, $p_{N}^{+}=p_{N}^{0}+p_{N}^{z}$ and $\boldsymbol{p}_{N T}=\left(p_{N}^{x}, p_{N}^{y}\right)$ [cf. Eq. (2.32)], while $p_{N}^{-}=p_{N}^{0}-p_{N}^{z}$ plays the role of energy and is fixed by the mass-shell condition $p_{N}^{2}=p_{N}^{+} p_{N}^{-}-\left|\boldsymbol{p}_{N T}\right|^{2}=M_{N}^{2}$,

$$
\left|N, p_{N}\right\rangle \equiv\left|N, p_{N}^{+}, \boldsymbol{p}_{N T}\right\rangle, \quad p_{N}^{-}=\frac{\left|\boldsymbol{p}_{N T}\right|^{2}+M_{N}^{2}}{p_{N}^{+}} .
$$

To simplify the notation we label the states by the fourmomentum $p_{N}$ and display the individual plus and transverse components only if necessary. The relativistic normalization condition for the states is [cf. Eq. (2.9)]

$$
\left\langle N, p_{N}^{\prime} \mid N, p_{N}\right\rangle=(2 \pi)^{3} 2 p_{N}^{+} \delta\left(p_{N}^{+\prime}-p_{N}^{+}\right) \delta\left(\boldsymbol{p}_{N T}^{\prime}-\boldsymbol{p}_{N T}\right) .
$$

The invariant phase space integral over the nucleon LF momentum is

$$
\begin{aligned}
\int d \Gamma_{N}(\ldots) & =\int \frac{d^{4} p_{N}}{(2 \pi)^{4}} 2 \pi \delta\left(p_{N}^{2}-M_{N}^{2}\right) \theta\left(p_{N}^{0}\right)(\ldots) \\
& =\int \frac{d p_{N}^{+} d^{2} p_{N T}}{(2 \pi)^{3} 2 p_{N}^{+}} \theta\left(p_{N}^{+}\right)(\ldots) \\
& {\left[p_{N}^{-}=\left(\left|\boldsymbol{p}_{N T}\right|^{2}+M_{N}^{2}\right) / p_{N}^{+}\right] } \\
& \equiv \int\left[d p_{N}\right](\ldots) .
\end{aligned}
$$

The condition $p_{N}^{+}>0$ is satisfied for all physical nucleon momenta. The completeness of single-nucleon states can then be stated in the form

$$
\int\left[d p_{N}\right]\left|N, p_{N}\right\rangle\left\langle N, p_{N}\right|=1_{N},
$$

which is the unit operator in the single-nucleon space. For reference we note that for a general four-momentum $p$ (not on mass shell), the four-dimensional integral and the four-dimensional delta function in LF components take the form

$$
\begin{aligned}
\int d^{4} p(\ldots) & =\frac{1}{2} \int_{-\infty}^{\infty} d p^{+} \int_{-\infty}^{\infty} d p^{-} \int d^{2} p_{T}(\ldots), \\
\delta^{(4)}(p) & =2 \delta\left(p^{+}\right) \delta\left(p^{-}\right) \delta^{(2)}\left(\boldsymbol{p}_{T}\right) .
\end{aligned}
$$

The description of the nucleon's spin degrees of freedom in LF quantum mechanics is summarized in Sec. IIID. Other hadronic states are described in a similar fashion.

\section{B. Deuteron wave function}

In the LF description of high-energy processes nuclei are described as bound states of nucleons and, possibly, non-nucleonic degrees of freedom ( $\Delta$ isobars, pions) $[1,11]$. Theoretical arguments show that for the deuteron the nucleonic ( $p n)$ component dominates over a wide range of excitation energies [11] (see also Sec. I), and we limit ourselves to this component in the present study. The deuteron is described as a bound state with relativistic normalization of the CM motion

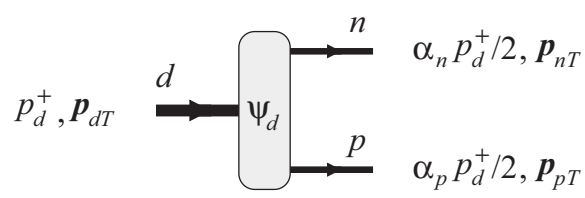

FIG. 5. Deuteron $p n$ LF wave function.

[cf. Eq. (3.2)]

$$
\begin{aligned}
& \left|d, p_{d}\right\rangle, \quad p_{d}^{-}=\frac{\left|\boldsymbol{p}_{d T}\right|^{2}+M_{d}^{2}}{p_{d}^{+}} \\
& \left\langle d, p_{d}^{\prime} \mid d, p_{d}\right\rangle=(2 \pi)^{3} 2 p_{d}^{+} \delta\left(p_{d}^{+\prime}-p_{d}^{+}\right) \delta^{(2)}\left(\boldsymbol{p}_{d T}^{\prime}-\boldsymbol{p}_{d T}\right) .
\end{aligned}
$$

The expansion of the deuteron state in plane-wave nucleon states is described by the LF wave function (see Fig. 5)

$$
\begin{aligned}
\left\langle p, p_{p} ; n, p_{n} \mid d, p_{d}\right\rangle= & (2 \pi)^{3} 2 p_{d}^{+} \delta\left(p_{p}^{+}+p_{n}^{+}-p_{d}^{+}\right) \\
& \times \delta^{(2)}\left(\boldsymbol{p}_{p T}+\boldsymbol{p}_{n T}-\boldsymbol{p}_{d T}\right) \\
& \times(2 \pi)^{3 / 2} \Psi_{d}\left(\alpha_{p}, \boldsymbol{p}_{p T} \mid \boldsymbol{p}_{d T}\right) \\
\alpha_{p} \equiv & \frac{2 p_{p}^{+}}{p_{d}^{+}}, \quad \alpha_{n} \equiv \frac{2 p_{n}^{+}}{p_{d}^{+}}
\end{aligned}
$$

The factor $(2 \pi)^{3 / 2}$ is conventional. The function $\Psi_{d}$ depends on the LF momentum fraction and the transverse momentum of the proton, $\alpha_{p}$ and $\boldsymbol{p}_{p T}$, and the deuteron transverse momentum $\boldsymbol{p}_{d T}$; it is independent of the total plus momentum $p_{d}^{+}$because of longitudinal boost invariance $[11,35]$. The delta functions in Eq. (3.9) require that

$$
\alpha_{p}+\alpha_{n}=2, \quad \boldsymbol{p}_{p T}+\boldsymbol{p}_{n T}=\boldsymbol{p}_{d T},
$$

which in particular implies that

$$
0<\alpha_{p}<2, \quad 0<\alpha_{n}<2 .
$$

The matrix element Eq. (3.9) is symmetric with respect to interchange of the proton and neutron, and the wave function satisfies

$$
\begin{aligned}
\Psi_{d}\left(\alpha_{p}, \boldsymbol{p}_{p T} \mid \boldsymbol{p}_{d T}\right) & =\Psi_{d}\left(2-\alpha_{p}, \boldsymbol{p}_{d T}-\boldsymbol{p}_{p T} \mid \boldsymbol{p}_{d T}\right) \\
& =\Psi_{d}\left(\alpha_{n}, \boldsymbol{p}_{n T} \mid \boldsymbol{p}_{d T}\right) .
\end{aligned}
$$

The normalization of the deuteron wave function follows from the normalization condition for the deuteron state Eq. (3.8) and the completeness relation for the single-nucleon states Eq. (3.5). Inserting complete sets of single-nucleon intermediate states into Eq. (3.8) and integrating out the delta functions one obtains

$$
\int \frac{d \alpha_{p} d^{2} p_{p T}}{\alpha_{p}\left(2-\alpha_{p}\right)}\left|\Psi_{d}\left(\alpha_{p}, \boldsymbol{p}_{p T} \mid \boldsymbol{p}_{d T}\right)\right|^{2}=1 .
$$

In the calculations of deuteron structure in the collinear frame of Sec. IIC we need the wave function at zero deuteron transverse momentum, which we denote by

$$
\Psi_{d}\left(\alpha_{p}, \boldsymbol{p}_{p T}\right) \equiv \Psi_{d}\left(\alpha_{p}, \boldsymbol{p}_{p T} \mid \boldsymbol{p}_{d T}=0\right) .
$$


The symmetry relation Eq. (3.13) for this function takes the simple form

$$
\Psi_{d}\left(\alpha_{p}, \boldsymbol{p}_{p T}\right)=\Psi_{d}\left(2-\alpha_{p},-\boldsymbol{p}_{p T}\right)=\Psi_{d}\left(\alpha_{n}, \boldsymbol{p}_{n T}\right) .
$$

The inclusion of the nucleon's and deuteron's spin degrees of freedom is described in Sec. IIID.

For modeling the actual form of the deuteron LF wave function it is natural to consider the connection of the LF formulation with the nonrelativistic description of deuteron structure. In general this connection is rather complicated, because of the different symmetry groups of the dynamics in the two formulations. However, a simple connection can be established in the approximation where the deuteron's LF structure is restricted to the $p n$ component, which we adopt here [1,11]. One starts with the LF version of the Lippmann-Schwinger equation for the two-body wave function (or Weinberg equation [50]) and imposes the condition that the scattering-state solutions give rotationally invariant on-shell $N N$ scattering amplitudes (angular conditions) [1,11]. The resulting equation for bound states has a simple connection with the Schrödinger equation for the nonrelativistic deuteron wave function, which one can use to construct an approximation of the LF wave function in terms of the nonrelativistic wave function (see Sec. IIIC). Methods for direct solution of the LF two-body bound-state equation have been described in Refs. [51,52]. For attempts to model deuteron LF structure beyond the $p n$ component, and for approximation methods for heavier nuclei, we refer to Refs. [53,54] and references therein.

\section{Rotationally invariant representation}

The LF wave function of a two-body bound state such as the deuteron can be expressed in a form that exhibits three-dimensional rotational invariance $[1,11,55]$. This representation can be motivated by group-theoretical or dynamical considerations (see above) and is useful for several purposes: (a) it explains how rotational invariance is dynamically realized in LF quantum mechanics, where it is not manifest (angular conditions); (b) it enables an approximation of the LF wave function in terms of the three-dimensional nonrelativistic wave function; (c) it brings out the analytic properties of the LF wave function in the nucleon momentum.

The rotationally invariant momentum variable for the twonucleon system can be introduced through an intuitive procedure, by identifying the $p n$ configurations in the deuteron LF wave function with a free $p n$ system in its CM frame [11]. One starts from a $p n$ configuration in its CM frame, with the proton having LF momentum $\alpha_{p}$ and $\boldsymbol{p}_{p T}$, calculates the invariant mass of the $p n$ configuration, and equates the invariant mass with the squared energy of a free $p n$ system with relative three-momentum

$$
\boldsymbol{k} \equiv\left(\boldsymbol{k}_{T}, k^{z}\right), \quad \boldsymbol{k}_{T}=\boldsymbol{p}_{T} .
$$

This leads to the equation

$$
s_{p n} \equiv \frac{\boldsymbol{p}_{p T}^{2}+M_{N}^{2}}{\frac{\alpha_{p}}{2}\left(1-\frac{\alpha_{p}}{2}\right)} \stackrel{!}{=} 4 E_{N}(\boldsymbol{k})^{2}=4\left(|\boldsymbol{k}|^{2}+M_{N}^{2}\right) \text {. }
$$

One then determines the component $k^{z}$ as function of $\alpha_{p}$ and $\left|\boldsymbol{p}_{p T}\right|$ by solving Eq. (3.18),

$$
k^{z}=\left(\alpha_{p}-1\right)\left[\frac{p_{p T}^{2}+M_{N}^{2}}{\alpha_{p}\left(2-\alpha_{p}\right)}\right]^{1 / 2} .
$$

Equations (3.17) and (3.19) define the equivalent threemomentum $\boldsymbol{k}$ in terms of the LF variables of the two-body system. The inverse relation is

$$
\alpha_{p}=1+\frac{k^{z}}{E_{N}}=\frac{2\left(E_{N}+k^{z}\right)}{2 E_{N}}, \quad E_{N} \equiv \sqrt{|\boldsymbol{k}|^{2}+M_{N}^{2}} .
$$

Note that in this parametrization the nucleon plus momentum fraction is obtained by dividing $E_{N}+k^{z}$ by the internal energy of the pn system, $2 E_{N}$, not by the external mass of the bound state, as in the kinematic variable Eq. (2.43). The invariant phase space elements in the two sets of variables are related as

$$
\frac{d \alpha_{p} d^{2} p_{p T}}{\alpha_{p}\left(2-\alpha_{p}\right)}=\frac{d^{3} k}{\sqrt{|\boldsymbol{k}|^{2}+M_{N}^{2}}}=\frac{d^{3} k}{E_{N}(\boldsymbol{k})} .
$$

The rotationally invariant form of the deuteron LF wave function is then obtained by demanding that

$$
\Psi_{d}\left(\alpha_{p}, \boldsymbol{p}_{p T}\right)=\text { function }(|\boldsymbol{k}|) .
$$

It was shown in Ref. [11] that this condition is sufficient to guarantee rotational invariance in two-body bound state calculations.

The rotationally symmetric form Eq. (3.22) implies a connection of the rest-frame deuteron LF wave function with the nonrelativistic wave function [11]:

$$
\Psi_{d}\left(\alpha_{p}, \boldsymbol{p}_{p T}\right)=\sqrt{M_{N}} \widetilde{\Psi}_{d}(\boldsymbol{k})+O\left(\left|\boldsymbol{p}_{p}\right|^{2} / M_{N}^{2}\right),
$$

where $\widetilde{\Psi}_{d}$ denotes the nonrelativistic wave function and the arguments are related by Eqs. (3.17) and (3.19). Rotational symmetry-breaking corrections of linear order $O\left(\left|\boldsymbol{p}_{p}\right| / M_{N}\right)$ are accounted for by the argument $\boldsymbol{k}$, so that the relation is accurate to quadratic order in the proton momentum. The factor $\sqrt{M_{N}}$ accounts for the different normalization of the LF and nonrelativistic wave functions.

The rotationally invariant representation Eq. (3.22) is also sufficient for ensuring the correct analytic properties of the LF wave function at small relative momenta (nucleon pole). We can demonstrate this using the approximation Eq. (3.23), which becomes exact at small recoil momenta. On general grounds the nonrelativistic deuteron wave function has a pole at small unphysical momenta,

$$
\begin{aligned}
\widetilde{\Psi}_{d}(\boldsymbol{k}) & \sim \frac{\Gamma}{|\boldsymbol{k}|^{2}+a^{2}} \quad\left(|\boldsymbol{k}|^{2} \rightarrow-a^{2}\right), \\
a^{2} & =M_{N} \epsilon_{d}-\frac{\epsilon_{d}^{2}}{4} .
\end{aligned}
$$

The pole results from the free propagation of the nucleons outside the range of the $p n$ interaction and controls the largedistance behavior of the coordinate-space wave function. [In the Bethe-Peierls approximation the entire deuteron wave function is given by Eq. (3.24).] By expressing $\left|\boldsymbol{k}^{2}\right|$ in Eq. (3.24) 
in terms of the LF momentum variables using Eq. (3.18), one easily sees that the pole corresponds to a pole in the invariant mass $s_{p n}$ of the LF wave function,

$$
\Psi_{d}\left(\alpha_{p}, \boldsymbol{p}_{p T}\right) \sim \frac{4 \sqrt{M_{N}} \Gamma}{s_{p n}-M_{d}^{2}} .
$$

The singularity Eq. (3.24) viz. Eq. (3.26) gives rise to the nucleon pole in the deuteron spectral function and plays an essential role in the theory of tagged DIS (see below).

The above results can be used to derive an approximate expression for the LF wave function directly in terms of the rest-frame proton momentum $\boldsymbol{p}_{p}$. Expanding the variable $\boldsymbol{k}$ in powers of

$$
\left|\boldsymbol{p}_{p}\right| / M_{N}, \quad p_{p}^{z} / M_{N},
$$

and counting the binding energy as $\epsilon_{d} M_{N}=O\left(p_{p}^{2}\right)$, one obtains

$$
|\boldsymbol{k}|^{2}+a^{2}=\left(\left|\boldsymbol{p}_{p}\right|^{2}+a^{2}\right)\left(1+\frac{p^{z}}{M_{N}}\right)+O\left(p_{p}^{4}\right) \quad\left(a^{2} \equiv \epsilon_{d} M_{N}\right) .
$$

In the region where the nonrelativistic deuteron wave function is dominated by the nucleon pole, Eq. (3.24), one can therefore approximate the LF wave function Eq. (3.23) as

$$
\Psi_{d}\left(\alpha_{p}, \boldsymbol{p}_{p T}\right)=\sqrt{M_{N}}\left(1-\frac{p^{z}}{M_{N}}\right) \widetilde{\Psi}_{d}\left(\boldsymbol{p}_{p}\right) .
$$

The breaking of rotational invariance is now explicit in the $p^{z}$-dependent prefactor. Numerical studies show that Eq. (3.29) approximates the LF wave function well up to momenta $p_{p} \sim$ $200 \mathrm{MeV}$. We use this approximation in the following studies, including also the spin degrees of freedom.

\section{Spin degrees of freedom}

In LF quantum mechanics the spin-1/2 nucleon is described by LF helicity states

$$
\begin{aligned}
\left|p_{N}, \sigma_{N}\right\rangle \equiv\left|p_{N}^{+}, \boldsymbol{p}_{N T}, \sigma_{N}\right\rangle, \quad \sigma_{N}=\left(-\frac{1}{2},+\frac{1}{2}\right), \\
\left\langle N, p_{N}^{\prime}, \sigma_{N}^{\prime} \mid N, p_{N}, \sigma_{N}\right\rangle=(2 \pi)^{3} 2 p_{N}^{+} \delta\left(p_{N}^{+\prime}-p_{N}^{+}\right) \\
\times \delta\left(\boldsymbol{p}_{N T}^{\prime}-\boldsymbol{p}_{N T}\right) \delta\left(\sigma_{N}^{\prime}, \sigma_{N}\right) .
\end{aligned}
$$

They are obtained by preparing states quantized in the $z$ direction in the nucleon rest frame, then performing a longitudinal boost to the desired plus momentum $p_{N}^{+}$, then a transverse boost to the desired transverse momentum $\boldsymbol{p}_{N T}$ [33-36]. The states thus defined are invariant under longitudinal boosts and transform kinematically under transverse boosts, and coincide with the usual $z$-quantized spin states in the nonrelativistic limit. Likewise, the spin-1 deuteron is described by LF helicity states

$$
\left|p_{d}^{+}, \boldsymbol{p}_{d T}, \lambda_{d}\right\rangle, \quad \lambda_{d}=(-1,0,1)
$$

which are obtained in a similar fashion. The expansion of the deuteron state in nucleon states, Eq. (3.9), now takes the form

$$
\begin{aligned}
& \left\langle p, p_{p}, \sigma_{p} ; n, p_{n}, \sigma_{n} \mid d, p_{d}, \lambda_{d}\right\rangle \\
& \quad=[\ldots](2 \pi)^{3 / 2} \Psi_{d}\left(\alpha_{p}, \boldsymbol{p}_{p T} ; \sigma_{p}, \sigma_{n} \mid \boldsymbol{p}_{d T}, \lambda_{d}\right),
\end{aligned}
$$

where $[\ldots]$ stands for the momentum delta functions of Eq. (3.9). The LF wave function depends on the LF helicities of the proton and neutron as well as the deuteron, and is normalized such that

$$
\begin{aligned}
& \sum_{\sigma_{p}, \sigma_{n}} \int \frac{d \alpha_{p} d^{2} p_{p T}}{\alpha_{p}\left(2-\alpha_{p}\right)} \Psi_{d}^{*}\left(\alpha_{p}, \boldsymbol{p}_{p T} ; \sigma_{p}, \sigma_{n} \mid \boldsymbol{p}_{d T}, \lambda_{d}^{\prime}\right) \\
& \quad \times \Psi_{d}\left(\alpha_{p}, \boldsymbol{p}_{p T} ; \sigma_{p}, \sigma_{n} \mid \boldsymbol{p}_{d T}, \lambda_{d}\right)=\delta\left(\lambda_{d}^{\prime}, \lambda_{d}\right) .
\end{aligned}
$$

The spin structure of the deuteron is well understood in the nonrelativistic theory. The deuteron wave function in the rest frame depends on the spin projections of the nucleons and the deuteron,

$$
\widetilde{\Psi}_{d}\left(\boldsymbol{p}_{p} ; \sigma_{p}, \sigma_{n} \mid \lambda_{d}\right) .
$$

The wave function has $S$ - and $D$-wave components (orbital angular momentum $L=0$ and 2); in both components the nucleon spins are in the spin-triplet state (total nucleon spin $S=1$ ), and the total nucleon spin is coupled with the orbital angular momentum to deuteron spin 1 . An explicit expression for the nonrelativistic wave function is given in Appendix A. The $S$ wave has a total probability $\sim 95 \%$, contains the nucleon pole Eq. (3.24) at small momenta, and dominates at momenta up to $\left|\boldsymbol{p}_{p}\right| \sim 200 \mathrm{MeV}$; the $D$ wave has total probability $\sim 5 \%$ and becomes important at larger momenta.

The nonrelativistic approximation to the LF wave function in the rest frame, Eq. (3.29), now takes the form

$$
\begin{aligned}
\Psi_{d}\left(\alpha_{p}, \boldsymbol{p}_{p T} ; \sigma_{p}, \sigma_{n} \mid \lambda_{d}\right)= & \sum_{\sigma_{p}^{\prime}, \sigma_{n}^{\prime}} U\left(\sigma_{p}, \sigma_{p}^{\prime}\right) U\left(\sigma_{n}, \sigma_{n}^{\prime}\right) \sqrt{M_{N}} \\
& \times\left(1-\frac{p^{z}}{M_{N}}\right) \widetilde{\Psi}_{d}\left(\boldsymbol{p}_{p} ; \sigma_{p}^{\prime}, \sigma_{n}^{\prime} \mid \lambda_{d}\right) .
\end{aligned}
$$

Here $U$ are the spin rotation matrices, which describe the transformation between the nonrelativistic spin and the LF helicity to first order in the momentum; their explicit form is given in Ref. [35]. In the following applications the spin rotations drop out because of summation over the spins and do not need to be considered explicitly. We can therefore effectively identify the LF helicity with the $z$ projection of the nonrelativistic spin, and write Eq. (3.36) in the form

$$
\begin{aligned}
& \Psi_{d}\left(\alpha_{p}, \boldsymbol{p}_{p T} ; \sigma_{p}, \sigma_{n} \mid \lambda_{d}\right) \\
& \quad=\sqrt{M_{N}}\left(1-\frac{p^{z}}{M_{N}}\right) \widetilde{\Psi}_{d}\left(\boldsymbol{p}_{p} ; \sigma_{p}, \sigma_{n} \mid \lambda_{d}\right) .
\end{aligned}
$$

For practical calculations we use Eq. (3.37) with the nonrelativistic deuteron wave function obtained from the AV18 NN potential [56] (see Appendix A).

\section{IMPULSE APPROXIMATION}

\section{A. LF current components}

We now compute the cross section for tagged DIS on the deuteron using LF quantum mechanics. The basic considerations in treating nuclear structure in high-energy scattering are described in Refs. $[1,11]$ and summarized in Sec. I. In LF quantization the effects of the off-shellness of the constituents 
in a bound state remain finite as the scattering energy tends to infinity, which makes possible a composite description of the nucleus in terms of nucleon degrees of freedom (see below). We use the collinear frame of Sec. IIC $\left(\boldsymbol{p}_{d T}=0, \boldsymbol{q}_{T}=0\right)$, in which the initial nucleus and the DIS final state evolve along the same $z$-direction, as this permits a natural description of FSIs with rotational invariance in the transverse plane. Noncollinear frames with $\boldsymbol{q}_{T} \neq 0$ can be used for LF calculations of the inclusive DIS cross section but are not suitable for FSIs [1]. In the collinear frame the momentum transfer to the nucleus has LF component $q^{+}<0$, Eq. (2.33), so that the current cannot produce physical hadron states out of the vacuum, but can only couple to nucleons in the nuclear LF wave function.

In order to extract the tagged deuteron structure functions of Eq. (2.22) in the collinear frame we must calculate both + and $T$ components of the nuclear tensor. It is well known that in LF quantization the different components of the current operator have a different status as to how they involve the interactions of the system. This is seen explicitly in the LF quantization of quantum field theories, where only two components of the spin-1/2 Dirac field are independent canonical degrees of freedom, while the other two are dependent and must be eliminated through the equations of motion $[57,58]$. The "good" current $J^{+}$is formed out of canonical degrees of freedom and free of interactions; the "bad" current $\boldsymbol{J}_{T}$ is formed out of canonical and dependent degrees of freedom and involves explicit interactions; the "worst" current $J^{-}$is formed entirely out of dependent degrees of freedom. ${ }^{2}$ Following Refs. [1,11] we calculate the $J^{+}$and $\boldsymbol{J}_{T}$ matrix elements in our approach (IA and FSI); the $J^{-}$component can be eliminated through the transversality condition in the collinear frame (current conservation) and does not need to be considered explicitly,

$$
q^{\mu}\left\langle B\left|J_{\mu}\right| A\right\rangle=\frac{q^{+}}{2}\left\langle B\left|J^{-}\right| A\right\rangle+\frac{q^{-}}{2}\left\langle B\left|J^{+}\right| A\right\rangle=0
$$

The use of the component $\boldsymbol{J}_{T}$ for structure function calculations represents an approximation whose accuracy cannot

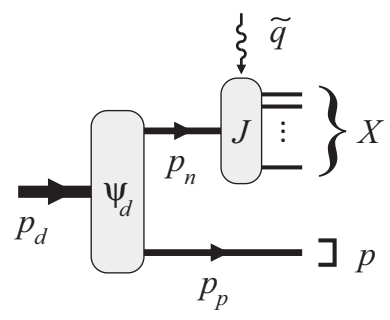

FIG. 6. Current matrix element of tagged DIS on the deuteron in the IA.

be established from first principles in our phenomenological approach. In inclusive DIS, comparison between a goodcurrent calculation in a noncollinear frame and the goodand-bad-current calculation in the collinear frame shows that the two schemes give the same results in the DIS limit $\left(\operatorname{mass}^{2}\right) / W^{2} \rightarrow 0$ [1]. This indicates that the collinear method should be reliable for the leading-twist tagged structure functions $F_{2 d}$ and $F_{L d}$ calculated in this work. A further test of the method will be performed in Sec. IVC. The applicability to higher-twist structure functions, which represent powersuppressed structures in the tagged cross section, remains to be investigated.

\section{B. IA current}

The starting point of the nuclear structure calculation is the IA. Its physical assumptions are (a) the current operator is the sum of one-body nucleon currents, and (b) the final state produced by the one-body nucleon current evolves independently of a nuclear remnant (see Fig. 6) [59]. In the IA we consider the nuclear current matrix element in Eq. (2.19) in the collinear frame $\left(\boldsymbol{p}_{d T}=0\right)$ and insert plane-wave proton and neutron states between the deuteron state and the current operator. Taking the proton as the spectator, and the neutron as coupling to the current, we obtain (see Fig. 6)

$$
\begin{aligned}
\left\langle p X\left|\hat{J}^{\mu}(0)\right| d\right\rangle[\mathrm{IA}] & =\int\left[d p_{p 1}\right] \int\left[d p_{n}\right]\left\langle X\left|\hat{J}^{\mu}(0)\right| n, p_{n}\right\rangle\left\langle p, p_{p} \mid p, p_{p 1}\right\rangle\left\langle p, p_{p 1} ; n, p_{n} \mid d, p_{d}\right\rangle \\
& =(2 \pi)^{3 / 2} \frac{p_{d}^{+}}{p_{n}^{+}}\left\langle X\left|\hat{J}^{\mu}(0)\right| n, p_{n}\right\rangle \Psi_{d}\left(\alpha_{p}, \boldsymbol{p}_{p T}\right) \quad\left(p_{n}^{+}=p_{d}^{+}-p_{p}^{+}, \boldsymbol{p}_{n T}=-\boldsymbol{p}_{p T}\right) .
\end{aligned}
$$

The deuteron tensor Eq. (2.19) then becomes

$$
\begin{aligned}
W_{d}^{\mu \nu}\left(p_{d}, q ; p_{p}\right)= & \left(\frac{p_{d}^{+}}{p_{n}^{+}}\right)^{2}(2 \pi)^{3}\left|\Psi_{d}\left(\alpha_{p}, \boldsymbol{p}_{p T}\right)\right|^{2} \\
& \times(4 \pi)^{-1} \sum_{X}(2 \pi)^{4} \delta^{(4)}\left(q+p_{d}-p_{p}-p_{X}\right)\left\langle n, p_{n}\left|\hat{J}^{\mu}(0)\right| X\right\rangle\left\langle X\left|\hat{J}^{\nu}(0)\right| n, p_{n}\right\rangle \\
& \left(p_{n}^{+}=p_{d}^{+}-p_{p}^{+}, \boldsymbol{p}_{n T}=-\boldsymbol{p}_{p T}\right) .
\end{aligned}
$$

\footnotetext{
${ }^{2}$ In the equivalent formulation based on equal-time quantization in the infinite-momentum frame $|\boldsymbol{p}| \rightarrow \infty$, the "good" components are those that tend to a finite limit as $|\boldsymbol{p}| \rightarrow \infty$ (in the noncovariant normalization of states), while the other components vanish.
} 
The expression on the second line has a form suggestive of the scattering tensor for inclusive scattering on the neutron. However, we must take into account that in LF quantum mechanics four-momenta are not conserved, and that

$$
p_{d}-p_{p} \neq p_{n} \quad \text { (four-vectors) }
$$

in the argument of the four-dimensional delta function. This is because the LF energy of the neutron is determined by the mass shell condition

$$
p_{n}^{-}=\frac{\left|\boldsymbol{p}_{n T}\right|^{2}+M_{N}^{2}}{p_{n}^{+}} \neq p_{d}^{-}-p_{p}^{-} .
$$

The expression in Eq. (4.4) can therefore not be regarded as the neutron scattering tensor with the original four-momentum transfer $q$, which is fixed kinematically by the electron fourmomenta. To write it as a proper scattering tensor we define an effective four-momentum transfer as

$$
\begin{aligned}
& \widetilde{q} \equiv q+p_{d}-p_{n}-p_{p}, \quad \text { or } \quad p_{n}+\widetilde{q}=q+p_{d}-p_{p} \\
& {\left[p_{n}^{+}=p_{d}^{+}-p_{p}^{+}, \boldsymbol{p}_{n T}=-\boldsymbol{p}_{p T}, p_{n}^{-}=\left(\left|\boldsymbol{p}_{n T}\right|^{2}+M_{N}^{2}\right) / p_{n}^{+}\right] .}
\end{aligned}
$$

The vector $\widetilde{q}$ has the same plus and transverse components as the original $q$ and differs only in its minus component, which is not conserved in LF quantum mechanics. The difference accounts for the fact that the $p n$ configurations in the deuteron are off the LF energy shell and participate in the scattering process with shifted kinematics. With the effective momentum transfer Eq. (4.7) the deuteron tensor Eq. (4.4) can then be expressed in terms of the effective neutron tensor [we use $\left.p_{d}^{+} / p_{n}^{+}=2 /\left(2-\alpha_{p}\right)\right]$

$$
\begin{aligned}
W_{d}^{\mu \nu}\left(p_{d}, q, p_{p}\right)= & \left(\frac{p_{d}^{+}}{p_{n}^{+}}\right)^{2}(2 \pi)^{3}\left|\Psi_{d}\left(\alpha_{p}, \boldsymbol{p}_{p T}\right)\right|^{2} W_{n}^{\mu \nu}\left(p_{n}, \widetilde{q}\right) \\
= & 2(2 \pi)^{3} \frac{2\left|\Psi_{d}\left(\alpha_{p}, \boldsymbol{p}_{p T}\right)\right|^{2}}{\left(2-\alpha_{p}\right)^{2}} W_{n}^{\mu v}\left(p_{n}, \widetilde{q}\right), \quad(4.9) \\
W_{n}^{\mu \nu}\left(p_{n}, \widetilde{q}\right) \equiv & (4 \pi)^{-1} \sum_{X}(2 \pi)^{4} \delta^{(4)}\left(\widetilde{q}+p_{n}-p_{X}\right) \\
& \times\left\langle n, p_{n}\left|\hat{J}^{\mu}(0)\right| X\right\rangle\left\langle X\left|\hat{J}^{v}(0)\right| n, p_{n}\right\rangle .
\end{aligned}
$$

Equation (4.9) represents the "master formula" for tagged DIS in the LF IA and expresses the factorization of deuteron and nucleon structure.

The assignment of the active nucleon four-momentum $p_{n}$ as in Eq. (4.6) and of the four-momentum transfer $\widetilde{q}$ as in Eq. (4.7) are dictated by LF quantum mechanics, in which the $\mathrm{LF}+$ and $T$ momenta are conserved and the particles are on mass shell, but the total LF energy of the intermediate states is different from that of the initial and final states. A crucial point is that in this scheme the nonconservation of four-momentum does not give rise to any large invariants in the DIS limit $W^{2} \rightarrow \infty, Q^{2} \rightarrow \infty, Q^{2} / W^{2}$ fixed. The off-shellness of the minus component of the nucleon four-momentum implied by
Eq. (4.6) is

$$
\begin{aligned}
p_{n}^{-}-p_{d}^{-}+p_{p}^{-} & =\frac{\left|\boldsymbol{p}_{p T}\right|^{2}+M_{N}^{2}}{p_{n}^{+}}-\frac{M_{d}^{2}}{p_{d}^{+}}+\frac{\left|\boldsymbol{p}_{p T}\right|^{2}+M_{N}^{2}}{p_{p}^{+}} \\
& =\frac{1}{p_{d}^{+}}\left[\frac{4\left(\left|\boldsymbol{p}_{p T}\right|^{2}+M_{N}^{2}\right)}{\alpha_{p}\left(2-\alpha_{p}\right)}-M_{d}^{2}\right],
\end{aligned}
$$

where we have used the explicit expressions for the LF momentum components in the collinear frame of Sec. II C. The plus component of the momentum transfer is $q^{+}=-\xi_{d} p_{d}^{+}$, cf. Eq. (2.33). The variables $\alpha_{p},\left|\boldsymbol{p}_{p T}\right|$ and $\xi_{d}$ remain finite in the DIS limit, and $p_{d}^{+}$is a finite boost parameter. One therefore has

$$
\begin{aligned}
2 q\left(p_{n}-p_{d}+p_{p}\right) & =q^{+}\left(p_{n}^{-}-p_{d}^{-}+p_{p}^{-}\right) \\
& =O\left\{\left|p_{p T}\right|^{2},(\text { mass })^{2}\right\}
\end{aligned}
$$

i.e., the invariant remains finite and does not grow as $W^{2}$ or $Q^{2}$. (Note that individually $q p_{d} \sim W^{2}$ and $q p_{n} \sim W^{2}$, because $p_{d}$ and $p_{n}$ have nonzero plus components.) It implies that the effects caused by the LF energy off-shellness are power suppressed as $\sim\left|\boldsymbol{p}_{p T}\right|^{2} / W^{2}$ or $\sim(\text { mass })^{2} / W^{2}$ in the DIS limit. This circumstance is unique about LF quantization and is the reason for the use of this approach in high-energy scattering.

\section{Structure functions}

Expressions for the tagged deuteron structure functions are obtained from Eq. (4.9) by substituting the specific form of the neutron tensor and projecting the tensor equation on the structures of Eq. (2.22). The decomposition of the neutron tensor is analogous to that of the deuteron tensor Eq. (2.22), but with the target four-momentum given by $p_{n}$, and the four-momentum transfer given by $\widetilde{q}$,

$$
\begin{aligned}
W_{n}^{\mu \nu}\left(p_{n}, \widetilde{q}\right)= & \left(g^{\mu \nu}-\frac{\widetilde{q}^{\mu} \widetilde{q}^{v}}{\widetilde{q}^{2}}\right) \frac{F_{L n}\left(\widetilde{x}, \widetilde{Q}^{2}\right)}{2} \\
& +\left(\frac{\widetilde{L}_{n}^{\mu} \widetilde{L}_{n}^{v}}{\widetilde{L}_{n}^{2}}+\frac{\widetilde{q}^{\mu} \widetilde{q}^{v}}{\widetilde{q}^{2}}-g^{\mu \nu}\right) \frac{F_{T n}\left(\widetilde{x}, \widetilde{Q}^{2}\right)}{2}, \\
F_{T n}= & \frac{\left(-\widetilde{q}^{2}\right) L_{n}^{2}}{\left(p_{n} \widetilde{q}\right)^{2}} \frac{F_{2 n}}{\widetilde{x}}=\left(1+\frac{4 \widetilde{x}^{2} M_{N}^{2}}{\widetilde{Q}^{2}}\right) \frac{F_{2 n}}{\widetilde{x}}, \quad(4.15) \\
\widetilde{L}_{n}^{\mu} \equiv & p_{n}^{\mu}-\frac{\left(p_{n} \widetilde{q}\right) \widetilde{q}^{\mu}}{\widetilde{q}^{2}}, \quad \widetilde{x} \equiv \frac{-\widetilde{q}^{2}}{2\left(p_{n} \widetilde{q}\right)}, \quad \widetilde{Q}^{2} \equiv-\widetilde{q}^{2} .
\end{aligned}
$$

Equations for the structure functions can then be derived by considering the + and $T$ tensor components in the collinear frame (see Appendix B). They take on a simple form in the DIS limit, where one can neglect terms of the order $\left|\boldsymbol{p}_{p T}\right|^{2} / W^{2}$ and (mass) ${ }^{2} / W^{2}$, so that off-shell effects are suppressed [cf. Eq. (4.13)]. In particular, in this limit

$$
\widetilde{Q}^{2}=Q^{2}, \quad \tilde{x}=\frac{x}{2-\alpha_{p}},
$$


up to power corrections, i.e., the nucleon structure functions are evaluated at the kinematically given $Q^{2}$, and at an effective value of $x$ that accounts for the longitudinal motion of the nucleons in the bound state. Altogether we obtain

$$
\begin{aligned}
& F_{2 d}\left(x, Q^{2} ; \alpha_{p}, p_{p T}\right)=\frac{\left|\Psi_{d}\left(\alpha_{p}, \boldsymbol{p}_{p T}\right)\right|^{2}}{2-\alpha_{p}} F_{2 n}\left(\tilde{x}, Q^{2}\right), \\
& F_{L d}\left(x, Q^{2} ; \alpha_{p}, p_{p T}\right)=\frac{2\left|\Psi_{d}\left(\alpha_{p}, \boldsymbol{p}_{p T}\right)\right|^{2}}{\left(2-\alpha_{p}\right)^{2}} F_{L n}\left(\tilde{x}, Q^{2}\right) .
\end{aligned}
$$

These formulas express the deuteron DIS structure functions with tagged proton in terms of the deuteron LF momentum density and the active neutron inclusive structure functions. The case of tagged neutron and active proton is described by the same formulas with the proton and neutron labels interchanged.

Our calculation in the collinear frame uses both good and bad LF current components to identify the structure functions (cf. Sec. IV A). The results for the bad current component in the LF IA are generally sensitive to the energy off-shellness (four-momentum nonconservation) in the intermediate state. These effects are related to those of explicit interactions in the bad current component operators. In a complete dynamical theory both could be treated consistently starting from the microscopic interaction. To assess their influence within our phenomenological approach we perform a simple test, following Ref. [1]. We evaluate Eq. (4.9) with the neutron tensor $W_{n}^{\mu \nu}$ taken at the off-mass-shell four-momentum $\widetilde{p}_{n} \equiv$ $p_{d}-p_{p}$ with $\widetilde{p}_{n}^{2} \neq M_{N}^{2}$, as would by obtained from the external four-momenta using four-momentum conservation, and at the original momentum transfer $q$ ("virtual nucleon"). We compare the results with those of the LF prescription, where $W_{n}^{\mu \nu}$ is evaluated at $p_{n}$ and $\widetilde{q}$, Eqs. (4.6) and (4.7). The differences in the leading-twist tagged structure functions $F_{2 d}$ and $F_{L d}$ turn out to be of the order $\left|\boldsymbol{p}_{p T}\right|^{2} / W^{2}$ and (mass) $)^{2} / W^{2}$ and are thus power-suppressed in the DIS limit [cf. Eq. (4.13)]. This suggests that our collinear LF calculation is safe in the DIS limit.

In addition to the kinematic off-shell effects discussed so far, nuclear binding causes dynamical modifications of the structure of the nucleon, which manifest themselves, e.g., in the suppression of the nuclear structure functions at $x>0.3$ compared to the sum of the corresponding nucleon structure functions (EMC effect). Theoretical analysis shows that to first order in the nuclear binding these modifications are proportional to the LF energy off-shellness of the nuclear configurations (or the nucleon virtuality in the virtual nucleon formulation), which in turn is proportional to the nonrelativistic kinetic energy of the nucleons $[1,60]$. The modifications are therefore much smaller in the deuteron than in heavy nuclei. Simple scaling arguments suggest that in average configurations in the deuteron the EMC-like modifications should be at the level of $\sim 3-4 \%$. They are reduced further when selecting configurations with proton recoil momenta less than the typical nucleon momentum in the deuteron (the median momentum is $\sim 70 \mathrm{MeV}$; see Fig. 17 and Appendix A). The modifications can be eliminated entirely by performing on-shell extrapolation in the recoil momentum, which effectively turns the deuteron into a free $p n$ system (see Sec. IV G).

\section{Spectral function}

The IA for the deuteron tensor in tagged DIS, Eq. (4.9), is conveniently expressed in terms of the deuteron spectral function, defined as

$$
S_{d}\left(\alpha_{p}, \boldsymbol{p}_{p T}\right) \equiv \frac{\left|\Psi_{d}\left(\alpha_{p}, \boldsymbol{p}_{p T}\right)\right|^{2}}{2-\alpha_{p}} .
$$

It is a function of the LF momentum variables of the recoil proton and satisfies the sum rules

$$
\begin{aligned}
& \int_{0}^{2} \frac{d \alpha_{p}}{\alpha_{p}} \int d^{2} p_{p T} S_{d}\left(\alpha_{p}, \boldsymbol{p}_{p T}\right) \\
& =\int \frac{d \alpha_{p} d^{2} p_{T R}}{\alpha_{p}\left(2-\alpha_{p}\right)}\left|\Psi_{d}\left(\alpha_{p}, \boldsymbol{p}_{p T}\right)\right|^{2}=1, \\
& \int_{0}^{2} \frac{d \alpha_{p}}{\alpha_{p}} \int d^{2} p_{p T}\left(2-\alpha_{p}\right) S_{d}\left(\alpha_{p}, \boldsymbol{p}_{p T}\right) \\
& =\int \frac{d \alpha_{p} d^{2} p_{p T}}{\alpha_{p}\left(2-\alpha_{p}\right)}\left(2-\alpha_{p}\right)\left|\Psi_{d}\left(\alpha_{p}, \boldsymbol{p}_{p T}\right)\right|^{2} \\
& =\int \frac{d \alpha_{p} d^{2} p_{p T}}{\alpha_{p}\left(2-\alpha_{p}\right)} \alpha_{p}\left|\Psi_{d}\left(\alpha_{p}, \boldsymbol{p}_{p T}\right)\right|^{2} \\
& =\int \frac{d \alpha_{p} d^{2} p_{p T}}{\alpha_{p}\left(2-\alpha_{p}\right)}\left|\Psi_{d}\left(\alpha_{p}, \boldsymbol{p}_{p T}\right)\right|^{2}=1 .
\end{aligned}
$$

The first sum rule, Eq. (4.21), follows from the normalization condition of the deuteron LF wave function, Eq. (3.14), and reflects the total number of nucleons in the bound state (nucleon number sum rule). The second sum rule, Eq. (4.22), follows from the symmetry of the two-body LF wave function in the transverse rest frame, Eq. (3.16), and expresses the conservation of the LF plus momentum (momentum sum rule). The physical implications of these sum rules will be explained in the following. In terms of the spectral function the IA result for the tagged structure functions, Eqs. (4.18) and (4.19), are now expressed as

$$
\begin{aligned}
& F_{2 d}\left(x, Q^{2} ; \alpha_{p}, p_{p T}\right)=S_{d}\left(\alpha_{p}, \boldsymbol{p}_{p T}\right) F_{2 n}\left(\tilde{x}, Q^{2}\right), \\
& F_{L d}\left(x, Q^{2} ; \alpha_{p}, p_{p T}\right)=\frac{2 S_{d}\left(\alpha_{p}, \boldsymbol{p}_{p T}\right)}{2-\alpha_{p}} F_{L n}\left(\tilde{x}, Q^{2}\right) .
\end{aligned}
$$

It is instructive to consider the integral of the tagged deuteron structure function over the recoil momentum

$$
F_{2 d}^{\mathrm{int}}\left(x, Q^{2}\right) \equiv \int_{0}^{2-x} \frac{d \alpha_{p}}{\alpha_{p}} \int d^{2} p_{p T} F_{2 d}\left(x, Q^{2} ; \alpha_{p}, p_{p T}\right)
$$

The restriction $\alpha_{p}<2-x$ results because the recoil proton plus momentum cannot exceed the total plus momentum of the DIS final state. Notice that this integral over the LF variables corresponds to the integral over the invariant recoil momentum phase space, Eq. (2.45). With the IA expression Eq. (4.23) the 
integrated structure function becomes

$$
\begin{aligned}
F_{2 d}^{\text {int }}\left(x, Q^{2}\right) \\
\equiv \int_{0}^{2-x} \frac{d \alpha_{p}}{\alpha_{p}} \int d^{2} p_{p T} S_{d}\left(\alpha_{p}, \boldsymbol{p}_{p T}\right) F_{2 n}\left(\tilde{x}, Q^{2}\right) \\
=\int_{0}^{2-x} \frac{d \alpha_{p}}{\alpha_{p}\left(2-\alpha_{p}\right)} \int d^{2} p_{p T}\left|\Psi_{d}\left(\alpha_{p}, \boldsymbol{p}_{p T}\right)\right|^{2} F_{2 n}\left(\tilde{x}, Q^{2}\right) \\
\quad\left[\widetilde{x}=x /\left(2-\alpha_{p}\right)\right] .
\end{aligned}
$$

Equation (4.28) has several interesting properties. First, using the symmetry of the LF wave function, Eq. (3.16), the integral can equivalently be expressed as an integral over the active neutron fraction $\alpha_{n}=2-\alpha_{p}$, whereupon it takes the form of a standard partonic convolution formula,

$$
\begin{aligned}
F_{2 d}^{\mathrm{int}}\left(x, Q^{2}\right)= & \int_{x}^{2} \frac{d \alpha_{n}}{\alpha_{n}\left(2-\alpha_{n}\right)} \int d^{2} p_{n T}\left|\Psi_{d}\left(\alpha_{n}, \boldsymbol{p}_{n T}\right)\right|^{2} \\
& \times F_{2 n}\left(\widetilde{x}, Q^{2}\right) \quad\left(\widetilde{x} \equiv x / \alpha_{n}\right) .
\end{aligned}
$$

Second, using the momentum sum rule for the spectral function, Eq. (4.22), and changing the order of the integrations, one easily shows that

$$
\int_{0}^{2} d x F_{2 d}^{\mathrm{int}}\left(x, Q^{2}\right)=\int_{0}^{1} d \widetilde{x} F_{2 n}\left(\widetilde{x}, Q^{2}\right) .
$$

A similar formula applies to the case of tagged neutron and active proton. Together, they imply that the LF momentum sum rule for the deuteron is satisfied exactly in the IA if one adds the contributions from proton and neutron tagging, i.e., from scattering on the active neutron and proton,

$$
\begin{aligned}
& \int_{0}^{2} d x\left[F_{2 d}^{\mathrm{int}}(p \text { tagged })+F_{2 d}^{\mathrm{int}}(n \text { tagged })\right]\left(x, Q^{2}\right) \\
& \quad=\int_{0}^{1} d \widetilde{x}\left[F_{2 n}+F_{2 p}\right]\left(\widetilde{x}, Q^{2}\right) .
\end{aligned}
$$

Third, for nonexceptional values of $x$ the integral over $\alpha_{p}$ in Eq. (4.28) is dominated by the region $\alpha_{p} \sim 1$, so that one can neglect the variation of $\tilde{x}=x /\left(2-\alpha_{p}\right)$ under the integral and evaluate the structure function at $\alpha_{p}=1$ (peaking approximation),

$$
\begin{aligned}
F_{2 d}^{\mathrm{int}}\left(x, Q^{2}\right) & \approx F_{2 n}\left(x, Q^{2}\right) \int_{0}^{2} \frac{d \alpha_{p}}{\alpha_{p}} \int d^{2} p_{p T} S_{d}\left(\alpha_{p}, \boldsymbol{p}_{p T}\right) \\
& =F_{2 n}\left(x, Q^{2}\right)
\end{aligned}
$$

In the second step we have used the number sum rule for the spectral function, Eq. (4.21). Again a similar formula applies to the case of tagged neutron and active proton. Thus the sum of proton-tagged and neutron-tagged deuteron structure functions in the peaking approximation reduces to the sum of the free neutron and proton structure functions, as it should be.

Some comments are in order regarding our definition of the spectral function Eq. (4.20). In the IA for a complex nucleus $(A>2)$ the spectral function describes the probability for removing a nucleon, leaving the $A-1$ remnant system $R$ in a state with given momentum $\boldsymbol{p}_{R}$ and total energy $E_{R}$, which includes the energy of the excitation and/or internal motion of the system. In the IA for the deuteron $(A=2)$, assuming that it can be described as a $p n$ system (neglecting $N N \pi$ and $\Delta \Delta$ components), the recoiling system is a single nucleon, and its energy is fixed by the energy-momentum relation (there is no excitation or internal motion), so that the spectral function depends on the momentum variables only. In fact, the proton-tagged spectral function defined by Eq. (4.20) is related in a simple way to the neutron LF momentum density in the deuteron [11], cf. Eq. (3.16),

$$
\begin{aligned}
S_{d}\left(\alpha_{p}, \boldsymbol{p}_{p T}\right)= & \frac{\alpha_{p}}{\alpha_{n}} \rho_{d}\left(\alpha_{n}, \boldsymbol{p}_{n T}\right), \\
\rho_{d}\left(\alpha_{n}, \boldsymbol{p}_{n T}\right) \equiv & \frac{\left|\Psi_{d}\left(\alpha_{n}, \boldsymbol{p}_{n T}\right)\right|^{2}}{2-\alpha_{n}}=\frac{\left|\Psi_{d}\left(\alpha_{p}, \boldsymbol{p}_{p T}\right)\right|^{2}}{\alpha_{p}} \\
& \left(\alpha_{n}=2-\alpha_{p}, \boldsymbol{p}_{n T}=-\boldsymbol{p}_{p T}\right) .
\end{aligned}
$$

The density is regarded as a function of the neutron LF momentum variables and satisfies the normalization condition

$$
\int \frac{d \alpha_{n}}{\alpha_{n}} \int d^{2} p_{n T} \rho_{d}\left(\alpha_{n}, \boldsymbol{p}_{n T}\right)=1 .
$$

In this sense we could express the IA result (and the distortion effects due to FSI considered below) as well in terms of the active neutron density. We choose to express them in terms of the spectral function Eq. (4.20), as this function depends on the observable recoil proton momentum.

\section{E. Spin degrees of freedom}

It is straightforward to include the deuteron and nucleon spin degrees of freedom in the LF IA. The deuteron tensor in the IA, Eq. (4.4), now becomes

$$
\begin{aligned}
W_{d}^{\mu \nu}\left(p_{d}, q ; p_{p}\right)= & \frac{1}{3} \sum_{\lambda_{d}} \sum_{\sigma_{p}} \sum_{\sigma_{n 1}, \sigma_{n 2}}\left(\frac{p_{d}^{+}}{p_{n}^{+}}\right)^{2}(2 \pi)^{3} \Psi_{d}^{*}\left(\alpha_{p}, \boldsymbol{p}_{p T} ; \sigma_{p}, \sigma_{n 2} \mid \lambda_{d}\right) \Psi_{d}\left(\alpha_{p}, \boldsymbol{p}_{p T} ; \sigma_{p}, \sigma_{n 1} \mid \lambda_{d}\right) \\
& \times(4 \pi)^{-1} \sum_{X}(2 \pi)^{4} \delta^{(4)}\left(q+p_{d}-p_{p}-p_{X}\right)\left\langle n, p_{n}, \sigma_{n 2}\left|\hat{J}^{\mu}(0)\right| X\right\rangle\left\langle X\left|\hat{J}^{\nu}(0)\right| n, p_{n}, \sigma_{n 1}\right\rangle \\
& \left(p_{n}^{+}=p_{d}^{+}-p_{p}^{+}, \boldsymbol{p}_{n T}=-\boldsymbol{p}_{p T}\right),
\end{aligned}
$$

where we average over the initial deuteron LF helicity $\lambda_{d}$, sum over the final proton LF helicity $\sigma_{p}$, and sum over the LF helicities of the intermediate neutron states, $\sigma_{n 1}$ and $\sigma_{n 2}$. Note that a priori the neutrons in the two current matrix elements have different spin quantum numbers, which need to be summed over independently. A simplification arises from the fact that the hadronic 
tensor for inclusive scattering on the neutron is diagonal in the neutron LF helicity,

$$
\begin{aligned}
W_{n}^{\mu \nu}\left(p_{n}, \widetilde{q} ; \sigma_{n 2}, \sigma_{n 1}\right) & \equiv(4 \pi)^{-1} \sum_{X}(2 \pi)^{4} \delta^{(4)}\left(\widetilde{q}+p_{n}-p_{X}\right)\left\langle n, p_{n}, \sigma_{n 2}\left|\hat{J}^{\mu}(0)\right| X\right\rangle\left\langle X\left|\hat{J}^{v}(0)\right| n, p_{n}, \sigma_{n 1}\right\rangle \\
& =\delta\left(\sigma_{n 2}, \sigma_{n 1}\right) \times \text { function }\left(\sigma_{n 1}\right) .
\end{aligned}
$$

Off-diagonal elements would imply a transverse spin dependence of the inclusive neutron cross section, which is forbidden by the combination of $P$ and $T$ invariance and the hermiticity of the electromagnetic current operator (Christ-Lee theorem) $[61,62]$. Moreover, we retain only the unpolarized part of the neutron tensor, which is independent of the diagonal spin variable $\sigma_{n 1}$ and involves the unpolarized neutron structure functions, Eq. (4.14). The spectral function can then be computed as in the spinless case, and we obtain

$$
S_{d}\left(\alpha_{p}, \boldsymbol{p}_{p T}\right) \equiv \frac{1}{3} \sum_{\lambda_{d}} \sum_{\sigma_{p}, \sigma_{n}} \frac{\left|\Psi_{d}\left(\alpha_{p}, \boldsymbol{p}_{p T} ; \sigma_{p}, \sigma_{n} \mid \lambda_{d}\right)\right|^{2}}{2-\alpha_{p}},
$$

which generalizes the spinless result Eq. (4.20).

\section{F. Nonrelativistic approximation}

We now derive the nonrelativistic approximation to the LF spectral function Eq. (4.37), using the nonrelativistic approximation to the deuteron LF wave function, Eq. (3.37), and the corresponding approximation to the LF flux factors. This exercise leads to a practical formula for evaluating the LF spectral function, and explains the correspondence between the LF and nonrelativistic formulation at proton momenta $\left|\boldsymbol{p}_{p}\right|^{2} \ll M_{N}^{2}$. We consider the function

$$
\frac{S_{d}\left(\alpha_{p}, \boldsymbol{p}_{p T}\right)}{2-\alpha_{p}}=\frac{1}{3} \sum_{\lambda_{d}} \sum_{\sigma_{p}, \sigma_{n}} \frac{\left|\Psi_{d}\left(\alpha_{p}, \boldsymbol{p}_{p T} ; \sigma_{p}, \sigma_{n} \mid \lambda_{d}\right)\right|^{2}}{\left(2-\alpha_{p}\right)^{2}} .
$$

in the deuteron rest frame and expand in powers of the proton momentum, $\left|\boldsymbol{p}_{p}\right| / M_{N}$ and $p_{p}^{z} / M_{N}$, cf. Sec. IIIC and Eq. (3.27). The expansion of the squared wave function is given by Eq. (3.29) (the spin rotations drop out),

$$
\begin{aligned}
& \left|\Psi_{d}\left(\alpha_{p}, \boldsymbol{p}_{p T} ; \sigma_{p}, \sigma_{n} \mid \lambda_{d}\right)\right|^{2} \\
& \quad=M_{N}\left(1-\frac{2 p^{z}}{M_{N}}\right)\left|\widetilde{\Psi}_{d}\left(\boldsymbol{p}_{p}, \sigma_{p}, \sigma_{n}\right)\right|^{2}+O\left(\left|\boldsymbol{p}_{p}\right|^{2} / M_{N}^{2}\right)
\end{aligned}
$$

the expansion of the flux factor in Eq. (4.38) is

$$
\frac{1}{\left(2-\alpha_{p}\right)^{2}}=1+\frac{2 p_{p}^{z}}{M_{N}}+O\left(\left|\boldsymbol{p}_{p}\right|^{2} / M_{N}^{2}\right),
$$

and Eq. (4.38) becomes

$$
\begin{aligned}
& \frac{S_{d}\left(\alpha_{p}, \boldsymbol{p}_{p T}\right)}{2-\alpha_{p}} \\
& =M_{N} \frac{1}{3} \sum_{\lambda_{d}} \sum_{\sigma_{p}, \sigma_{n}}\left|\widetilde{\Psi}_{d}\left(\boldsymbol{p}_{p}, \sigma_{p}, \sigma_{n}\right)\right|^{2}+O\left(\left|\boldsymbol{p}_{p}\right|^{2} / M_{N}^{2}\right)
\end{aligned}
$$

$$
\begin{aligned}
& =M_{N} \rho_{d}\left(\boldsymbol{p}_{p}, \boldsymbol{p}_{p}\right)+O\left(\left|\boldsymbol{p}_{p}\right|^{2} / M_{N}^{2}\right) \\
& =M_{N}\left[U^{2}\left(p_{p}\right)+W^{2}\left(p_{p}\right)\right]+O\left(\left|\boldsymbol{p}_{p}\right|^{2} / M_{N}^{2}\right) .
\end{aligned}
$$

Here $\rho_{d}\left(\boldsymbol{p}_{p}, \boldsymbol{p}_{p}\right)$ is the diagonal, spin-averaged, nonrelativistic momentum density in the deuteron, which involves the sum of $S$ - and $D$-wave densities and is given in Eq. (A10). Notice that both the LF wave function and the flux factor involve corrections linear in $p_{p}^{z}$, which refer explicitly to the LF direction and break rotational symmetry. In the function Eq. (4.43), however, the linear corrections cancel, and the first corrections are quadratic in the recoil momentum components. This shows that rotational invariance is effectively restored in the LF formulation at small recoil momenta. It implies that the results of the LF IA are numerically close to those of the conventional nonrelativistic IA at recoil momenta $\left|\boldsymbol{p}_{p}\right| \ll M_{N}$. Equation (4.43) also ensures proper analyticity of the LF expressions in the invariant momentum transfer $t^{\prime}$ (see Sec. IV G). Finally, it can be used for numerical evaluation of the LF spectral function at small proton momenta $\left|\boldsymbol{p}_{p}\right| \lesssim 300 \mathrm{MeV}$.

\section{G. Analytic properties}

We now want to study the analytic properties of the IA spectral function in the invariant momentum transfer $t^{\prime}$. For this purpose it is natural to use as independent variables $t^{\prime}$ and the proton LF fraction $\alpha_{p}$; the relation of these variables to $\alpha_{p}$ and $\boldsymbol{p}_{p T}$ is given by Eq. (2.55). The analytic properties of the spectral function are governed by the nucleon pole of the deuteron LF wave function, Eq. (3.26), which occurs in the $S$ wave. The invariant mass difference in Eq. (3.26) is expressed in terms of $\alpha_{p}$ and $t^{\prime}$ as

$$
s_{p n}-M_{d}^{2}=\frac{-2 t^{\prime}}{2-\alpha_{p}} .
$$

One sees that the LF spectral function Eq. (4.20) in the limit $t^{\prime} \rightarrow 0$ at fixed $\alpha_{p}$ behaves as

$$
\begin{gathered}
S_{d}\left(\alpha_{p}, \boldsymbol{p}_{p T}\right) \sim \frac{R}{\left(t^{\prime}\right)^{2}}+\operatorname{terms} O\left(t^{\prime-1}\right) \quad\left(t^{\prime} \rightarrow 0, \alpha_{p} \text { fixed }\right), \\
R \equiv R\left(\alpha_{p}\right)=4 M_{N} \Gamma^{2}\left(2-\alpha_{p}\right) .
\end{gathered}
$$

The spectral function has a pole at $t^{\prime}=0$, whose residue depends on $\alpha_{p}$ and is calculable in terms of the residue of the pole of the three-dimensional deuteron wave function, $\Gamma$. We note that (a) the nucleon pole is a general feature and relies only on rotational invariance and the analytic properties of the rest-frame wave function; (b) the pole occurs in the $S$-wave contribution to the spectral function in Eq. (4.43); the $D$-wave contribution is regular at $t^{\prime}=0$; (c) the pole in the spectral function is reproduced by the relativistically invariant formulation of high-energy scattering on the deuteron 
(Feynman diagrams, virtual nucleon approximation), where it corresponds to "nucleon exchange" between the deuteron and the electromagnetic current; (d) the pole Eq. (4.45) represents the leading singularity in the limit $t^{\prime} \rightarrow$ and is contained in the IA cross section; FSIs modify only subleading singularities in $t^{\prime}$, as was proven in general in Ref. [15] and will be demonstrated explicitly using the specific model of FSIs derived in Sec. VI.

In the limit $t^{\prime} \rightarrow 0$ the invariant mass difference in the deuteron LF wave function tends to zero, Eq. (4.44). This implies that the LF energy off-shellness of the $p n$ system in the IA vanishes [cf. Eqs. (4.6) and (4.7)],

$$
\begin{aligned}
p_{d}^{+}\left(\widetilde{q}^{-}-q^{-}\right) & =p_{d}^{+}\left(p_{d}^{-}-p_{n}^{-}-p_{p}^{-}\right) \\
& =-\left(s_{p n}-4 M_{N}^{2}\right) \rightarrow 0 .
\end{aligned}
$$

The kinematic shift in the four-momentum transfer, $\widetilde{q}-q$, Eq. (4.7), therefore disappears at the pole, and the IA effectively describes the scattering from a free on-shell neutron.

The analytic properties of the LF spectral function suggest a natural method for extracting the free neutron structure functions from proton-tagged DIS measurements on the deuteron. One measures the proton-tagged structure function at fixed $Q^{2}$ as a function of $x$ and the recoil proton momentum $\left|\boldsymbol{p}_{p}\right|$. One then tabulates the tagged structure function data in $\alpha_{p}$ and $t^{\prime}$, which extends over the physical region $t^{\prime}<t_{\min }^{\prime}$. The free neutron structure function is then obtained by multiplying the tagged structure function data by $\left(t^{\prime}\right)^{2} / R$ (i.e., extracting the pole factor of the spectral function) and extrapolating the resulting data to $t^{\prime} \rightarrow 0$ (on-shell extrapolation). The procedure gives the residue of the tagged structure function at the pole (with the residue of the spectral function removed), which by definition is the free neutron structure function. Nuclear binding and FSI only modify the tagged structure function at $t^{\prime} \neq 0$ but drop out at the pole, so that the procedure is exact in principle. In practice its accuracy is determined by the variation of the tagged structure function in $t^{\prime}$ away from the pole. This question will be addressed with the specific model of FSIs developed in Sec. VI.

The on-shell extrapolation also practically eliminates the $D$-wave contribution to the deuteron spectral function. The $D$ wave does not contain the nucleon pole and is regular at $t^{\prime}=0$. At the same time, because of the angular momentum $L=2$, its wave function is proportional to $\left|\boldsymbol{p}_{p}\right|^{2}$ at small $\left|\boldsymbol{p}_{p}\right|$. Because $t^{\prime}=0$ corresponds to small unphysical momenta $\left|\boldsymbol{p}_{p}\right|^{2}=$ $-a^{2}=-\epsilon_{d} M_{N}$, cf. Eq. (3.25), the $D$-wave contribution to the spectral function is $O\left(a^{4}\right)$ at $t^{\prime}=0$ and thus extremely small.

We evaluate the IA spectral function numerically, using the nonrelativistic approximation Eq. (4.43) and the AV18 deuteron wave function [56]. Figure 7 shows the $t^{\prime}$ dependence of the IA spectral function after extraction of the pole factor $R /\left(t^{\prime}\right)^{2}$. [ $t^{\prime}$ is obtained from the proton rest-frame momentum $\left|\boldsymbol{p}_{p}\right|$ through Eq. (2.53).] One sees that the dependence is smooth over a broad region $\left|t^{\prime}\right| \lesssim 0.1 \mathrm{GeV}^{2}$, suggesting that a polynomial fit would permit accurate extrapolation to $t^{\prime}=0$. (The minimum value $\left|t_{\min }^{\prime}\right|$ is indicated on the graph.) The plot shows the $S$-wave contribution and the sum of $S$ and $D$ waves (total). One sees that the $S$ wave dominates at small $\left|t^{\prime}\right|$, and

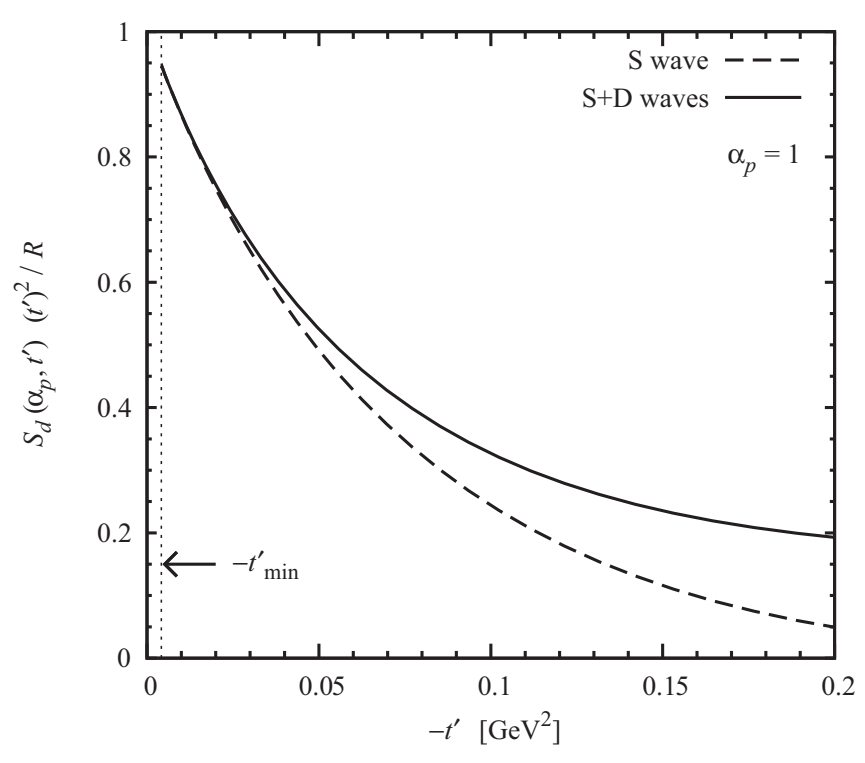

FIG. 7. The deuteron spectral function in the IA, $S_{d}$ [IA], with the pole factor $R /\left(t^{\prime}\right)^{2}$ extracted, as function of $t^{\prime}$, for $\alpha_{p}=1$. The spectral function is evaluated in the nonrelativistic approximation Eq. (4.43) using the AV18 deuteron wave function [56]. Dashed line: $S$-wave contribution only. Solid line: Sum of $S$ and $D$ waves.

that the $D$ wave disappears in the limit $t^{\prime} \rightarrow 0$, as expected on general grounds.

\section{FINAL-STATE HADRON DISTRIBUTIONS}

\section{A. Kinematic variables}

FSIs in tagged DIS arise from interactions of the spectator nucleon with "slow" hadrons produced by the DIS process on the active nucleon (rest frame momenta $\left|\boldsymbol{p}_{h}\right| \lesssim 1 \mathrm{GeV}$, or target fragmentation region; see Sec. I). In order to calculate these effects we need to study the properties of the slow hadron distributions in DIS on the nucleon and parametrize them for our purposes. In this section we discuss the kinematic variables characterizing the final-state hadron distributions, the conditional structure functions, and the basic features of experimental distributions.

For the theoretical description of DIS on the nucleon $(N=$ $p, n)$ we use a frame where the nucleon momentum $\boldsymbol{p}_{N}$ and the momentum transfer $\boldsymbol{q}$ are collinear and define the $z$-axis of the coordinate system (cf. Sec. IIC). ${ }^{3}$ In such a frame the LF components of the nucleon four-momentum $p_{N}$ and the four-momentum transfer $q$ are

$$
\left.\begin{array}{lll}
p_{N}^{+}>0(\text { arbitrary }), & p_{N}^{-}=\frac{M_{N}^{2}}{p_{N}^{+}}, & \boldsymbol{p}_{N T}=0 \\
q^{+}=-\xi p_{N}^{+}, & q^{-}=\frac{Q^{2}}{\xi p_{N}^{+}}, & \boldsymbol{q}_{T}=0
\end{array}\right\},
$$

${ }^{3}$ In the calculation of FSIs in tagged DIS on the deuteron below we shall neglect the effect of the active nucleon's transverse momentum on the final-state hadron spectrum, so that the $z$ axis axis of the virtual photon-nucleon frame coincides with that of the virtual photondeuteron frame. 


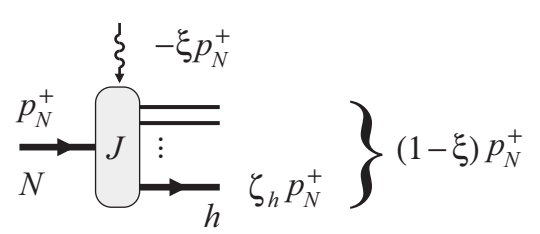

FIG. 8. Current matrix element in DIS on the nucleon with an identified hadron $h$ in the nucleon fragmentation region, $e N \rightarrow$ $e^{\prime}+h+X^{\prime}$. The LF plus momenta of the final state are expressed as fractions of $p_{N}^{+}$.

where $p_{N}^{+}$is arbitrary and defines the particular frame, and the variable $\xi$ is determined by

$$
\begin{aligned}
\xi & =\frac{2 x}{1+\sqrt{1+4 M_{N}^{2} x^{2} / Q^{2}}} \\
& =x+O\left(M_{N}^{2} / Q^{2}\right) \quad\left(Q^{2} \gg M_{N}^{2}\right) .
\end{aligned}
$$

With this choice of components the momentum transfer vector $\boldsymbol{q}$ points in the negative $z$ direction, $2 q^{z}=q^{+}-q^{-}<0$.

An identified hadron $h$ in the DIS final state is characterized by its LF momentum $p_{h}^{+} \equiv \zeta_{h} p_{N}^{+}$and transverse momentum $\boldsymbol{p}_{h T}$ (see Fig. 8). Because the hadron LF momentum cannot exceed the total LF momentum of the DIS final state, $p_{N}^{+}+$ $q^{+}=(1-\xi) p_{N}^{+}$, the hadron fraction $\zeta_{h}$ is restricted to

$$
0<\zeta_{h}<1-\xi
$$

In particular, values $\zeta_{h} \sim 1$ become kinematically accessible only for $x \sim \xi \ll 1$.

It is instructive to consider the hadron momentum distribution in the nucleon rest frame in terms of the ordinary momentum variables. The connection with the LF momentum distribution can be established easily, because the nucleon rest frame is a special collinear frame with $p_{N}^{+}=M_{N}$. The fraction $\zeta_{h}$ is related to the $z$-component of the hadron rest frame momentum $p_{h}^{z}$ by

$$
\begin{aligned}
& \zeta_{h}=\frac{\sqrt{\left(p_{h}^{z}\right)^{2}+\left|\boldsymbol{p}_{h T}\right|^{2}+M_{h}^{2}}+p_{h}^{z}}{M_{N}}, \\
& p_{h}^{z}=-\frac{\left|\boldsymbol{p}_{h T}\right|^{2}+M_{h}^{2}-\zeta_{h}^{2} M_{N}^{2}}{2 \zeta_{h} M_{N}} .
\end{aligned}
$$

One observes the following: (a) If $M_{h} \geqslant M_{N}$ (e.g., if the identified hadron is a nucleon) the hadron $z$-momentum is negative, i.e., along the direction of the $\boldsymbol{q}$-vector. Such hadrons always go "forward" in the rest frame, meaning in the direction of the $\boldsymbol{q}$-vector. The momentum distribution is a cone opening in the negative $z$ direction. (b) If $M_{h}<M_{N}$ (e.g., if the identified hadron is a pion) the hadron $z$-momentum can be positive for sufficiently small $\left|\boldsymbol{p}_{h T}\right|$, i.e., opposite to the direction of the $\boldsymbol{q}$-vector. Such hadrons can go "backwards" in the nucleon rest frame.

Figure 9 shows the momentum distribution of nucleons $\left(M_{h}=M_{N}\right)$ in the nucleon fragmentation region for fixed values of $\zeta_{h}$. One sees that small longitudinal momenta $p_{h}^{z} \rightarrow 0$ correspond to LF fractions $\zeta_{h} \rightarrow 1$, and that the cones are shifted to larger longitudinal momenta as $\zeta_{h}$ deviates from

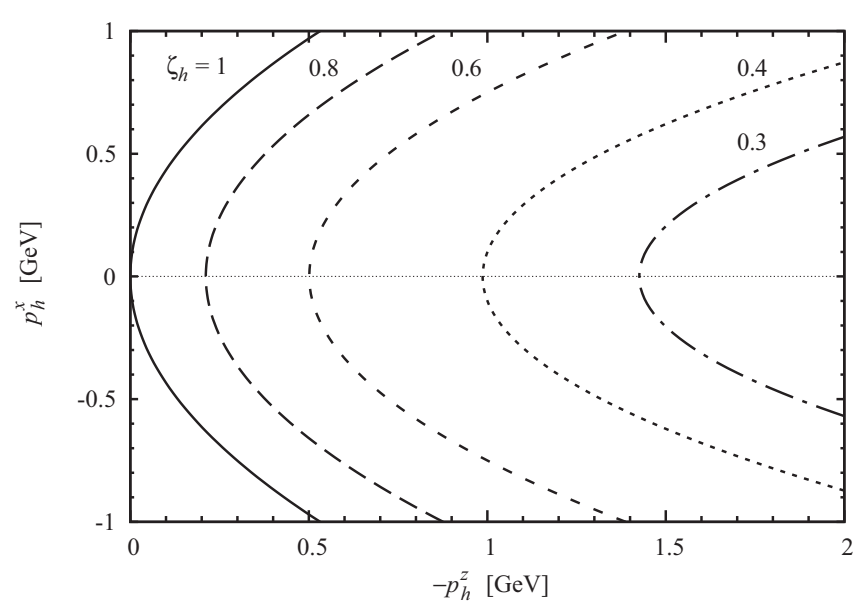

FIG. 9. Momentum distributions of nucleons $\left(M_{h}=M_{N}\right)$ in the nucleon fragmentation region in DIS, $e N \rightarrow e^{\prime}+h+X$. The contours show the allowed values of $p_{h}^{z}$ and $p_{h}^{x}$ (with $p_{h}^{y}=0$, so that $\left.\left|\boldsymbol{p}_{h T}\right|=\left|p_{h}^{x}\right|\right)$ for given constant values of $\zeta_{h}$. The contours thus describe the intersection of the allowed three-momentum cones with the transverse $x$ plane.

unity. Note that $\zeta_{h}$ is kinematically restricted by Eq. (5.3). Figure 10 shows the minimal three-momentum of nucleons in the nucleon fragmentation region as a function of $\xi$. The minimal value of the three-momentum is attained for $\boldsymbol{p}_{h T}=0$ [see Eq. (5.5) and Fig. 9] and is given by

$$
\left|\boldsymbol{p}_{h}\right|(\min )=\frac{\xi(1-\xi / 2) M_{N}}{1-\xi} \quad(h=N) .
$$

One sees that nucleons with $\left|\boldsymbol{p}_{h}\right| \lesssim 1 \mathrm{GeV}^{2}$ appear only if $x \sim \xi \ll 1$. Note that Eq. (5.6) gives only the kinematic limit,

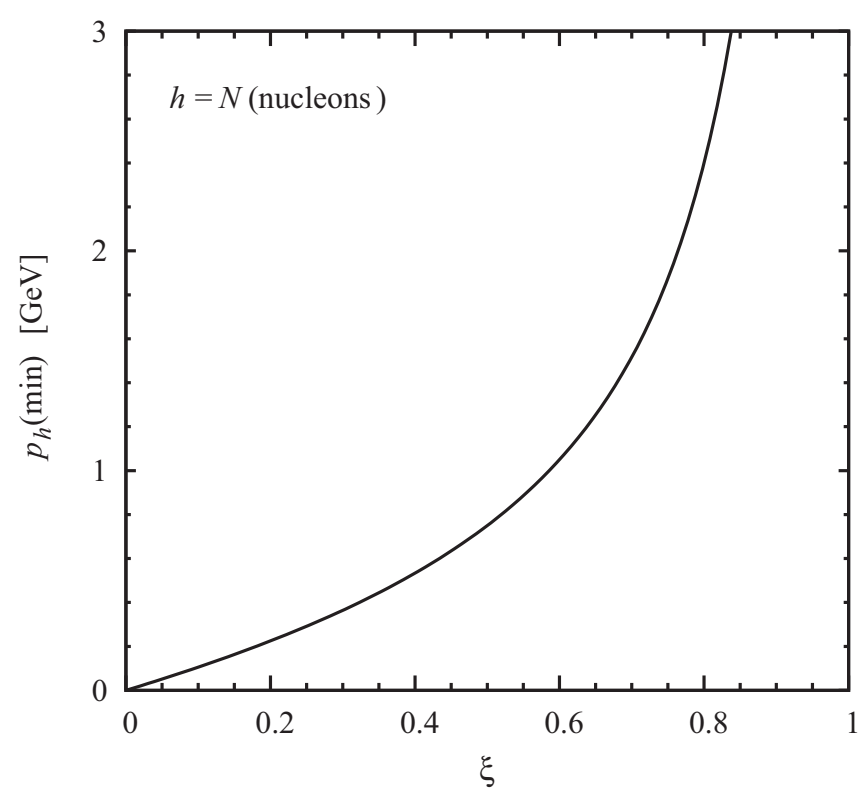

FIG. 10. Minimal three-momentum $\left|\boldsymbol{p}_{h}\right|(\mathrm{min})$ of nucleons $\left(M_{h}=\right.$ $M_{N}$ ) in the nucleon fragmentation region in DIS, Eq. (5.6), as a function of the variable $\xi=x+O\left(M_{N}^{2} / Q^{2}\right)$, Eq. (5.2). 
and that the average values of the nucleon momenta in the fragmentation region are substantially larger, because the phase space opens up with the transverse momentum.

The LF variables $\zeta_{h}$ and $\boldsymbol{p}_{h T}$ can be related to other variables used to characterize experimental hadron distributions in DIS. One commonly used variable is the fraction of the rest-frame energy transfer carried by the hadron,

$$
z_{h} \equiv \frac{E_{h}}{v}, \quad v=\frac{Q^{2}}{2 M_{N} x}=\frac{Q^{2}}{2 M_{N} \xi}\left(1-\frac{\xi^{2} M_{N}^{2}}{Q^{2}}\right) .
$$

Using Eq. (5.4) and setting

$$
p_{h}^{z}=-\sqrt{E_{h}^{2}-M_{h T}^{2}}, \quad M_{h T}^{2} \equiv\left|\boldsymbol{p}_{h T}\right|^{2}+M_{h}^{2},
$$

one obtains

$$
\zeta_{h}=\frac{z v-\sqrt{(z v)^{2}-M_{h T}^{2}}}{M_{N}} \approx \frac{M_{h T}^{2}}{2 z v M_{N}}=\frac{x M_{h T}^{2}}{z Q^{2}} \quad\left(z v \gg M_{h T}\right),
$$

$z_{h}=\frac{\zeta_{h}^{2} M_{N}^{2}+M_{h T}^{2}}{2 \zeta_{h} M_{N} v}$

One sees that LF fractions $\zeta_{h}=O(1)$ correspond to parametrically small energy fractions $z=O\left(M_{h T} / v\right) \ll 1$ in the nucleon rest frame (slow hadrons). Another commonly used variable is the hadron's longitudinal momentum in the CM frame of the virtual photon-nucleon collision, in which $\boldsymbol{q}+$ $\boldsymbol{p}_{N}=0$. It is usually expressed in terms of the Feynman scaling variable $^{4}$

$$
x_{\mathrm{F}} \equiv-\frac{p_{h}^{z}}{p_{h, \text { max }}^{z}} .
$$

The connection with the LF variables is established by noting that the $\mathrm{CM}$ frame is the special collinear frame with

$$
p_{N}^{+}=\sqrt{\frac{Q^{2}+\xi M_{N}^{2}}{\xi(1-\xi)}} .
$$

The hadron longitudinal $\mathrm{CM}$ momentum is

$$
p_{h}^{z}=\frac{p_{h}^{+}+p_{h}^{-}}{2}=\frac{\zeta_{h}^{2}\left(p_{N}^{+}\right)^{2}-M_{h T}^{2}}{2 \zeta_{h} p_{N}^{+}} .
$$

The maximum (positive) value is attained for $\zeta_{h}=1-\xi$ and $\boldsymbol{p}_{h T}=0$,

$$
p_{h, \max }^{z}=\frac{(1-\xi)^{2}\left(p_{N}^{+}\right)^{2}-M_{h}^{2}}{2(1-\xi) p_{N}^{+}} .
$$

The scaling variable is thus obtained as

$x_{\mathrm{F}}=-\frac{(1-\xi)\left[\zeta_{h}^{2}\left(p_{N}^{+}\right)^{2}-M_{h T}^{2}\right]}{\zeta_{h}\left[(1-\xi)^{2}\left(p_{N}^{+}\right)^{2}-M_{h}^{2}\right]} \approx-\frac{\zeta_{h}}{1-\xi} \quad\left(\zeta_{h} p_{N}^{+} \gg M_{h T}\right)$.

\footnotetext{
${ }^{4}$ The variable $x_{\mathrm{F}}$ in electroproduction is conventionally defined such that hadrons moving in the virtual photon direction have $x_{\mathrm{F}}>0$, and hadrons moving in the target direction have $x_{\mathrm{F}}<0$. In our convention $q^{z}<0$ and $p_{N}^{z}>0$, hence the minus sign in Eq. (5.11).
}

The latter condition is fulfilled if $Q^{2} \gg M_{h T}^{2}$ (DIS limit) and $\zeta_{h}=O(1)$. One concludes that $-x_{\mathrm{F}}$ in the nucleon fragmentation region (from -1 to approximately -0.5 ) can be identified directly with the normalized hadron LF fraction $\zeta_{h} /(1-\xi)$. We use this relation in our analysis of experimental slow hadron spectra below.

\section{B. Multiplicity distributions}

The hadronic tensor and differential cross section for DIS on the nucleon with an identified final-state hadron $h$ are described by expressions analogous to those for DIS on the deuteron with an identified nucleon in Sec. IIB; see Eqs. (2.19) and (2.31). The hadronic tensor is parametrized by conditional nucleon structure functions

$$
F_{2 N, h}\left(x, Q^{2} ; \zeta_{h}, \boldsymbol{p}_{h T}\right), \text { etc., }
$$

which depend on the identified hadron's LF momentum fraction $\zeta_{h}$ and transverse momentum $\boldsymbol{p}_{h T}$. It is convenient to extract the inclusive structure functions and write the conditional structure functions in the form

$$
F_{2 N, h}\left(x, Q^{2} ; \zeta_{h}, \boldsymbol{p}_{h T}\right)=F_{2 N}\left(x, Q^{2}\right) D_{h}\left(x, Q^{2} ; \zeta_{h}, \boldsymbol{p}_{h T}\right), \text { etc. }
$$

The function $D_{h}$ describes the normalized differential multiplicity distribution of the hadron $h$, i.e., the differential number of hadrons $d N_{h}$ per DIS event observed in a phase space element $d \Gamma_{h}$ :

$$
\frac{d N_{h}}{N_{\text {incl }}}=D_{h} d \Gamma_{h}, \quad d \Gamma_{h}=\frac{d \zeta_{h} d^{2} p_{h T}}{2(2 \pi)^{3} \zeta_{h}} .
$$

As such it can be directly extracted from the experimental multiplicity distributions. In particular, the $p_{h T}$-integrated LF momentum distribution of the hadron is

$$
\frac{1}{N_{\text {incl }}} \frac{d N_{h}}{d \zeta_{h}}=\frac{1}{2(2 \pi)^{3} \zeta_{h}} \int d^{2} p_{h T} D_{h} .
$$

It can be identified with the $x_{\mathrm{F}}$ distribution in the nucleon fragmentation region, cf. Eq. (5.15). Note the factor $1 / \zeta_{h}$ on the right-hand side, which results from the definition of the invariant phase space element Eq. (5.18).

\section{Experimental distributions}

Measurements of hadron multiplicity distributions in the target fragmentation region in DIS on the nucleon have been reported by several fixed-target experiments using electron beams (Cornell Synchrotron [63]) and muon beams (CERN EMC [64-66], FNAL E665 [25]), as well as at the HERA electron-proton collider [67-70]. Slow hadron distributions were also measured in neutrino-proton DIS experiments [7173]. While the kinematic coverage is far from complete, these data roughly cover the $x$ region of interest for our study and allow us to infer the basic features of the multiplicity distributions. Unfortunately many data are not separated according to hadron species, as few dedicated studies of the target fragmentation region have been performed so far. We now briefly review the main features of the data and their theoretical interpretation. 
The multiplicity distributions of hadrons with $x_{\mathrm{F}} \lesssim-0.2$ are approximately independent of $Q^{2}$ for fixed $x$. Scaling of the distributions is observed in all quoted electron and muon experiments, covering the valence region $x>0.2$ [63], the region $x \lesssim 0.1$ [25,64-66], and the small- $x$ region $x<$ $10^{-2}$ [67-70]. This behavior is consistent with theoretical expectations based on QCD factorization of the conditional DIS cross sections in the target fragmentation region [30,31]. The multiplicity distributions in the target fragmentation region show only weak variation with $x$ in the region $x \lesssim 0.1$. This indicates that the hadronization of the target remnant is largely independent of the dynamics producing the parton distributions in the nucleon in this region of $x$ (sea quarks, gluons).

The $x_{\mathrm{F}}$ distributions of protons (integrated over transverse momentum) in DIS on the proton are approximately flat for $x_{\mathrm{F}}<-0.3$. A value of $\left(1 / N_{\text {incl }}\right) d N_{p} / d x_{\mathrm{F}} \sim 0.5-0.6$ at $x_{\mathrm{F}}=$ $(-0.7,-0.3)$ was measured by EMC at $\langle x\rangle=0.1$ [65]. A value $\left(1 / N_{\text {incl }}\right) d N_{p} / d x_{\mathrm{F}} \sim 0.4$ at $x_{\mathrm{F}}=(-0.8,-0.4)$ was obtained by the HERA experiments at $x \lesssim 0.01[67,70]$. (At larger negative $x_{\mathrm{F}}$ diffraction gives rise to a distinct contribution to the proton spectrum at HERA; this mechanism is marginal in the kinematic region considered here.) The $x_{\mathrm{F}}$ distribution of neutrons measured in DIS on the proton at HERA $[68,69]$ is also flat and has a value of $\left(1 / N_{\text {incl }}\right) d N_{p} / d x_{\mathrm{F}} \sim 0.2$ at $x_{\mathrm{F}}=(-0.8,-0.4)$. The sum of proton and neutron multiplicity distributions is thus $\left(1 / N_{\text {incl }}\right) d N_{p+n} / d x_{\mathrm{F}} \sim 0.6$ at $x_{\mathrm{F}}=$ $(-0.8,-0.4)$. That this value is significantly less than 1 shows that part of the baryon number is transported to smaller $x_{\mathrm{F}}$ and materializes outside the target region. We note that both the flatness of the distributions and the baryon number transport are reproduced by string models of the fragmentation mechanism.

The transverse momentum distributions of protons and neutrons in the target fragmentation region drop steeply with $p_{T, h}$ and can be approximated by Gaussian distributions $\sim \exp \left(-B_{h} p_{h T}^{2}\right)(h=p, n)$, where the slope $B_{h}$ determines the average squared transverse momentum as $\left\langle p_{h T}^{2}\right\rangle=B_{h}^{-1}$. The empirical slope for protons is $B_{p} \sim 4 \mathrm{GeV}^{-2}$ at $x>0.2$ (Cornell) [63] and $B_{p}=6-8 \mathrm{GeV}^{-2}$ at $x<10^{-2}$ (HERA) $[67,70]$. A value of $B_{p} \sim 6 \mathrm{GeV}^{-2}$ was also observed in neutrino DIS at $W^{2}<50 \mathrm{GeV}^{2}$ [71].

The multiplicity distribution of charged pions shows very different behavior from that of protons and neutrons. The $x_{\mathrm{F}}$ distribution of pions are significantly smaller than those of protons at $x_{\mathrm{F}}<-0.3$ but rise strongly at $x_{\mathrm{F}}>-0.3$ [65]. Pion production thus happens mainly in the central region of the DIS process and is governed by other dynamical mechanisms than target fragmentation.

\section{Implications for FSI}

The experimental results described in Sec. V C characterize the slow hadron distributions causing FSI in tagged DIS on the deuteron. We now want to summarize the implications and formulate a simple parametrization of the slow hadron distribution for our subsequent calculations.

The dominant hadrons produced in electron-nucleon DIS at $\zeta_{h}>0.2$ are protons and neutrons emerging from the hadronization of the remnant of the active nucleon. These protons and neutrons can interact with the spectator nucleon with the large $N N$ cross section of $\sim 40 \mathrm{mb}$ at momenta $\left|\boldsymbol{p}_{h}\right| \sim$ 1-2 GeV (see Fig. 9 and Appendix C). We therefore suppose that the dominant FSI in tagged DIS at $x \sim 0.1-0.5$ arises from such protons and neutrons in the target fragmentation region of the active nucleon. FSIs induced by pions could in principle be treated within the same picture but are expected to be small.

If the active nucleon in the deuteron is the proton (i.e., if the neutron is tagged), the multiplicity distributions of slow nucleons (protons plus neutrons) can be inferred directly from the proton DIS data. If the active nucleon is a neutron (proton tagged), we suppose that at $x \sim 0.1$ the distribution of slow nucleons (protons plus neutrons) is approximately the same as in DIS on the proton, because the deep-inelastic process occurs mainly on singlet sea quarks produced by gluon radiation and does not change the flavor structure of the baryon remnant system (we neglect the effect of the flavor asymmetry of sea quarks in this context). Since furthermore the nucleon-nucleon cross section at momenta $\sim$ few $\mathrm{GeV}$ is approximately the same for $p p, p n$, and $n n$ scattering (see Appendix C), the FSI effect is the same for deuteron DIS with active proton or active neutron. These approximations permit a model-independent estimate of FSI effects at $x \sim 0.1$ and will be used in our subsequent calculations. The physical picture of FSIs and the formulas derived in the following are valid also at larger $x(\lesssim 0.5)$, where the scattering primarily occurs mainly on valence quarks; in this region they should be evaluated with a detailed model of the quark flavor dependence of the slow hadron multiplicity distributions.

In our numerical studies of FSI in tagged deuteron DIS we use a simple parametrization of the multiplicity distribution of slow protons and neutrons at $x \sim 0.1, D_{h}(h=p, n)$, which reflects the basic features of the experimental distributions and offers sufficient flexibility to study the dependence of FSIs on the slow hadron distribution. We parametrize the distribution in the form

$$
\begin{aligned}
& D_{h}\left(x, Q^{2} ; \zeta_{h}, p_{h T}\right) \\
& =\left[2(2 \pi)^{3}\right] \zeta_{h} f_{h}\left(\zeta_{h}\right) g_{h}\left(p_{h T}\right) \quad(h=p, n ; x \sim 0.1) .
\end{aligned}
$$

The function $f_{h}\left(\zeta_{h}\right)$ describes the $\zeta_{h}$ distribution and can be identified with the $p_{h T}$-integrated multiplicity distribution Eq. (5.19). We choose it such that

$$
\begin{gathered}
f_{h}\left(\zeta_{h}\right) \sim c_{h}=\text { const } \quad\left(1>\zeta_{h} \gtrsim \zeta_{0}\right), \\
f_{h}\left(\zeta_{h}\right) \rightarrow 0 \quad\left(\zeta_{h} \rightarrow 0\right) .
\end{gathered}
$$

The constant $c_{h}$ can be inferred from the experimental proton/neutron $\zeta_{h}$ (or $x_{\mathrm{F}}$ ) distributions in the "flat" region. For the sum of proton and neutron distributions it is

$$
\sum_{h=p, n} c_{h}=c_{p}+c_{n}=0.6-0.8 .
$$

The cutoff at $\zeta_{h} \rightarrow 0$ limits the distribution to slow hadrons in the nucleon rest frame, which are fully formed inside the deuteron and interact with the spectator with the $N N$ cross section. A value $\zeta_{0} \sim 0.2$ corresponds to rest-frame momenta $\left|\boldsymbol{p}_{h}\right| \lesssim 2 \mathrm{GeV}$ (see Fig. 9). The simplest choice for $f_{h}\left(\zeta_{h}\right)$ is a 
step function

$$
f_{h}\left(\zeta_{h}\right)=c_{h} \Theta\left(\zeta_{h}>\zeta_{0}\right)
$$

the results are not sensitive to the details of the cutoff. The function $g_{h}\left(p_{h T}\right)$ describes the normalized $p_{h T}$ distribution of the protons/neutrons and is modeled by a Gaussian,

$$
g_{h}\left(p_{h T}\right)=\frac{B_{h}}{\pi} \exp \left(-B_{h} p_{h T}^{2}\right), \quad \int d^{2} p_{h T} g_{h}\left(p_{h T}\right)=1,
$$

with an empirical slope

$$
B_{h}=6-8 \mathrm{GeV}^{-2} \quad(h=p, n) .
$$

As explained above, the model distribution is used for the slow protons/neutrons in DIS on either the proton or neutron in the deuteron. It applies to DIS at $x \sim 0.1$; for larger $x$ it should be replaced by a more detailed model that includes flavor dependence and the dynamical effect of $x$ on the $\zeta_{h}$ distribution.

In our treatment of FSIs we describe the interactions of the slow protons/neutrons with the spectator nucleon as onshell rescattering with an effective interaction. Off-shell effects are physically related to effects of the finite hadron formation time and can be modeled as a modification of the on-shell effective interaction and the slow proton/neutron distribution. The on-shell effective interaction (scattering amplitude) can be determined from the $N N$ total and elastic cross section data. The main features of the data and a simple parametrization of the amplitude at incident momenta $\left|\boldsymbol{p}_{h}\right| \lesssim 2 \mathrm{GeV}$ are described in Appendix C.

Little is known about the dependence of the slow hadron spectrum on the nucleon spin, and about the effective polarization of the slow protons/neutrons. In the present study we neglect the dependence of the slow-hadron distribution on the nucleon spin, and regard the slow hadrons as effectively unpolarized. Both effects could be included in the calculation without changes in the theoretical framework. Information about the dependence of the slow hadron spectrum on the nucleon spin could come from measurements of target fragmentation in polarized DIS with a future EIC.

\section{FINAL-STATE INTERACTIONS}

\section{A. FSI and IA currents}

We now proceed to calculate the tagged DIS cross section including FSIs between the spectator nucleon and the slow hadrons (protons/neutrons) emerging from the fragmentation of the active nucleon, in the physical picture described in Secs. I and VD. The calculation is performed in LF quantum mechanics in the collinear frame $\boldsymbol{p}_{d T}=0$ as in Sec. IV B and identifies corrections to the IA current and the deuteron tensor resulting from FSIs.

To properly account for the configurations in which FSIs can and cannot occur, we separate the multihadron states $X$ produced in DIS on the nucleon into two classes:

(a) Multihadron states not containing a slow hadron capable of inducing FSIs, which we denote by $X_{0}$. (b) Multihadron states containing a slow hadron $h$ capable of inducing FSIs, which we denote by $X_{1}$. Their state vectors are of the form

$$
\left|X_{1}\right\rangle=\left|h, X^{\prime}\right\rangle \equiv|h\rangle\left|X^{\prime}\right\rangle,
$$

where $X^{\prime}$ is the product state of the remaining hadrons. The summation over these states is performed as

$$
\sum_{X_{1}} \equiv \int d \Gamma_{h} \sum_{X^{\prime}} .
$$

By construction the classes $X_{0}$ and $X_{1}$ then exhaust all possible multihadron states, and the summation over all states becomes

$$
\sum_{X}=\sum_{X_{0}}+\sum_{X_{1}}
$$

The separation is possible because the average slow hadron multiplicity is $<1$ (cf. Sec. VD), i.e., we can assume that the final state contains zero or one slow hadrons, but not more.

We now consider tagged DIS on the deuteron separately for final states $X_{0}$ and $X_{1}$,

$$
e+d \rightarrow e^{\prime}+p+\left(X_{0} \text { or } X_{1}\right) .
$$

For final states of type $X_{0}$ FSIs cannot occur, and the transition current is identical to that obtained in the IA, Eqs. (4.2) and (4.3),

$$
\left\langle p X_{0}\left|\hat{J}^{\mu}(0)\right| d\right\rangle=\left\langle p X_{0}\left|\hat{J}^{\mu}(0)\right| d\right\rangle[\mathrm{IA}] .
$$

For final states of type $X_{1}$ the transition current is computed by inserting plane-wave nucleon and slow hadron intermediate states into the current matrix element (cf. Fig. 11),

$$
\begin{aligned}
\left\langle p X_{1}\left|\hat{J}^{\mu}(0)\right| d\right\rangle \equiv & \left\langle p h X^{\prime}\left|\hat{J}^{\mu}(0)\right| d\right\rangle \\
= & \int\left[d p_{n}\right] \int\left[d p_{p 1}\right] \int\left[d p_{h 1}\right] \\
& \times\left\langle p, p_{p} ; h, p_{h} \mid p, p_{p 1} ; h, p_{h 1}\right\rangle \\
& \times\left\langle X^{\prime} ; h, p_{h 1}\left|\hat{J}^{\mu}(0)\right| n, p_{n}\right\rangle \\
& \times\left\langle p, p_{p 1} ; n, p_{n} \mid d, p_{d}\right\rangle .
\end{aligned}
$$

FSIs between the slow hadron and the spectator are now incorporated by taking the hadron-spectator final state not as a product state (as is done in the IA) but as the scattering state generated by interactions between them. The boundary conditions for the scattering state correspond to the incomingwave solution [74],

$$
\left\langle p, p_{p} ; h, p_{h}\right| \rightarrow \text { in }\left\langle p, p_{p} ; h, p_{h}\right| .
$$

Following standard practice in nuclear physics we can express the wave function of this scattering state in terms of the effective interaction operator $\hat{T}$ (or $T$ matrix) corresponding to the interaction,

$$
\begin{aligned}
& \text { in }\left\langle p, p_{p} ; h, p_{h} \mid p, p_{p 1} ; h, p_{h 1}\right\rangle \\
& =\left\langle p, p_{p} ; h, p_{h} \mid p, p_{p 1} ; h, p_{h 1}\right\rangle \\
& -\frac{\left\langle p, p_{p} ; h, p_{h}|\hat{T}| p, p_{p 1} ; h, p_{h 1}\right\rangle}{\frac{1}{2}\left(p_{p}^{-}+p_{h}^{-}-p_{p 1}^{-}-p_{h 1}^{-}+i 0\right)} .
\end{aligned}
$$




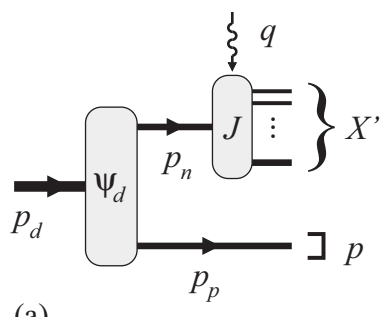

(a)

Equation (6.8) represents the LF analog of the LippmannSchwinger equation in nonrelativistic quantum mechanics. The denominator is the difference of LF energies between the initial and final states (the LF Hamiltonian in our convention is $\hat{H}_{\mathrm{LF}}=\frac{1}{2} \hat{P}_{\text {tot }}^{-}$; see Appendix 1 of Ref. [75]). In the context of a time-dependent formulation Eq. (6.8) can be regarded as the matrix element of the LF time evolution operator corresponding to the hadron-spectator interaction between LF time $x^{+}=0$ (when the current creates the state $h$ ) and $x^{+} \rightarrow+\infty$ (when the interactions are switched off),

$$
\begin{aligned}
\text { in } & \left\langle p, p_{p} ; h, p_{h} \mid p, p_{p 1} ; h, p_{h 1}\right\rangle \\
\quad & =\left\langle p, p_{p} ; h, p_{h}|\hat{U}(\infty, 0)| p, p_{p 1} ; h, p_{h 1}\right\rangle .
\end{aligned}
$$

This representation makes it obvious that the scattering state obeys incoming-wave boundary conditions. ${ }^{5}$

The effective interaction operator in Eq. (6.8) conserves the total LF momentum of the states. Using translational invariance, we can write the matrix element in the numerator of Eq. (6.8) in the form

$$
\left\langle p, p_{p} ; h, p_{h}|\hat{T}| p, p_{p 1} ; h, p_{h 1}\right\rangle=(2 \pi)^{3} \delta\left(p_{p}^{+}+p_{h}^{+}-p_{p 1}^{+}-p_{h 1}^{+}\right) \delta^{(2)}\left(\boldsymbol{p}_{p T}+\boldsymbol{p}_{h T}-\boldsymbol{p}_{p 1 T}-\boldsymbol{p}_{h 1 T}\right) T\left(p_{p}, p_{h} ; p_{p 1}, p_{h 1}\right),
$$

where no assumption is made about the LF energies of the initial and final states. The on-shell part of the scattering term in Eq. (6.8), in which the total final LF energy is equal to the initial one, is obtained by retaining the pole term of the energy denominator,

$$
\begin{aligned}
\frac{\left\langle p, p_{p} ; h, p_{h}|\hat{T}| p, p_{p 1} ; h, p_{h 1}\right\rangle}{\left.\frac{1}{2}\left(p_{p}^{-}+p_{h}^{-}-p_{p 1}^{-}-p_{h 1}^{-}-i 0\right)\right|_{\text {on-shell }}=} & i(2 \pi) \delta\left(p_{p}^{-}+p_{h}^{-}-p_{p 1}^{-}-p_{h 1}^{-}\right)\left\langle p, p_{p} ; h, p_{h}|\hat{T}| p, p_{p 1} ; h, p_{h 1}\right\rangle \\
= & i(2 \pi)^{4} \delta\left(p_{p}^{-}+p_{h}^{-}-p_{p 1}^{-}-p_{h 1}^{-}\right) \delta\left(p_{p}^{+}+p_{h}^{+}-p_{p 1}^{+}-p_{h 1}^{+}\right) \\
& \times \delta^{(2)}\left(\boldsymbol{p}_{p T}+\boldsymbol{p}_{h T}-\boldsymbol{p}_{p 1 T}-\boldsymbol{p}_{h 1 T}\right) T\left(p_{p}, p_{h} ; p_{p 1}, p_{h 1}\right) \\
= & i(2 \pi)^{4} \delta^{(4)}\left(p_{p}+p_{h}-p_{p 1}-p_{h 1}\right) \frac{1}{2} T\left(p_{p}, p_{h} ; p_{p 1}, p_{h 1}\right) .
\end{aligned}
$$

Here $T$ is the on-shell matrix element of the effective interaction, which describes the amplitude of the physical $p h \rightarrow p h$ scattering process and can be determined from experimental data; it is normalized such that, up to spin structures, it coincides with the invariant amplitude (see Appendix C). Equations (6.8)-(6.13) allow us to express the FSI matrix element in terms of the physical $p h \rightarrow p h$ amplitude within our scheme of approximations. The factor $1 / 2$ in Eq. (6.13) accounts for the fact that the interaction in the matrix element is present only from $x^{+}=0$ to $\infty$ (i.e., "half the time"), while in the scattering process it is present from $x^{+}=-\infty$ to $\infty$. We note that the same factor $1 / 2$ is obtained in an equivalent calculation of the FSI effect in the current matrix element using invariant perturbation theory, where it appears from the Cutkosky rules for the on-shell part of the Feynman diagram.

We can now derive from Eq. (6.6) the explicit expressions for the transition current to $X_{1}$ states. The $d \rightarrow p n$ matrix element in Eq. (6.6) is expressed in terms of the deuteron LF wave function Eq. (3.9). The FSI matrix element is substituted by Eq. (6.8). The noninteraction term on the right-hand side results in an expression of the same form as the IA result, Eq. (4.3). The interaction term is expressed in terms of the on-shell scattering amplitude using Eqs. (6.13). Altogether the transition current to $X_{1}$ states, Eq. (6.6), can be written as the sum of an IA and an FSI term,

$$
\begin{aligned}
\left\langle p X_{1}\left|\hat{J}^{\mu}(0)\right| d\right\rangle & \equiv\left\langle p h X^{\prime}\left|\hat{J}^{\mu}(0)\right| d\right\rangle=\langle\ldots\rangle[\mathrm{IA}]+\langle\ldots\rangle[\mathrm{FSI}], \\
\left\langle p h X^{\prime}\left|\hat{J}^{\mu}(0)\right| d\right\rangle[\mathrm{IA}] & =\frac{p_{d}^{+}}{p_{n}^{+}}\left\langle h, p_{h} ; X^{\prime}\left|\hat{J}^{\mu}(0)\right| n, p_{n}\right\rangle(2 \pi)^{3 / 2} \Psi_{d}\left(\alpha_{p}, \boldsymbol{p}_{p T}\right) \quad\left[p_{n}^{+}=p_{d}^{+}-p_{p}^{+}, \boldsymbol{p}_{n T}=-\boldsymbol{p}_{p T}\right],
\end{aligned}
$$

\footnotetext{
${ }^{5}$ The formal operator generating a two-body scattering state from the product states [cf. Eq. (6.8)] is known as the Møller operator and can be defined in a general context. Its representation as the limit of a time evolution operator depends on asymptotic conditions; for a discussion see Refs. [76-78].
} 


$$
\begin{aligned}
\left\langle p h X^{\prime}\left|\hat{J}^{\mu}(0)\right| d\right\rangle[\mathrm{FSI}]= & \int\left[d p_{p 1}\right] \frac{p_{d}^{+}}{p_{n}^{+}}\left\langle h, p_{h 1} ; X^{\prime}\left|\hat{J}^{\mu}(0)\right| n, p_{n}\right\rangle(2 \pi)^{3 / 2} \Psi_{d}\left(\alpha_{p 1}, \boldsymbol{p}_{p 1 T}\right) \\
& \times \frac{2 \pi}{p_{h 1}^{+}} \delta\left(p_{p}^{-}+p_{h}^{-}-p_{p 1}^{-}-p_{h 1}^{-}\right) \frac{i}{2} T\left(p_{p}, p_{h} ; p_{p 1}, p_{h 1}\right) \\
& {\left[p_{n}^{+}=p_{d}^{+}-p_{p 1}^{+}, \boldsymbol{p}_{n T}=-\boldsymbol{p}_{p 1 T} ; p_{h 1}^{+}=p_{h}^{+}+p_{p}^{+}-p_{p 1}^{+}, \boldsymbol{p}_{h 1 T}=\boldsymbol{p}_{h T}+\boldsymbol{p}_{p T}-\boldsymbol{p}_{p 1 T}\right] . }
\end{aligned}
$$

\section{B. Distorted spectral function}

The deuteron tensor for tagged DIS in the presence of FSIs is obtained as the product of the current matrix element and its complex conjugate, summed over all final states $X$, Eq. (2.19). In accordance with the distinction between final states with zero and one slow hadron, $X_{0}$ and $X_{1}$, we now write this sum as

$$
\begin{aligned}
W_{d}^{\mu \nu} & =\sum_{X_{0}}\left\langle d\left|\hat{J}^{\mu}(0)\right| p X_{0}\right\rangle\left\langle p X_{0}\left|\hat{J}^{\nu}(0)\right| d\right\rangle \\
& +\sum_{X_{1}}\left\langle d\left|\hat{J}^{\mu}(0)\right| p X_{1}\right\rangle\left\langle p X_{1}\left|\hat{J}^{\nu}(0)\right| d\right\rangle,
\end{aligned}
$$

and substitute the expressions Eqs. (6.5) and (6.14)-(6.16) for the different current matrix elements. It is easy to see that in Eq. (6.17) the sum over states $X_{0}$ (for which the current is always of IA form), and the part of the sum over states $X_{1}$ involving the IA term of the currents (in which no FSI takes place), reproduce the original IA result for the deuteron tensor, Eq. (4.4),

$$
\begin{aligned}
& \sum_{X_{0}}\left\langle d\left|\hat{J}^{\mu}(0)\right| p X_{0}\right\rangle[\mathrm{IA}]\left\langle p X_{0}\left|\hat{J}^{\nu}(0)\right| d\right\rangle[\mathrm{IA}] \\
+ & \sum_{X_{1}}\left\langle d\left|\hat{J}^{\mu}(0)\right| p X_{1}\right\rangle[\mathrm{IA}]\left\langle p X_{1}\left|\hat{J}^{\nu}(0)\right| d\right\rangle[\mathrm{IA}] \\
= & \sum_{X}\left\langle d\left|\hat{J}^{\mu}(0)\right| p X\right\rangle[\mathrm{IA}]\left\langle p X\left|\hat{J}^{\nu}(0)\right| d\right\rangle[\mathrm{IA}]=W_{d}^{\mu \nu}[\mathrm{IA}] .
\end{aligned}
$$

Here we have used that the sum over states $X_{0}$ and $X_{1}$ exhausts the full set of inclusive final states $X, \mathrm{Eq}$. (6.3).

Corrections to the IA tensor arise from the FSI terms in the currents in the sum over states $X_{1}$ in Eq. (6.17). These corrections come in two types: (a) the products of the FSI term of one current and the IA term of the other (linear FSIs); (b) the product of the FSI terms from both currents (quadratic FSIs).

Consider the linear FSI correction arising from the product of the FSI current, Eq. (6.16), and the complex conjugate IA current, Eq. (6.15). Because of the momentum integral in the FSI current, the momentum of the active neutron in the FSI current, $p_{n}^{+}$and $\boldsymbol{p}_{n T}$, is generally different from that in the IA current. The corresponding neutron current matrix elements can therefore not generally be combined to form the neutron tensor (which is diagonal in the neutron momentum), as was done for the IA in Eq. (4.4). An important simplification arises from the fact that the characteristic momenta in the deuteron wave function are much smaller than in the nucleon current matrix element. The latter is therefore not affected by the small shift of the active neutron momentum caused by the FSI integral and can be evaluated at the nominal active neutron momentum defined by the IA.
A similar simplification can be made regarding the slow hadron momentum in the neutron current matrix element. Under the FSI integral the slow hadron produced by the nucleon current has momentum $p_{h 1}^{+}$and $\boldsymbol{p}_{h 1 T}$, which differs from the momentum it has in the IA, $p_{h}^{+}$and $\boldsymbol{p}_{h T}$, by the momentum transfer through the rescattering process. Assuming that this momentum transfer is much smaller than the typical slow hadron momentum, we can evaluate the nucleon current at the nominal slow hadron momentum defined by the IA. Together, the two approximations imply that the nucleon current matrix elements are evaluated at the same nucleon and slow hadron momenta in both the FSI and IA, so that their product can be replaced by the nucleon tensor.

With these simplifications we can write the FSI term of the current matrix element, Eq. (6.16), in the form

$$
\begin{aligned}
\left\langle p h X^{\prime}\left|\hat{J}^{\mu}(0)\right| d\right\rangle[\mathrm{FSI}] & p_{d}^{+}\left\langle h, p_{h} ; X^{\prime}\left|\hat{J}^{\mu}(0)\right| n, p_{n}\right\rangle(2 \pi)^{3 / 2} i I_{d}, \\
= & \frac{p_{n}^{+}}{} \\
& {\left[p_{n}^{+}=p_{d}^{+}-p_{p}^{+}, \boldsymbol{p}_{n T}=-\boldsymbol{p}_{p T}\right], } \\
I_{d} \equiv & I_{d}\left(\alpha_{p}, \boldsymbol{p}_{p T}, \alpha_{h}, \boldsymbol{p}_{h T}\right) \\
\equiv & \int\left[d p_{p 1}\right] \frac{2 \pi}{p_{h 1}^{+}} \delta\left(p_{p}^{-}+p_{h}^{-}-p_{p 1}^{-}-p_{h 1}^{-}\right) \\
& \times \frac{1}{2} \Psi_{d}\left(\alpha_{p 1}, \boldsymbol{p}_{p 1 T}\right) T\left(p_{p}, p_{h} ; p_{p 1}, p_{h 1}\right) \\
& {\left[p_{h 1}^{+}=p_{h}^{+}+p_{p}^{+}-p_{p 1}^{+}, \boldsymbol{p}_{h 1 T}=\boldsymbol{p}_{h T}+\boldsymbol{p}_{p T}-\boldsymbol{p}_{p 1 T}\right] . }
\end{aligned}
$$

The function $I_{d}$ represents the integral of the deuteron LF wave function and the rescattering amplitude over the phase space available for the rescattering process, defined by the LF momenta of the final-state particles. Note that we have extracted the factor of $i$ from the rescattering integral and exhibit it explicitly in Eq. (6.19). The complete deuteron tensor emerging from the IA and FSI current matrix elements, Eqs. (6.14) and (6.19), including the pure IA contribution Eq. (6.18), is then obtained as

$$
\begin{aligned}
W_{d}^{\mu \nu} & \equiv W_{d}^{\mu \nu}\left(p_{d}, q, p_{p}\right) \\
= & W_{d}^{\mu \nu}[\mathrm{IA}]+W_{d}^{\mu v}[\mathrm{FSI}]+W_{d}^{\mu v}\left[\mathrm{FSI}^{2}\right] \\
W_{d}^{\mu v}[\mathrm{IA}]= & (2 \pi)^{3}\left(\frac{p_{d}^{+}}{p_{n}^{+}}\right)^{2}\left|\Psi_{d}\right|^{2} W_{n}^{\mu \nu}\left(p_{n}, \widetilde{q}\right) \\
W_{d}^{\mu \nu}[\mathrm{FSI}]= & (2 \pi)^{3}\left(\frac{p_{d}^{+}}{p_{n}^{+}}\right)^{2} \sum_{h} \int_{\text {phas }} d \Gamma_{h}(-2) \operatorname{Im}\left[\Psi_{d} I_{d}\right] \\
& \times W_{n, h}^{\mu \nu}\left(p_{n}, \widetilde{q}, p_{h}\right),
\end{aligned}
$$




$$
\begin{gathered}
W_{d}^{\mu v}\left[\mathrm{FSI}^{2}\right]= \\
(2 \pi)^{3}\left(\frac{p_{d}^{+}}{p_{n}^{+}}\right)^{2} \sum_{h} \int_{\text {phas }} d \Gamma_{h}\left|I_{d}\right|^{2} W_{n, h}^{\mu v}\left(p_{n}, \widetilde{q}, p_{h}\right) \\
{\left[p_{n}^{+}=p_{d}^{+}-p_{p}^{+}, \boldsymbol{p}_{n T}=0\right] .}
\end{gathered}
$$

The linear and quadratic FSI terms include integration over the phase space of the unobserved slow hadron $h$; the physical limits of the phase space are denoted by "phas" and will be specified below. In addition, they include the summation over the relevant slow hadron species $h$. From Eqs. (6.22)-(6.24) expressions for the tagged deuteron structure functions can be obtained by performing suitable projections (see Appendix B). The operations are the same as in the IA calculation in Sec. IVD. When applying the projections, the conditional neutron tensor produces the conditional neutron structure functions,

$$
\begin{aligned}
W_{n, h}^{\mu v}\left(p_{n}, \widetilde{q}, p_{h}\right) \rightarrow & F_{2 n, h}\left(\tilde{x}, Q^{2} ; \zeta_{h}, p_{h T}\right) \\
= & F_{2 n}\left(\tilde{x}, Q^{2}\right) D_{h}\left(\tilde{x}, Q^{2} ; \zeta_{h}, p_{h T}\right), \text { etc. } \\
& {\left[\widetilde{x}=x /\left(2-\alpha_{p}\right)\right] . }
\end{aligned}
$$

The tagged deuteron structure functions can be expressed in a form analogous to the IA, Eq. (4.20), in terms of a "distorted" spectral function,

$$
\begin{aligned}
F_{2 d}\left(x, Q^{2} ; \alpha_{p}, p_{p T}\right) & =S_{d}\left(\alpha_{p}, \boldsymbol{p}_{p T}\right)[\operatorname{dist}] F_{2 n}\left(\tilde{x}, Q^{2}\right) \\
S_{d}[\mathrm{dist}] & \equiv S_{d}[\mathrm{IA}]+S_{d}[\mathrm{FSI}]+S_{d}\left[\mathrm{FSI}^{2}\right] .
\end{aligned}
$$

The explicit expressions of the terms are

$$
\begin{aligned}
S_{d}[\mathrm{IA}] & =\frac{\left|\Psi_{d}\right|^{2}}{2-\alpha_{p}} \\
S_{d}[\mathrm{FSI}] & =\frac{1}{2-\alpha_{p}} \sum_{h} \int_{\text {phas }} d \Gamma_{h} D_{h}(-2) \operatorname{Im}\left[\Psi_{d} I_{d}\right] \\
S_{d}\left[\mathrm{FSI}^{2}\right] & =\frac{1}{2-\alpha_{p}} \sum_{h} \int_{\text {phas }} d \Gamma_{h} D_{h}\left|I_{d}\right|^{2}
\end{aligned}
$$

It remains to determine the kinematic limits of the phase space integral over the final-state slow hadron LF momentum in Eqs. (6.29) and (6.30), or Eqs. (6.23) and (6.24). We work in a collinear frame (cf. Sec. IIC) and parametrize the LF plus momentum of the slow hadron and the recoil nucleon as fractions of $p_{d}^{+} / 2$,

$$
p_{h}^{+}=\frac{\alpha_{h} p_{d}^{+}}{2}, \quad p_{p}^{+}=\frac{\alpha_{p} p_{d}^{+}}{2} .
$$

On general grounds the plus momentum fractions of the slow hadron and the recoil nucleon in the final state of DIS on the deuteron are bounded by [cf. Sec. V A and Eq. (5.3); we approximate $\xi_{d} \approx x_{d}$ and set $x_{d}=x / 2$, cf. Eq. (2.7)]

$$
0<\alpha_{h}+\alpha_{p}<2\left(1-x_{d}\right)=2-x .
$$

For given $\alpha_{p}$, the phase space integral over the slow hadron momentum is therefore restricted to

$$
\alpha_{h}<2-\alpha_{p}-x .
$$

The LF momentum fraction of the slow hadron with respect to the active nucleon is

$$
\zeta_{h}=\frac{p_{h}^{+}}{p_{d}^{+}-p_{p}^{+}}=\frac{\alpha_{h}}{2-\alpha_{p}} .
$$

From Eq. (6.33) it follows that

$$
\zeta_{h}=\frac{\alpha_{h}}{2-\alpha_{p}}<1-\frac{x}{2-\alpha_{p}}=1-\tilde{x},
$$

as it should be for the DIS final state on the nucleon, cf. Eq. (5.3). Thus $\zeta_{h}$ has the correct kinematic limits within our scheme of approximations.

Equation (6.34) describes the effect of the longitudinal motion of the active neutron on the slow hadron distribution emerging from the DIS process. Note that we neglect the effect of the transverse motion of the active neutron on the slow hadron distribution, consistently with our treatment of the transverse momentum dependence of the neutron tensor in the IA (see Sec. IVC) and our definition of the distorted spectral function (see Sec. VIB). These effects are small because the average neutron momentum in the deuteron is much smaller than the typical transverse momentum of the DIS hadrons, $\left|\boldsymbol{p}_{n T}\right|^{2} \ll 1 / B_{h}$ (see Sec. VC).

\section{Spin degrees of freedom}

We now include the internal spin degrees of freedom in the FSI current matrix element and the deuteron tensor, following the same approach as in the IA in Sec. IV E. The expressions for the deuteron tensor Eqs. (6.22)-(6.24) take the form (we show only the spin quantum numbers and suppress all other arguments)

$$
\begin{aligned}
W_{d}^{\mu \nu}[\mathrm{IA}] & =[\ldots] \frac{1}{3} \sum_{\lambda_{d}} \sum_{\sigma_{p}} \sum_{\sigma_{n 1} \sigma_{n 2}} \Psi_{d}^{*}\left(\sigma_{p}, \sigma_{n 2} \mid \lambda_{d}\right) \Psi_{d}\left(\sigma_{p}, \sigma_{n 1} \mid \lambda_{d}\right) W_{n}^{\mu \nu}\left(\sigma_{n 2}, \sigma_{n 1}\right) \\
W_{d}^{\mu \nu}[\mathrm{FSI}] & =[\ldots] \frac{1}{3} \sum_{\lambda_{d}} \sum_{\sigma_{p}} \sum_{\sigma_{n 1} \sigma_{n 2}} \sum_{h} \int_{\text {phas }} d \Gamma_{h}\left[i \Psi_{d}^{*}\left(\sigma_{p}, \sigma_{n 2} \mid \lambda_{d}\right) I_{d}\left(\sigma_{p}, \sigma_{n 1} \mid \lambda_{d}\right)-i I_{d}^{*}\left(\sigma_{p}, \sigma_{n 2} \mid \lambda_{d}\right) \Psi_{d}\left(\sigma_{p}, \sigma_{n 1} \mid \lambda_{d}\right)\right] W_{n, h}^{\mu \nu}\left(\sigma_{n 2}, \sigma_{n 1}\right), \\
W_{d}^{\mu v}\left[\mathrm{FSI}^{2}\right] & =[\ldots] \frac{1}{3} \sum_{\lambda_{d}} \sum_{\sigma_{p}} \sum_{\sigma_{n 1} \sigma_{n 2}} \sum_{h} \int_{\text {phas }} d \Gamma_{h} I_{d}^{*}\left(\sigma_{p}, \sigma_{n 2} \mid \lambda_{d}\right) I_{d}\left(\sigma_{p}, \sigma_{n 1} \mid \lambda_{d}\right) W_{n, h}^{\mu \nu}\left(\sigma_{n 2}, \sigma_{n 1}\right) \\
{[\ldots] } & =(2 \pi)^{3}\left(p_{d}^{+} / p_{n}^{+}\right)^{2} .
\end{aligned}
$$


The deuteron tensor is averaged over the initial deuteron helicity $\lambda_{d}$ and summed over the final proton spin $\sigma_{p}$, and involves the summation over the internal neutron spins $\sigma_{n 1}$ and $\sigma_{n 2}$. Here

$$
\Psi_{d}\left(\sigma_{p}, \sigma_{n 1} \mid \lambda_{d}\right) \equiv \Psi_{d}\left(\alpha_{p}, \boldsymbol{p}_{p T} ; \sigma_{p}, \sigma_{n 1} \mid \lambda_{d}\right)
$$

is the spin-dependent deuteron LF wave function Eq. (3.33), and

$$
\begin{aligned}
I_{d}\left(\sigma_{p}, \sigma_{n 1} \mid \lambda_{d}\right) & \equiv I_{d}\left(\alpha_{p}, \boldsymbol{p}_{p T}, \alpha_{h}, \boldsymbol{p}_{h T} ; \sigma_{p}, \sigma_{n 1} \mid \lambda_{d}\right) \\
& \equiv \sum_{\sigma_{p 1}} \int\left[d p_{p 1}\right] \frac{2 \pi}{p_{h 1}^{+}} \delta\left(p_{p}^{-}+p_{h}^{-}-p_{p 1}^{-}-p_{h 1}^{-}\right) \frac{1}{2} \Psi_{d}\left(\alpha_{p 1}, \boldsymbol{p}_{p 1 T} ; \sigma_{p 1}, \sigma_{n 1} \mid \lambda_{d}\right) T\left(p_{p}, p_{h} ; p_{p 1}, p_{h 1} ; \sigma_{p 1}, \sigma_{p}\right)
\end{aligned}
$$

is the spin-dependent rescattering integral, which involves summation over the internal proton spin $\sigma_{p 1}$. These expressions represent the general result for FSIs in tagged DIS on the deuteron within our picture.

In Eqs. (6.36)-(6.38) the LF helicities of the neutrons, $\sigma_{n 1}$ and $\sigma_{n 2}$, are summed over independently in the current matrix element and its complex conjugate, and the neutron tensors generally depend on both spin variables. In the IA cross section Eq. (6.36) the neutron tensor is the one for inclusive scattering (no identified hadron in the final state), $W_{n}^{\mu \nu}$. This tensor is diagonal in the neutron LF helicity, since off-diagonal terms would amount to a transverse spin dependence of the inclusive cross section, which is forbidden by symmetries [see Eq. (4.36) and Sec. IVE],

$$
W_{n}^{\mu \nu}\left(p_{n}, \tilde{q} ; \sigma_{n 2}, \sigma_{n 1}\right) \propto \delta\left(\sigma_{n 2}, \sigma_{n 1}\right) .
$$

In the FSI cross sections Eqs. (6.37) and (6.38) the neutron tensor is the one for conditional scattering (identified hadron $h$ in the final state), $W_{n, h}^{\mu \nu}$. This tensor could in principle have off-diagonal elements through a correlation of the neutron transverse spin with the hadron transverse momentum. In the following we neglect such correlations and assume that the conditional neutron scattering tensor is diagonal in the neutron LF helicity in the same way as the inclusive one,

$$
W_{n, h}^{\mu \nu}\left(p_{n}, \tilde{q}, p_{h} ; \sigma_{n 2}, \sigma_{n 1}\right) \propto \delta\left(\sigma_{n 2}, \sigma_{n 1}\right) .
$$

This approximation is consistent with our neglecting other transverse-momentum dependent structures in the conditional neutron tensor. The effects of these structures under the rescattering integral are suppressed because they average out, particularly for small external recoil momenta $\boldsymbol{p}_{p}$. Moreover, we retain only the unpolarized part of the neutron tensor, which is independent of the diagonal spin quantum number $\sigma_{n 1}$ and involves the unpolarized neutron structure functions Eq. (4.14).

With these approximations we can again express the tagged deuteron structure functions with FSIs in terms of a distorted spectral function, Eq. (6.27), which is now obtained as

$$
\begin{aligned}
S_{d}[\mathrm{IA}]= & \frac{1}{2-\alpha_{p}} \frac{1}{3} \sum_{\lambda_{d}} \sum_{\sigma_{p} \sigma_{n}} \Psi_{d}^{*}\left(\sigma_{p}, \sigma_{n} \mid \lambda_{d}\right) \Psi_{d}\left(\sigma_{p}, \sigma_{n} \mid \lambda_{d}\right), \\
S_{d}[\mathrm{FSI}]= & \frac{1}{2-\alpha_{p}} \frac{1}{3} \sum_{\lambda_{d}} \sum_{\sigma_{p} \sigma_{n}} \sum_{h} \int_{\text {phas }} d \Gamma_{h} \\
& \times\left[i \Psi_{d}^{*}\left(\sigma_{p}, \sigma_{n} \mid \lambda_{d}\right) I_{d}\left(\sigma_{p}, \sigma_{n} \mid \lambda_{d}\right)\right. \\
& \left.-i I_{d}^{*}\left(\sigma_{p}, \sigma_{n} \mid \lambda_{d}\right) \Psi_{d}\left(\sigma_{p}, \sigma_{n} \mid \lambda_{d}\right)\right],
\end{aligned}
$$

$$
\begin{aligned}
S_{d}\left[\mathrm{FSI}^{2}\right]= & \frac{1}{2-\alpha_{p}} \frac{1}{3} \sum_{\lambda_{d}} \sum_{\sigma_{p} \sigma_{n}} \sum_{h} \int_{\text {phas }} d \Gamma_{h} \\
& \times I_{d}^{*}\left(\sigma_{p}, \sigma_{n} \mid \lambda_{d}\right) I_{d}\left(\sigma_{p}, \sigma_{n} \mid \lambda_{d}\right) .
\end{aligned}
$$

These expressions generalize the spinless result of Eqs. (6.28)(6.30).

\section{Nonrelativistic approximation}

We evaluate the FSI correction using the nonrelativistic approximation for the deuteron LF wave function and a corresponding approximation for the rescattering integral. This approximation will be justified a posteriori, by verifying that the dominant momenta in the rescattering integral are $\left|\boldsymbol{p}_{p 1}\right|^{2} \ll$ $M_{N}^{2}$.

To derive the nonrelativistic approximation we convert the rescattering integral Eq. (6.41) to a manifestly rotationally invariant form. Here we use the fact that the integral over the LF momentum of the intermediate proton, together with the LF-energy conserving delta function, is an invariant phase space integral and does not depend on the LF direction; only the integrand depends on LF direction. Using the condition of four-momentum conservation

$$
p_{p 1}+p_{h 1}=p_{p}+p_{h}
$$

one easily shows that

$$
\begin{aligned}
& \frac{1}{p_{h 1}^{+}} \delta\left(p_{h 1}^{-}+p_{p 1}^{-}-p_{h}^{-}-p_{p}^{-}\right) \\
& \quad=\delta\left[p_{h 1}^{+}\left(p_{h 1}^{-}+p_{p 1}^{-}-p_{h}^{-}-p_{p}^{-}\right)\right] \\
& \quad=\delta\left[\left(p_{h}+p_{p}-p_{p 1}\right)^{2}-M_{h}^{2}\right],
\end{aligned}
$$

i.e., the LF energy-conserving delta function can be expressed as the mass shell delta function for the four-vector sum of three of the momenta in the scattering process. The integral in Eq. (6.41) can therefore be written in the form

$$
\begin{aligned}
& \int\left[d p_{p 1}\right] \frac{2 \pi}{p_{h 1}^{+}} \delta\left(p_{h 1}^{-}+p_{p 1}^{-}-p_{h}^{-}-p_{p}^{-}\right)[\ldots] \\
& =\int \frac{d^{3} p_{p 1}}{(2 \pi)^{3} 2 E_{N}\left(\boldsymbol{p}_{1}\right)} 2 \pi \delta\left[\left(p_{h}+p_{p}-p_{p 1}\right)^{2}-M_{h}^{2}\right][\ldots] \\
& \equiv \int\left[d p_{p 1}\right]_{\text {shell }}[\ldots] .
\end{aligned}
$$

The integral Eq. (6.49) can now be evaluated in a nonrelativistic approximation. In the phase space element we replace $E_{N}\left(\boldsymbol{p}_{p 1}\right) \rightarrow M_{N}$. The mass-shell delta function in Eq. (6.49) 
is resolved assuming proton momenta $\left|\boldsymbol{p}_{p 1}\right|,\left|\boldsymbol{p}_{p}\right| \ll M_{N}$ (nonrelativistic dispersion), but placing no restriction on the hadron momenta $\left|\boldsymbol{p}_{h 1}\right|,\left|\boldsymbol{p}_{h}\right|$ (relativistic dispersion); the procedure is described in Appendix D.

In the integrand of Eq. (6.41) we replace the LF wave function by the nonrelativistic approximation Eq. (3.29). The rescattering integral becomes

$$
\begin{aligned}
& I_{d}\left(\boldsymbol{p}_{p}, \boldsymbol{p}_{h} ; \sigma_{p}, \sigma_{n}\right) \\
& =\sum_{\sigma_{p 1}} \int\left[d p_{p 1}\right]_{\text {shell }} \sqrt{M_{N}}\left(1-\frac{p_{p 1}^{z}}{M_{N}}\right) \frac{1}{2} \widetilde{\Psi}_{d}\left(\boldsymbol{p}_{p 1} ; \sigma_{p 1}, \sigma_{n}\right) \\
& \quad \times T\left(\boldsymbol{p}_{p}, \boldsymbol{p}_{h} ; \boldsymbol{p}_{p 1}, \boldsymbol{p}_{h 1} ; \sigma_{p 1}, \sigma_{p}\right) .
\end{aligned}
$$

We neglect the spin rotations resulting from the fact that proton and neutron momentum in FSI amplitude are not the same as in the IA. Such effects represent relativistic corrections to the integrand of the FSI integral, which become noticeable only at large integration momenta $\left|\boldsymbol{p}_{p 1}\right|$. Our studies below show that the rescattering integral is dominated by momenta $\left|\boldsymbol{p}_{p 1}\right|^{2} \ll M_{N}^{2}$, so that it is justified to neglect these corrections.

To proceed further we need to model the spin dependence of the rescattering amplitude. Here we assume that the hadronproton scattering amplitude preserves the proton spin

$$
\begin{aligned}
T\left(\boldsymbol{p}_{p}, \boldsymbol{p}_{h} ; \boldsymbol{p}_{p 1}, \boldsymbol{p}_{h 1} ; \sigma_{p 1}, \sigma_{p}\right) & \rightarrow T\left(s_{p h}, t_{1}\right) \delta\left(\sigma_{p 1}, \sigma_{p}\right), \\
t_{1} & \equiv-\left(\boldsymbol{p}_{p 1}-\boldsymbol{p}\right)^{2},
\end{aligned}
$$

where $T\left(s_{p h}, t_{1}\right)$ is the invariant amplitude described in Appendix C. Recall that we regard the slow hadron beam as spinless, so that spin-spin interactions are excluded. A dependence of the rescattering amplitude on the proton spin could in principle arise from spin-orbit interactions; such effects are suppressed at small proton momenta and of the same order as other spin-orbit effects which we neglect.

With these simplifications it is straightforward to perform the summation over internal spin variables and compute the distorted spectral function in the nonrelativistic approximation. The result is

$$
\begin{aligned}
S_{d}[\mathrm{dist}]= & S_{d}[\mathrm{IA}]+S_{d}[\mathrm{FSI}]+S_{d}\left[\mathrm{FSI}^{2}\right], \\
\frac{S_{d}[\mathrm{IA}]}{2-\alpha_{p}}= & M_{N} \rho_{d}\left(\boldsymbol{p}_{p}, \boldsymbol{p}_{p}\right), \\
\frac{S_{d}[\mathrm{FSI}]}{2-\alpha_{p}}= & \sum_{h} \int_{\text {phas }} d \Gamma_{h} D_{h} \int\left[d p_{p 1}\right]_{\text {shell }}\left(1+\frac{p_{p}^{z}-p_{p 1}^{z}}{M_{N}}\right) \\
& \times M_{N} \rho_{d}\left(\boldsymbol{p}_{p 1}, \boldsymbol{p}_{p}\right)\left[-\operatorname{Im} T\left(s_{p h}, t_{1}\right)\right], \\
\frac{S_{d}\left[\mathrm{FSI}^{2}\right]}{2-\alpha_{p}}= & \sum_{h} \int_{\text {phas }} d \Gamma_{h} D_{h} \int\left[d p_{p 2}\right]_{\text {shell }}\left(1+\frac{p_{p}^{z}-p_{p 2}^{z}}{M_{N}}\right) \\
& \times \int\left[d p_{p 1}\right]_{\text {shell }}\left(1+\frac{p_{p}^{z}-p_{p 1}^{z}}{M_{N}}\right) \\
& \times M_{N} \rho_{d}\left(\boldsymbol{p}_{p 2}, \boldsymbol{p}_{p 1}\right) \frac{1}{4} T^{*}\left(s_{p h}, t_{2}\right) T\left(s_{p h}, t_{1}\right) .(6.55)
\end{aligned}
$$

These results generalize the IA result in the nonrelativistic approximation, Eq. (4.43). We quote the expressions for spectral function divided by $2-\alpha_{p}$, which in the IA coincides with the nonrelativistic momentum density. The function $\rho_{d}\left(\boldsymbol{p}_{p 1}, \boldsymbol{p}_{p}\right)$ in Eq. (6.54) is the nondiagonal spin-averaged momentum density of the unpolarized deuteron

$$
\begin{aligned}
\rho_{d}\left(\boldsymbol{p}_{p 1}, \boldsymbol{p}_{p}\right)= & \frac{1}{3} \sum_{\lambda_{d}} \sum_{\sigma_{p}, \sigma_{n}} M_{N} \widetilde{\Psi}_{d}\left(\boldsymbol{p}_{p 1} ; \sigma_{p}, \sigma_{n} \mid \lambda_{d}\right) \\
& \times \widetilde{\Psi}_{d}^{*}\left(\boldsymbol{p}_{p} ; \sigma_{p}, \sigma_{n} \mid \lambda_{d}\right) \\
= & U\left(p_{p 1}\right) U\left(p_{p}\right)+\frac{1}{2}\left[\frac{3\left(\boldsymbol{p}_{p 1} \boldsymbol{p}_{p}\right)^{2}}{p_{p 1}^{2} p_{p}^{2}}-1\right] \\
& \times W\left(p_{p 1}\right) W\left(p_{p}\right) ;
\end{aligned}
$$

$\rho_{d}\left(\boldsymbol{p}_{p 1}, \boldsymbol{p}_{p 2}\right)$ in Eq. (6.55) is defined analogously. The factors depending on the $z$ components of the proton momenta arise from the nonrelativistic approximation to the LF wave function, cf. Eq. (3.37), and the flux factors, and represent the ratios

$$
\frac{2-\alpha_{p 1}}{2-\alpha_{p}}=1+\frac{p_{p}^{z}-p_{p 1}^{z}}{M_{N}}+O\left(\frac{|\boldsymbol{p}|^{2}}{M_{N}^{2}}\right), \text { etc. }
$$

The FSI corrections Eqs. (6.54) and (6.55) are diagonal in the $S$ and $D$ waves, i.e., they involve terms quadratic in the $S$ - and $D$-wave functions but no interference terms. This is a consequence of the spin structure of the nonrelativistic deuteron, where $S$ and $D$ waves do not mix in the momentum density (see Appendix A), and of our assumption regarding the spin dependence of the rescattering process, Eq. (6.51). The dominant FSI effect at small proton momenta $\left|\boldsymbol{p}_{p}\right| \leqslant$ $200 \mathrm{MeV}$ therefore comes from the $S$-wave IA interfering with the $S$-wave FSI amplitude in Eqs. (6.54); this contribution could be calculated also without recourse to the nonrelativistic approximation, using the expressions of Sec. VIB. At larger proton momenta the quadratic FSI correction Eqs. (6.55) becomes comparable to the linear one, with the dominant contribution coming from the product of the $S$-wave integrals. Numerical results are presented in Sec. VIF below.

We now want to quantify what values of intermediate proton momenta $\left|\boldsymbol{p}_{p 1}\right|$ effectively contribute to the rescattering integrals, and how the integrals converge at large $\left|\boldsymbol{p}_{p 1}\right|$. The integration over the two-dimensional phase space defined by Eq. (6.49) results in radial integrals of the type

$$
\int d p_{p 1} p_{p 1} U\left(p_{p 1}\right), \quad \int d p_{p 1} p_{p 1} W\left(p_{p 1}\right) ;
$$

the actual integration limits resulting from energy conservation are given in Appendix D. The convergence of the integrals at large $p_{p 1}$ is ensured by the rapid fall-off of the radial wave functions. Additional suppression at large $p_{p 1}$ results from the $t_{1}$ dependence of the rescattering amplitude,

$$
t_{1}=-\left(\boldsymbol{p}_{p 1}-\boldsymbol{p}_{p}\right)^{2}, \quad T\left(s_{p h}, t_{1}\right) \propto e^{-b t_{1}} .
$$

Figure 12 shows the radial integrands in Eq. (6.59) as functions of $p_{p 1}$, both with and without the exponential factor resulting from the rescattering amplitude (here the external proton momentum is chosen as $\boldsymbol{p}_{p}=0$, such that $t_{1}=-p_{p}^{2}$ ). One sees that the integrands decrease rapidly at large $p_{p 1}$ for both $S$ and $D$ waves, and that the effective contributions arise from momenta $p_{p 1} \ll 500 \mathrm{MeV}$, where the nonrelativistic 


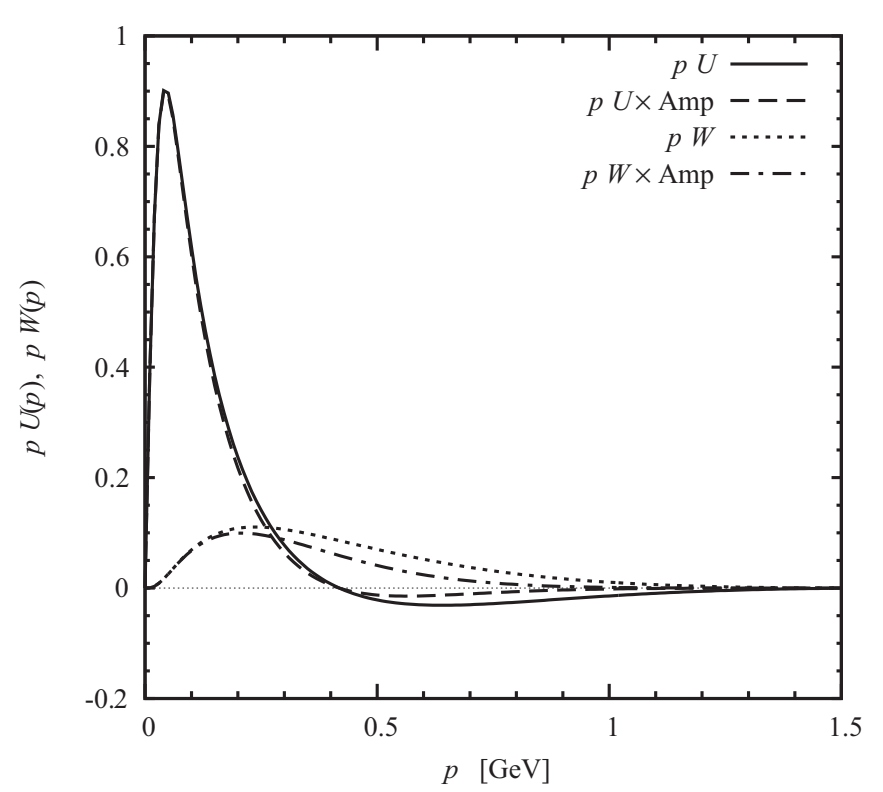

FIG. 12. Momentum distributions in the radial integrals in the rescattering integral, Eq. (6.59). Shown are the integrands as functions of the intermediate proton momentum $p \equiv\left|\boldsymbol{p}_{p 1}\right|$, evaluated with the AV18 wave functions. Red and blue lines: $S$ and $D$ waves. Solid and dashed lines: Momentum dependence without and with the $t_{1}$ dependence of the rescattering amplitude.

approximation is well justified. Similar behavior is obtained for nonzero external proton momenta $\left|\boldsymbol{p}_{p}\right| \lesssim 200 \mathrm{MeV}$, which are the values we consider in the present study.

\section{E. Positivity properties}

Some comments are in order regarding the sign of the FSI correction and the positivity of the spectral function. The imaginary part of the elastic rescattering amplitude is related to the total proton-hadron cross section at the given energy by the optical theorem (cf. Appendix $\mathrm{C}$ for the case that the hadron is a nucleon, $h=p, n)$, and therefore satisfies

$$
\operatorname{Im} T\left(s_{p h}, t_{1}=0\right)>0 .
$$

As a result, the contribution to the spectral function that arises from the interference of the $S$-wave FSI and the $S$-wave IA amplitudes, Eq. (6.54), is explicitly negative,

$$
S_{d}[\mathrm{FSI}]<0
$$

this holds at proton momenta $\left|\boldsymbol{p}_{p}\right|<300 \mathrm{MeV}$, where the $S$-wave radial function is positive. In contrast, the contribution that arises from the square of the $S$-wave FSI amplitudes, Eq. (6.55), is explicitly positive,

$$
S_{d}\left[\mathrm{FSI}^{2}\right]>0 .
$$

These findings have a simple interpretation in terms of conventional quantum-mechanical scattering theory. The linear term in the FSI amplitude represents the loss of flux due to absorption of the outgoing hadron-nucleon wave at a given value of the final nucleon momentum. The quadratic term represents the gain in cross section due to scattering of the outgoing wave into a configuration with the given value of the final nucleon momentum. In the language of wave optics, the two effects can be referred to as "absorption" and "refraction." One expects that absorption is the dominant effect at low recoil momenta, while refraction becomes dominant at large recoil momenta. This expectation is borne out by the numerical results described below.

The total distorted spectral function must be positive,

$$
S_{d}[\text { dist }]>0,
$$

as it represents the physical cross section for tagged DIS on the deuteron. In our scheme this is ensured by the fact that the hadronic tensor (i.e., the cross section) is calculated as the square of the current matrix element with the outgoing distorted wave. The further approximations made in the distorted spectral function do not change this basic property. Because the linear term in the FSI amplitude is negative, Eq. (6.62), both the linear and quadratic terms are needed to ensure positivity of the overall spectral function. This is again demonstrated by the numerical results.

\section{F. Recoil momentum dependence}

We now evaluate the distorted spectral function numerically, using the nonrelativistic approximation of Sec. VID, and study the magnitude of the distortion and its kinematic dependence on the recoil proton momentum. The parameters of the slow-hadron distribution and the rescattering amplitude are described in Sec. VD and Appendix C. Throughout we consider a value of $x \sim \xi \sim 0.1$, which is so small that it does not significantly restrict the slow hadron momentum, so that the integration can be carried out over the full range $0<\zeta_{h}<1$ [cf. Eq. (5.3) and Figs. 9 and 10]; the calculations can easily be extended to larger values of $x$.

The spectral function can be studied as a function of any of the recoil momentum variables described in Sec. IID. The most transparent representation is obtained using as independent variables the modulus of the recoil momentum in the deuteron rest frame and its angle relative to the $\boldsymbol{q}$ vector, Eq. (2.61),

$$
p_{p} \equiv\left|\boldsymbol{p}_{p}(\mathrm{RF})\right|, \quad \cos \theta_{p q}=-\frac{p_{p}^{z}(\mathrm{RF})}{\left|\boldsymbol{p}_{p}(\mathrm{RF})\right|} .
$$

Their relation to the variables $t^{\prime}$ and $\alpha_{p}$ is given by Eqs. (2.53) and (2.62).

Figure 13 shows the ratio of the linear FSI term in the distorted spectral function, $S_{d}$ [FSI], Eq. (6.54), to the IA term, $S_{d}$ [IA], Eq. (6.53), as a function of $\cos \theta_{p q}$, for several values of $p_{p}$. This ratio describes the relative correction to the IA arising from the linear FSI term. The plot shows the $S_{d}[\mathrm{FSI}]$ obtained from the $S$-wave only and the total from $S+D$ waves; the $S_{d}[\mathrm{IA}]$ in the denominator is always the total from $S+D$ waves. The following features are apparent:

(i) The correction from the linear FSI term is negative and increases in magnitude with the recoil momentum, from about $-5 \%$ at $p_{p} \sim 100 \mathrm{MeV}$ to about $-20 \%$ at $\sim 200 \mathrm{MeV}$.

(ii) At small $p_{p}$ the correction is isotropic. At $p_{p}>$ $200 \mathrm{MeV}$ it develops a peak at sideways angles, $\cos \theta_{p q} \sim 0$, and a forward-backward asymmetry.

(iii) The $S$ wave completely dominates the linear FSI term up to $p_{p} \sim 200 \mathrm{MeV}$; the $D$ wave becomes noticeable only at larger momenta. 


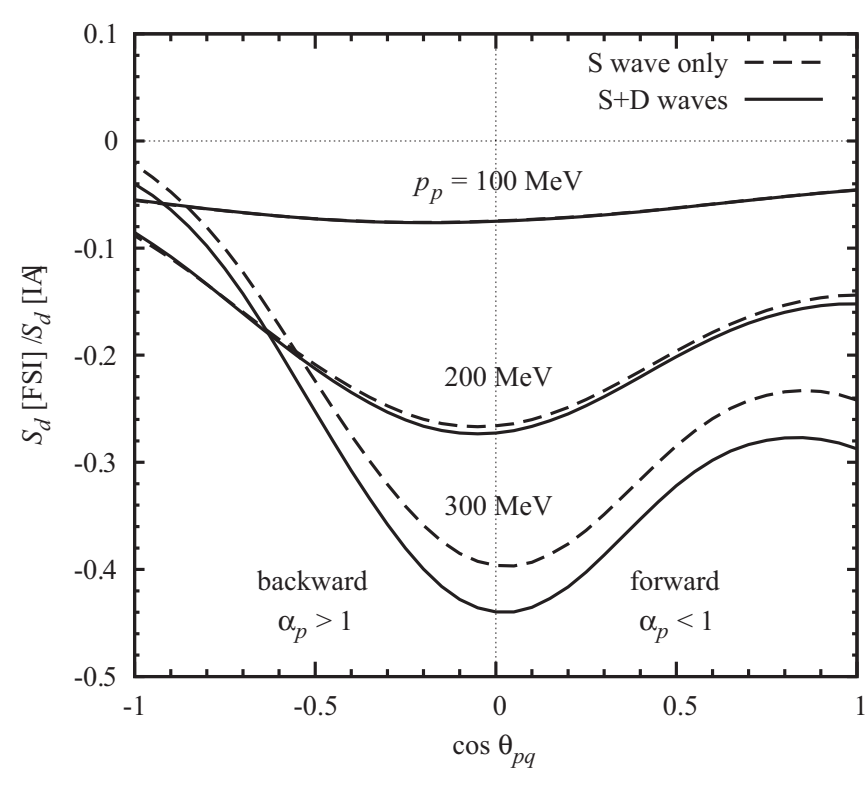

FIG. 13. The ratio of the linear FSI and IA deuteron spectral functions, $S_{d}[\mathrm{FSI}] / S_{d}[\mathrm{IA}]$, Eqs. (6.53) and (6.54), as a function of the cosine of the recoil momentum angle in the deuteron rest frame, $\cos \theta_{p q}$, for several values of the recoil momentum modulus $p_{p} \equiv\left|\boldsymbol{p}_{p}\right|$, as indicated on the plot. The plot shows the $S_{d}[\mathrm{FSI}]$ obtained from the $S$ wave only and the total from $S+D$ waves; the $S_{d}[\mathrm{IA}]$ in the denominator is always the total from $S+D$ waves. Note that the ratio shown here includes only the term linear in the FSI amplitude, not the quadratic one.

Figure 14 shows the ratio of the entire distorted spectral function, $S_{d}$ [dist], Eqs. (6.52), including the IA, FSI, and FSI ${ }^{2}$ terms, Eqs. (6.53)-(6.55), to the IA spectral function $S_{d}[\mathrm{IA}]$. In other words, it shows the factor by which the IA spectral function is modified by the entire FSI effect. The following features are apparent:

(i) At low recoil momenta $p_{p} \lesssim 300 \mathrm{MeV}$ the linear FSI term dominates and the FSI effect is mainly absorptive, reducing the spectral function relative to the IA. In this region the distorted spectral function has a minimum at $\cos \theta_{p q} \sim 0$.

(ii) At higher recoil momenta $p_{p} \gtrsim 300 \mathrm{MeV}$ the $\mathrm{FSI}^{2}$ term takes over at forward and sideways angles, $\cos \theta_{p q}>-0.7$, resulting in a large positive correction relative to the IA. The distorted spectral function now shows a maximum at slightly forward angles $\cos \theta_{p q} \sim 0.2$. The transition between the lowand high-momentum regimes is rather sudden.

(iii) At backward angles $\cos \theta_{p q}<-0.7$ the $\mathrm{FSI}^{2}$ term is suppressed, so that the FSI remains absorptive even at large recoil momenta. The spectral function in this region shows little variation with the recoil momentum at $p_{p} \gtrsim 300 \mathrm{MeV}$. Overall this results in a forward-backward asymmetry of the spectral function at large momenta.

(iv) The distorted spectral function is positive for all recoil momenta $\boldsymbol{p}_{p}$, as required on general grounds, cf. Eq. (6.64).

The observed dependencies are naturally explained by considering the kinematics of the scattering process in the deuteron rest frame. At low recoil momenta the main rescattering effect is always at $\theta_{p q} \sim 90^{\circ}$, because the only way in which the forward-moving DIS hadron with momentum $\sim 1 \mathrm{GeV}$ could

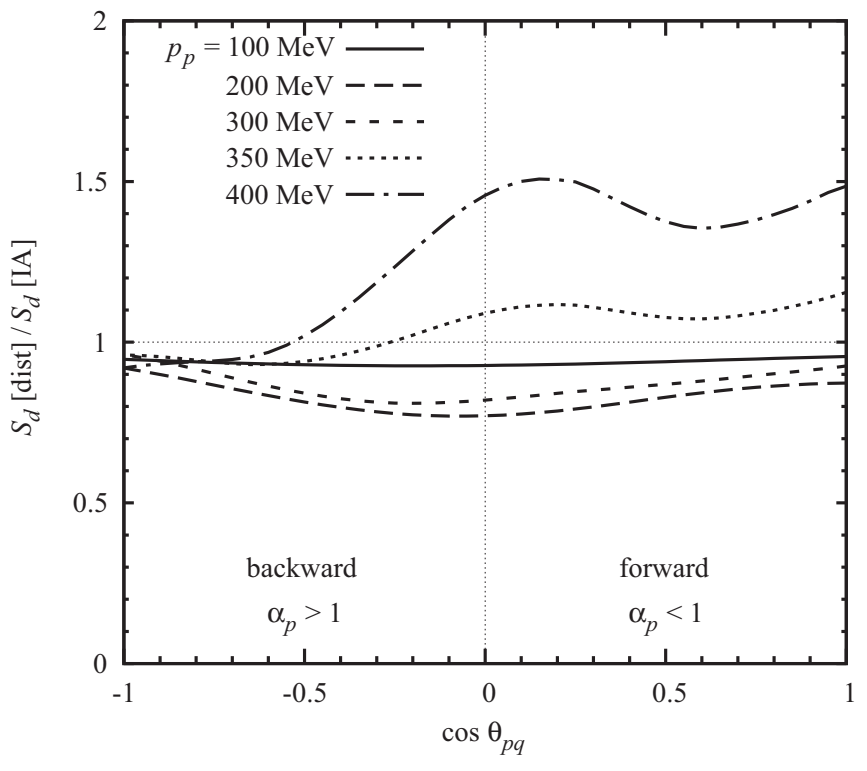

FIG. 14. The ratio of the distorted spectral function to the IA spectral function, $S_{d}$ [dist] $/ S_{d}$ [IA], Eqs. (6.52)-(6.55), as a function of the cosine of the recoil momentum angle in the deuteron rest frame, $\cos \theta_{p q}$, for several values of the recoil momentum modulus $p_{p} \equiv$ $\left|\boldsymbol{p}_{p}\right|$. The distorted spectral function includes the IA, FSI, and $\mathrm{FSI}^{2}$ terms.

transfer a momentum of the order $p_{p} \sim 100 \mathrm{MeV}$ to the spectator proton is by pushing it sideways. At larger recoil momenta $p_{p} \gtrsim 300 \mathrm{MeV}$ it becomes increasingly possible for the DIS hadron to push the spectator forward, resulting in an enhancement of the spectral function in the forward region. In contrast, the backward region is protected from this effect, as it is kinematically impossible for the forward-moving DIS hadron to push the spectator backwards.

The results shown in Figs. 13 and 14 are close to those obtained in Ref. [46] for the distorted spectral function of quasielastic deuteron breakup $d\left(e, e^{\prime} p\right) n$ at intermediate energies $\sim$ few $\mathrm{GeV}$ in the Glauber approximation. This is natural, as our distorted spectral function also describes quasielastic breakup, if the tagged DIS structure function of the nucleon is replaced by its elastic structure function, i.e., by the square of the nucleon elastic form factor at the corresponding momentum transfer $Q^{2}$.

Figure 15 shows the distorted spectral function as a function of the invariant momentum transfer $t^{\prime}$ and the recoil LF fraction $\alpha_{p}$, as used in neutron structure extraction and on-shell extrapolation. The plot again shows the ratio $S_{d}[\mathrm{dist}] / S_{d}[\mathrm{IA}]$, Eqs. (6.52)-(6.55), and gives separately the results for the IA, the sum IA + FSI, and the sum IA + FSI + FSI ${ }^{2}$ (total). One sees the following:

(i) For $\left|t^{\prime}\right| \lesssim 0.1 \mathrm{GeV}^{2}$ the correction arises mainly from the linear FSI term and is negative.

(ii) For $\left|t^{\prime}\right| \gtrsim 0.2 \mathrm{GeV}^{2}$ the $\mathrm{FSI}^{2}$ term dominates and causes a steep rise of the ratio.

(iii) The distorted spectral function is again positive for all $t^{\prime}$.

The approximations made in our calculation of FSI are reliable at moderate recoil momenta $\left|\boldsymbol{p}_{p}\right| \lesssim 200 \mathrm{MeV}$, or $\left|t^{\prime}\right| \lesssim$ 


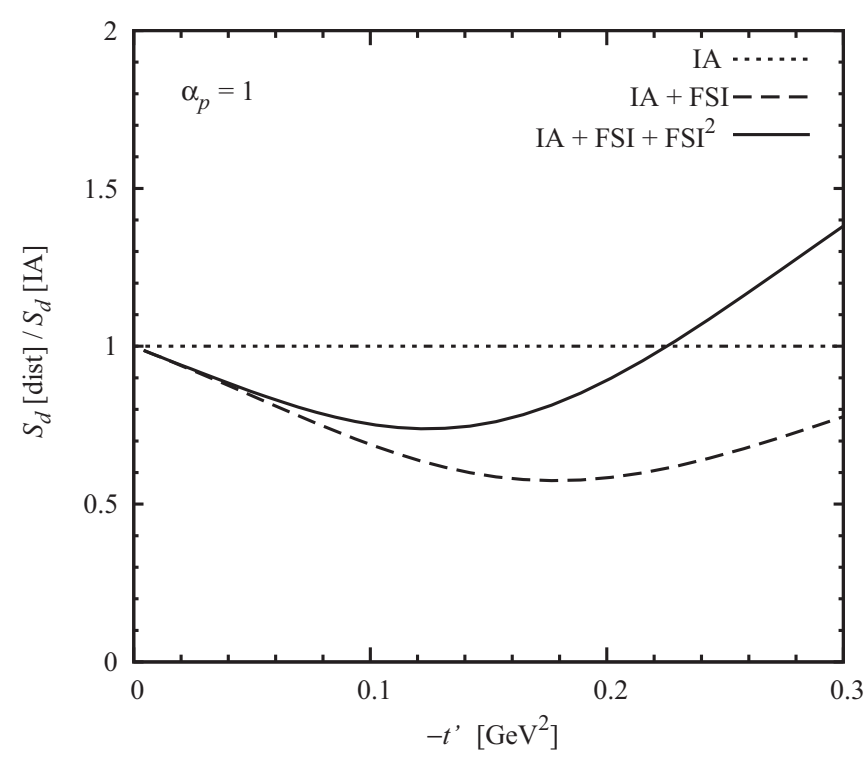

FIG. 15. The ratio of the distorted spectral function to the IA spectral function, $S_{d}$ [dist] $/ S_{d}[\mathrm{IA}]$, Eqs. (6.52)-(6.55), as a function of $-t^{\prime}$, for a fixed value $\alpha_{p}=1$. The plot shows separately the IA, $\mathrm{IA}+\mathrm{FSI}$, and IA + FSI + FSI $^{2}$ results.

$0.1 \mathrm{GeV}^{2}$, where the dominant correction comes from the $S$-wave IA interfering with the $S$-wave rescattering integral (see Sec. VID). The results for higher momenta in Figs. 14 and 15 are estimates and shown for illustration only. Our objectives in the present study are to calculate the dominant linear FSI correction at low recoil momenta, and to estimate roughly at what recoil momenta the quadratic FSI correction becomes comparable to the linear one. This information is sufficient for assessing the prospects for neutron structure extraction from future tagged DIS measurements with EIC (see below). An accurate description of FSIs in tagged DIS at recoil momenta $\left|\boldsymbol{p}_{p}\right| \gtrsim 300 \mathrm{MeV}$ would require more accurate construction of the deuteron LF wave function at large relative momenta, as well as more detailed modeling of the slow hadron distribution and the rescattering process.

\section{G. Analytic properties}

We must also investigate the effect of FSIs on the analytic properties of the spectral function in $t^{\prime}$. The IA current matrix element contains the nucleon pole of the deuteron wave function at $t^{\prime}=0$, which causes the IA spectral function to behave as $\sim R /\left(t^{\prime}\right)^{2}$ in the limit $t^{\prime} \rightarrow 0$ (see Sec. IV G). It is easy to see that the FSI contribution to the current matrix element is nonsingular in the limit $t^{\prime} \rightarrow 0$. This follows from the fact that the rescattering integral $I_{d}$, Eq. (6.20), or the corresponding nonrelativistic approximation Eq. (6.50), is a smooth function of the recoil momentum in the physical region $\left|\boldsymbol{p}_{p}(\mathrm{RF})\right|>0$ (or $t^{\prime}<t_{0}^{\prime}$ ), remains regular at $\left|\boldsymbol{p}_{p}(\mathrm{RF})\right|=0$, and can thus be continued to the unphysical point $\left|\boldsymbol{p}_{p}(\mathrm{RF})\right|^{2}=t_{0}^{\prime} / 2=$ $-M_{N} \epsilon_{d}+\epsilon^{2} / 4$ (or $t^{\prime}=0$ ) without encountering singularities of the deuteron wave function. In the invariant formulation using Feynman graphs, it follows from the fact that the nucleon pole arises from the nucleon tree graph, while the loop graphs

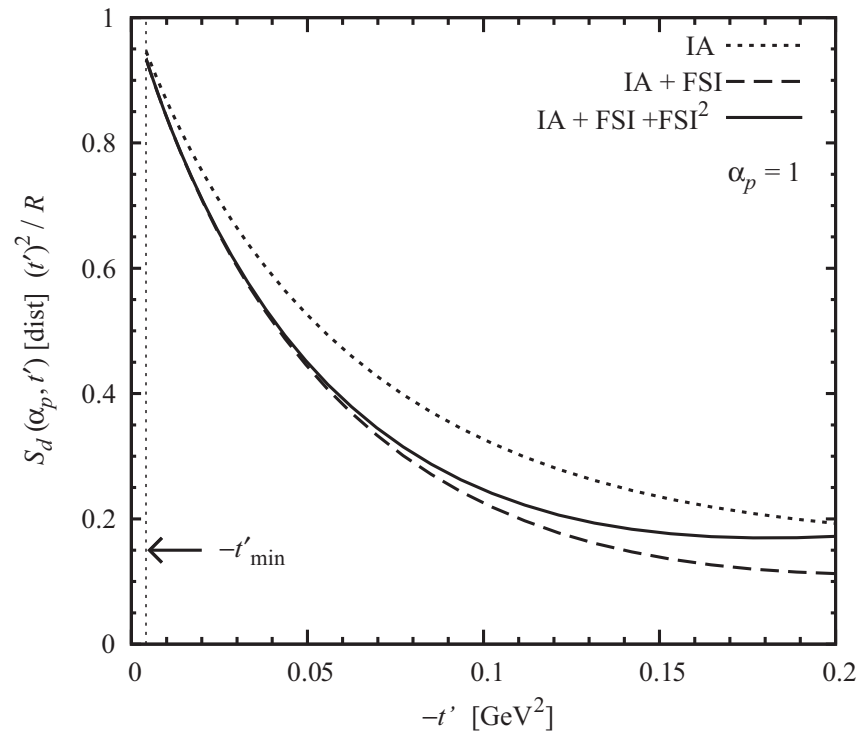

FIG. 16. The distorted spectral function $S_{d}$ [dist], Eqs. (6.52)(6.55), with the pole factor $R /\left(t^{\prime}\right)^{2}$ extracted, as function of $t^{\prime}$, for $\alpha_{p}=1$ (cf. Fig. 7). The plot shows separately the IA, IA + FSI, and $\mathrm{IA}+\mathrm{FSI}+\mathrm{FSI}^{2}$ results.

describing FSIs can at most modify the subleading behavior. A formal proof of this "no-loop theorem" was given in Ref. [15].

Figure 16 shows the distorted deuteron spectral function with the pole factor removed. The plot shows separately the IA, the sum IA + FSI, and the sum IA + FSI + FSI $^{2}$ (total). One sees the following:

(i) The FSI correction vanishes when approaching the pole.

(ii) The $\mathrm{FSI}^{2}$ correction vanishes even faster than the FSI one.

(iii) The magnitude of the FSI correction is $\sim 30 \%$ of the IA at $\left|t^{\prime}\right| \sim 0.1 \mathrm{GeV}^{2}$ and decreases approximately linearly in $\left|t^{\prime}\right|$ as $\left|t^{\prime}\right| \rightarrow 0$.

The fact that FSI does not modify the nucleon pole singularity of the IA spectral function is of central importance for the extraction of neutron structure from DIS on the deuteron with proton tagging. It implies that FSIs can be eliminated in a model-independent manner through the on-shell extrapolation procedure described in Sec. IV G. The FSI modifies the measured tagged structure at $t^{\prime}<t_{0}^{\prime}$ but drops out when performing the extrapolation to $t^{\prime}=0[15]$.

\section{H. Sum rules and unitarity}

Important physical requirements of the deuteron spectral function are the nucleon number and LF momentum sum rules, Eqs. (4.21) and (4.22). They express the fact that the initial state consists of two nucleons and does not involve nonnucleonic degrees of freedom. The interactions summarized by the deuteron wave function distribute the LF momentum among the two nucleons but do not change the baryon number or the overall LF momentum of the system. The IA result for the spectral function satisfies both sum rules, and we would like them to be satisfied in the presence of FSI as well. We want to comment briefly how this is realized within our model of FSIs. 
The nucleon number sum rule Eq. (4.21) demands that the integral of the distorted spectral function over the recoil proton momentum $\left(\alpha_{p}, \boldsymbol{p}_{p T}\right)$ satisfy

$$
\begin{aligned}
& \int \frac{d \alpha_{p}}{\alpha_{p}} \int d^{2} p_{p T} S_{d}\left(\alpha_{p}, \boldsymbol{p}_{p T}\right)[\text { dist }] \\
& =2(2 \pi)^{3} \int d \Gamma_{p} S_{d}\left(\alpha_{p}, \boldsymbol{p}_{p T}\right)[\text { dist }]=1 .
\end{aligned}
$$

Since the IA spectral function alone already satisfies the sum rule, Eq. (6.66) requires that the integral over the total FSI correction (linear and quadratic) be zero,

$$
\int d \Gamma_{p} S_{d}\left(\alpha_{p}, \boldsymbol{p}_{p T}\right)\left[\mathrm{FSI}+\mathrm{FSI}^{2}\right]=0 .
$$

Equations (6.66) viz. (6.67) are realized in our formulation if the scattering process between the slow hadron and the spectator proton is elastic, i.e., if it only redistributes the momentum between the particles but preserves the overall flux. This could be achieved by constructing an effective interaction such that the operator converting the plane-wave state into the scattering state [the time evolution operator of Eq. (6.9)] is explicitly unitary when restricted to the space of states with fixed energy, i.e., when considering the energy-conserving matrix elements only. How such an operator could be constructed explicitly is an interesting problem for further study.

With the present approximations we can describe the rescattering process quantitatively only at recoil momenta $|\boldsymbol{p}|_{p} \lesssim$ $300 \mathrm{MeV}$, which are not sufficient to verify the condition Eq. (6.67) explicitly. However, we can at least observe the tendencies that could lead to the condition Eq. (6.67) being satisfied in a more complete treatment. At small recoil momenta $|\boldsymbol{p}|_{p} \lesssim 300 \mathrm{MeV}$ the linear FSI term dominates and contributes negatively to Eq. (6.67), while at larger momenta $|\boldsymbol{p}|_{p}>$ $300 \mathrm{MeV}$ the quadratic FSI term takes over and contributes positively (see Fig. 14). It is thus apparent how Eq. (6.67) could be satisfied by way of cancellation between the lowand high-momentum regions, even if we presently cannot compute the high-momentum contributions accurately within our scheme. In this sense we have focused on modeling the low-momentum rescattering using empirical interactions, and trust that Eq. (6.67) will be restored by high-momentum contributions outside of the present approximations.

The LF momentum sum rule Eq. (4.22) follows from the nucleon number sum rule Eq. (4.21) if the function $\left(2-\alpha_{p}\right) S_{d}\left(\alpha_{p}, \boldsymbol{p}_{p T}\right)$ is symmetric under $\alpha_{p} \rightarrow 2-\alpha_{p}$, which amounts to interchange of the LF momenta of the active nucleon and the spectator. The IA result embodies this symmetry exactly thanks to the symmetry of the deuteron LF wave function. The FSI correction does not satisfy it exactly, as the rescattering integral $I_{d}$ in Eqs. (6.29) and (6.30) is generally not symmetric under $\alpha_{p} \rightarrow 2-\alpha_{p}$. However, the symmetry of the spectral function is still approximately realized within our scheme, as the variation of $I_{d}$ in $\alpha_{p}$ around $\alpha_{p}=1$ is much slower than that of $\psi_{d}$, so that $I_{d}$ can effectively be regarded as a constant for the purpose of the reflection symmetry, and the symmetry of Eqs. (6.29) is again brought about by that of the deuteron wave function. In this sense also the momentum sum rule of the spectral function is preserved by the FSI within our scheme of approximations. Note that our physical picture of FSIs applies only in a limited range of $x$, so that it is not possible to test the momentum sum rule for the deuteron structure function, Eq. (4.29), within our model.

\section{NEUTRON STRUCTURE EXTRACTION}

Our findings regarding the momentum and angular dependence of FSIs have implications for the extraction of neutron structure functions from deuteron DIS data with proton tagging. A full assessment of the strategy requires an estimate of the uncertainties of the tagged structure function measurements and should be made with realistic pseudodata. Nevertheless, some general conclusions can be drawn already at this level.

The preferred method for extracting the free neutron structure function is the on-shell extrapolation in $t^{\prime}$ at fixed $\alpha_{p}$ (see Sec. IV G). The procedure eliminates modifications due to nuclear binding as well as FSIs. The accuracy of the extrapolation depends on several factors: (a) the uncertainties of the tagged structure function data; (b) the smoothness of the $t^{\prime}$ dependence of the spectral function after removing the pole factor, which is determined by the FSI; (c) the distance between the physical region and the on-shell point, which depends on the recoil fraction $\alpha_{p}$.

If accurate measurements of the tagged structure functions can be made down to rest-frame recoil momenta $p_{p}(\mathrm{RF}) \sim$ few $10 \mathrm{MeV}$, one may perform the on-shell extrapolation in $t^{\prime}$ at LF fractions $\alpha_{p} \approx 1$, where one can come closest to the pole in $t^{\prime}$ (see Fig. 4). In this situation our model predicts that the FSI corrections have smooth $t^{\prime}$ dependence, and a magnitude of $<10 \%$ of the IA at the lowest $t^{\prime}$ values, so that they are reliably eliminated by the extrapolation procedure. Since there are no singularities in $t^{\prime}$ between $t_{0}^{\prime}$ and 0 the extrapolation can be performed using a polynomial fit to the $t^{\prime}$ dependence of the tagged structure function data [15].

If accurate measurements of the tagged structure functions are only possible at larger recoil momenta $p_{p}(\mathrm{RF}) \sim 100-200$ $\mathrm{MeV}$, one may instead focus on the backward region $\alpha_{p}>1$ (or $\cos \theta_{p q}<0$ ), where our model predicts that FSIs are relatively small; see Figs. 13 and 14. In this case there is a trade-off between coming as close as possible to the pole (which favors $\alpha_{p}=1$ ) and minimizing FSIs (which favors $\alpha_{p}$ substantially larger than 1). While our model predicts that the $t^{\prime}$ dependence is smooth even in the presence of FSIs, the distances from the pole are such that the magnitude of the correction is substantial ( $\sim 30 \%$ of the IA at $\left|t^{\prime}\right|=0.1 \mathrm{GeV}^{2}$; see Fig. 16). In this situation one may no longer rely on polynomial extrapolation but fit the data with a more complex parametrization of the spectral function based on the expected functional form of the FSI correction.

\section{SUMMARY AND OUTLOOK}

In this work we have presented a theoretical framework for the analysis of DIS on the deuteron with spectator nucleon tagging. Nuclear and nucleonic structure are separated using the apparatus of LF quantum mechanics appropriate for high-energy scattering processes. The IA determines the basic dependence of the tagged cross section on the recoil momentum and its analytic properties (nucleon pole). In the 
region of intermediate $x$ (roughly $0.1<x<0.5$ ) FSIs arise mainly from the interaction of the spectator with slow hadrons produced in the fragmentation of the active nucleon (rest frame momenta $\left|\boldsymbol{p}_{h}\right| \sim 1 \mathrm{GeV}$ ). We have described this effect in a schematic model using empirical slow-hadron distributions (protons, neutrons) and the nucleon-nucleon scattering amplitude. The main conclusions regarding FSI in tagged DIS can be summarized as follows:

(i) Absorption and refraction. The rescattering between the slow DIS hadrons and the spectator nucleon involves absorptive interactions (linear in the imaginary part of amplitude, dominant at recoil momenta $|\boldsymbol{p}|_{p}<200 \mathrm{MeV}$ ) and refractive interactions (quadratic in real and imaginary parts, dominant at higher momenta). The net effect is to reduce the flux of spectator nucleons at low momenta and increase it at higher momenta.

(ii) Angular dependence. The FSI effect on the recoil momentum distribution is approximately isotropic in the deuteron rest frame at momenta $\left|\boldsymbol{p}_{p}\right|<100 \mathrm{MeV}$. At higher momenta it becomes angle dependent, with the strongest effect occurring in the direction approximately perpendicular to the $\boldsymbol{q}$-vector (sideways direction).

(iii) Analyticity in $t^{\prime}$. The FSI correction to the IA spectral function vanishes at the nucleon pole $t^{\prime} \rightarrow 0$ (relative to the IA) and exhibits a smooth dependence on $t^{\prime}$ up to $\left|t^{\prime}\right| \sim 0.1 \mathrm{GeV}^{2}$. It can be eliminated through on-shell extrapolation $t^{\prime} \rightarrow 0$.

(iv) Relative magnitude. The FSI correction reduces the IA spectral function by $\sim 30 \%$ at $\left|t^{\prime}\right| \sim 0.1 \mathrm{GeV}^{2}$ and $\alpha_{p}=1$. The effect becomes proportionately smaller as $\left|t^{\prime}\right|$ decreases.

Our results show that extraction of free neutron structure through on-shell extrapolation is possible if accurate measurements of the recoil momentum dependence can be performed in the region $\left|t^{\prime}\right| \ll 0.1 \mathrm{GeV}^{2}$ (or $\left|\boldsymbol{p}_{p}\right| \ll 200 \mathrm{MeV}$ ). The analytic structure of the FSI correction and its moderate size indicate that the nucleon pole residue can be extracted reliably even in the presence of experimental errors. We emphasize that the extrapolation eliminates not only Fermi motion but also nuclear binding effects, as the phase space for interactions vanishes at the on-shell point. Measurements of tagged DIS over a wide kinematic range will become possible at a future EIC with suitable forward detectors. Simulations of neutron structure extraction through on-shell extrapolation using the IA cross section model suggest that the procedure is feasible under realistic conditions $[79,80]$. The dominant systematic uncertainty in the tagged structure function results from the uncertainty in the transverse recoil momentum $\boldsymbol{p}_{p T}$, as caused by the finite detector resolution and the intrinsic momentum spread of the deuteron beam [81]. These simulations can now be updated to include FSI effects in the cross section model; results will be reported elsewhere.

Tagged DIS on the deuteron has also been proposed as a tool to explore the dynamical origin of the nuclear modification of the nucleon's partonic structure. The idea is that the observed recoil momentum effectively controls the spatial size of the $p n$ configuration in the deuteron, which makes is possible to study nuclear modifications of the nucleon structure functions in configurations of defined size ("tagged EMC effect") $[1,45,82,83]$. The main challenge in such measurements is to separate initial-state modifications of the partonic structure from FSI effects. Our model provides an a priori estimate of the size of the FSI effect and can be used to assess the sensitivity of such measurements to a putative nuclear modification of nucleon structure. In particular, the results of Fig. 14 show that in the backward region $\cos \theta_{p q}<-0.7$ the FSI effect is practically independent of the modulus of the recoil momentum for values $\left|\boldsymbol{p}_{p}\right| \gtrsim 300 \mathrm{MeV}$. An observed variation of the tagged structure function with $\left|\boldsymbol{p}_{p}\right|$ in this region could therefore be ascribed to initial-state modifications. The formulation of a practical procedure for tagged EMC effect studies at the EIC based on these findings should be the object of future work.

In applications to neutron structure and the EMC effect one aims to eliminate or minimize the FSI effects in tagged DIS. The same measurements could be used to study the FSI as an object in itself, by going to kinematics where the effects are maximal (recoil angles $-0.2<\cos \theta_{p q}<0.4$; see Fig. 14) and verifying their strong kinematic dependence. Such measurements on the deuteron would help us to understand better the pattern of nuclear breakup in DIS on heavier nuclei (e.g., slow neutron rates and angular distributions), which in turn would assist other studies of hard processes in nuclei (centrality dependence, hadronization and jets in nuclei) [5].

In the present study of FSIs in tagged DIS we considered the case of unpolarized electron-deuteron scattering and made several simplifying assumptions about the DIS hadron spectrum and the rescattering process. The treatment could be refined in several aspects while remaining within the same physical picture:

(i) Spin effects in hadron-spectator rescattering. Absent experimental data or theoretical arguments suggesting otherwise, we have modeled the slow hadrons produced in the DIS process as an unpolarized beam. Correspondingly, we have neglected the spin dependence of the hadron-proton rescattering process. This has allowed us to compute the dominant FSI effect at recoil momenta $p_{p}<200 \mathrm{MeV}$, resulting from the $S$-wave IA amplitude interfering with the $S$-wave FSI amplitude. Spin dependence of the rescattering could be included if data about the effective polarization of the slow hadrons become available. Spin effects could also arise without slow hadron polarization, from a spin-orbit term in the hadron-proton rescattering amplitude. Both effects could give rise to $S$ - $D$ wave interference in the distorted spectral function. Such interference terms are not expected to significantly change the results compared to the dominant diagonal $S$-wave contribution at small recoil momenta. In particular, the on-shell extrapolation should not be affected.

(ii) Polarized electron-deuteron DIS. The methods developed here could be extended to the case of polarized electron scattering from a polarized deuteron, including possible tensor polarization of the spin-1 system. The basic formalism for the LF description of the polarized deuteron in DIS is given in Ref. [41]. A detailed treatment of polarized deuteron LF structure and double-polarized tagged DIS in the IA will be presented in Ref. [40]. The formalism for FSIs developed here could be applied to the polarized case without essential changes. In this case one should allow also for spin dependence of the slow hadron distribution (correlated with the spin of the active neutron), and of the the rescattering process. An interesting aspect of polarized tagged DIS is the appearance 
of time-reversal-odd ( $T$-odd) response functions in the cross section, which are zero in the IA and become nonzero only due to FSIs, and which therefore provide sensitive tests of the FSI dynamics.

(iii) Inelastic rescattering. In the present calculation we implement FSIs through elastic rescattering of slow hadrons (protons, neutrons) on the spectator. This scheme allows us to describe FSIs in the hadronic tensor at the probabilistic level (because the same hadrons appear in the current matrix element with and without FSIs) and preserve the nucleon number sum rule through elastic unitarity. It is clear that certain inelastic channels are open at the momenta $\left|\boldsymbol{p}_{h}\right| \sim 1 \mathrm{GeV}$ considered here and can have sizable cross section, for example, production of $\Delta$ isobars in nucleon-nucleon collisions. Including such channels in the FSI calculation is possible in theory but very difficult in practice. It requires a coupled-channel formalism, in which one considers all stable hadrons appearing in the final state (in the example of $\Delta$ excitation, this would be two nucleons and one pion) and implements all possible interactions between them. One would also need to know the amplitudes for the "direct" production of these hadrons through fragmentation of the active nucleon, which interfere with those of "indirect" production in the rescattering process. It is not obvious whether these amplitudes could be extracted from the DIS hadron multiplicities without further modeling.

(iv) Rescattering of pions. We have focused here on FSIs induced by the rescattering of slow protons and neutrons in the DIS final state, as these are the dominant hadrons at $x_{\mathrm{F}}<-0.3$ and have large cross sections for rescattering on the spectator nucleon. FSIs could also arise from the rescattering of pions, whose multiplicity rises strongly at $x_{\mathrm{F}}>-0.3$. This effect could be calculated with the same formalism as used here (the formulas for the rescattering integral in Appendix D are given for a general slow hadron mass). The pion-nucleon amplitude at pion momenta $\left|\boldsymbol{p}_{h}\right| \sim 1 \mathrm{GeV}$ is well constrained by data. One interesting aspect of pions is that they can emerge in the backward direction of the DIS process, i.e., opposite to the $\boldsymbol{q}$-vector [see Sec. V A and Eq. (5.5)], and therefore push the spectator in the backward direction. It would be worth investigating pion-induced FSIs in a separate study.

The physical picture of FSIs developed here refers to the kinematic region of intermediate $x$, roughly $0.1 \lesssim x \lesssim 0.5$. In this region the slow hadron distributions extend over a broad range of $\mathrm{LF}$ fractions $\zeta_{h}<1-\xi \approx 1-x$, while diffractive hadron production is not yet important. Tagged DIS experiments can of course be performed also at larger or smaller values of $x$, with various scientific objectives. It is worthwhile summarizing what changes in the physical picture of FSIs are expected in these regions.

(i) FSI and diffraction at small $x$. At $x \ll 0.1$ diffractive DIS becomes a distinctive source of slow nucleons in the target fragmentation region. The $x_{\mathrm{F}}$ spectra of protons in DIS on the proton measured at HERA show a diffractive peak near $x_{\mathrm{F}}=-1$ with an integrated multiplicity of $\sim 0.1$; see Ref. [84] for a review. Physically this effect is explained by a color-singlet exchange between the electromagnetic current and the nucleon, such that the DIS process leaves the nucleon intact and recoiling with a momentum $\sim$ few $100 \mathrm{MeV}$. If such diffractive production happens in tagged DIS on the deuteron, there is a significant probability for the diffractive nucleon and the spectator to recombine and form the deuteron, as they have the same spin-isospin quantum numbers and similar momenta as the original proton-neutron pair in the deuteron wave function. In measurements of tagged DIS at small $x$ one selects the channel where this recombination does not happen and a proton-neutron scattering state is produced instead of the deuteron. In this situation it is essential that the wave function of the scattering state is constructed such that it is orthogonal to the deuteron, i.e., that it is obtained as the solution of the dynamical equation with the same effective interaction as produces the deuteron bound state, $\mathrm{cf}$. Eq. (6.8). It also requires that off-shell energies are allowed in the rescattering process. A detailed treatment of FSIs in tagged DIS at small $x$ will be presented in a forthcoming article [22].

(ii) FSIs in tagged DIS at large $x$. In DIS on the nucleon at $x \gtrsim 0.5$ the distribution of hadrons in the target fragmentation region differs substantially from that at lower $x$. The reason is that the hadron LF fraction is kinematically restricted to $\zeta_{h}<1-\xi \approx 1-x$, such that only small values of $\zeta_{h}$ are allowed at $x \rightarrow 1$. Physically speaking the DIS process almost "empties" the nucleon of LF momentum, and the produced hadrons have to share the small rest. These hadrons therefore have large momenta in the target rest frame, cf. Eq. (5.5) and Figs. 9 and 10, and their interactions with the spectator are suppressed by the formation time. Our picture therefore suggests that FSIs may be suppressed in tagged DIS at large $x$. However, since $F_{2 n} \ll F_{2 p}$ at $x \rightarrow 1$, even small FSIs would have a large relative effect on the extracted neutron structure function. The $x \rightarrow 1$ limit of tagged DIS therefore requires a dedicated study.

\section{ACKNOWLEDGMENTS}

This work was motivated by Jefferson Lab's 2014-2015 Laboratory-directed R\&D project "Physics potential of polarized light ions with EIC@JLab" (see Ref. [81]). We are indebted to W. Cosyn, V. Guzey, D. Higinbotham, Ch. Hyde, P. Nadel-Turonski, K. Park, and M. Sargsian, for numerous discussions of theoretical and experimental aspects of nuclear DIS with spectator tagging. We thank R. Schiavilla for helpful communications regarding scattering theory and FSIs in deuteron breakup. This material is based upon work supported by the US Department of Energy, Office of Science, Office of Nuclear Physics, under Contract No. DE-AC05-06OR23177. The research of M.S. was supported by the US Department of Energy, Office of Science, Office of Nuclear Physics, under Award No. DE-FG02-93ER40771.

\section{APPENDIX A: DEUTERON WAVE FUNCTION}

In this Appendix we summarize the properties of the nonrelativistic deuteron wave function and its spin structure, as used in the present calculation. The nonrelativistic wave function in the rest frame is denoted by

$$
\widetilde{\Psi}_{d}\left(\boldsymbol{p}, \sigma_{p}, \sigma_{n} \mid \lambda_{d}\right),
$$

where $\lambda_{d}=( \pm 1,0)$ is the deuteron spin projection, $\boldsymbol{p}$ is the proton momentum, and $\sigma_{p, n}= \pm \frac{1}{2}$ are the neutron and proton 
spin projections. The wave function is normalized such that

$$
\sum_{\sigma_{p}, \sigma_{n}} \int d^{3} p \widetilde{\Psi}_{d}^{*}\left(\boldsymbol{p}, \sigma_{p}, \sigma_{n} \mid \lambda_{d}^{\prime}\right) \widetilde{\Psi}_{d}\left(\boldsymbol{p}, \sigma_{p}, \sigma_{n} \mid \lambda_{d}\right)=\delta\left(\lambda_{d}^{\prime}, \lambda_{d}\right) .
$$

It can be represented as a bilinear form in nucleon spinors as

$$
\begin{aligned}
& \widetilde{\Psi}_{d}\left(\boldsymbol{p}, \sigma_{p}, \sigma_{n} \mid \lambda_{d}\right) \\
& =\frac{1}{\sqrt{2}} \epsilon^{i}\left(\lambda_{d}\right)\left[\delta^{i j} U(p)+\frac{1}{\sqrt{2}}\left(\frac{3 p^{i} p^{j}}{p^{2}}-\delta^{i j}\right) W(p)\right] \\
& \quad \times \chi^{\dagger}\left(\sigma_{n}\right) \sigma^{j}\left(i \sigma^{2}\right) \chi\left(\sigma_{p}\right),
\end{aligned}
$$

where $\boldsymbol{\epsilon}\left(\lambda_{d}\right)$ are the polarization vectors describing the spin states of the spin-1 deuteron,

$$
\begin{aligned}
\boldsymbol{\epsilon}( \pm 1) & =\frac{1}{\sqrt{2}}\left(\begin{array}{r}
\mp 1 \\
-i \\
0
\end{array}\right), \quad \boldsymbol{\epsilon}(0)=\left(\begin{array}{l}
0 \\
0 \\
1
\end{array}\right), \quad(\mathrm{A}) \\
\sum_{\lambda_{d}} \epsilon^{i}\left(\lambda_{d}\right) \epsilon^{j *}\left(\lambda_{d}\right) & =\delta^{i j}, \quad \epsilon^{*}\left(\lambda_{d}^{\prime}\right) \cdot \boldsymbol{\epsilon}\left(\lambda_{d}\right)=\delta\left(\lambda_{d}^{\prime}, \lambda_{d}\right),
\end{aligned}
$$

and $\chi\left(\sigma_{n, p}\right)$ are the spinors describing the spin states of the spin- $\frac{1}{2}$ nucleons,

$$
\begin{aligned}
\chi\left(\frac{1}{2}\right) & =\left(\begin{array}{l}
1 \\
0
\end{array}\right), \quad \chi\left(-\frac{1}{2}\right)=\left(\begin{array}{l}
0 \\
1
\end{array}\right), \\
\sum_{\sigma} \chi^{\alpha}(\sigma) \chi^{\beta *}(\sigma) & =\delta^{\alpha \beta}, \quad \chi\left(\sigma^{\prime}\right) \chi^{\dagger}(\sigma)=\delta\left(\sigma^{\prime}, \sigma\right) .
\end{aligned}
$$

In the bilinear form in Eq. (A3), $\sigma^{j}$ are the Pauli spin matrices, and $i \sigma^{2}$ is the metric spinor relating the contravariant and covariant spinor components. $U(p)$ and $W(p)$ are the radial wave functions of the $S$ and $D$ waves (angular momentum $L=0$ and 2) and are normalized such that

$$
4 \pi \int_{0}^{\infty} d p p^{2}\left[U^{2}(p)+W^{2}(p)\right]=1
$$

The unpolarized nucleon density matrix in the deuteron (averaged over the deuteron spin, and summed over the neutron and proton spins) is

$$
\begin{aligned}
& \rho_{d}\left(\boldsymbol{p}_{2}, \boldsymbol{p}_{1}\right) \\
& \quad \equiv \frac{1}{3} \sum_{\lambda_{d}} \sum_{\sigma_{p}, \sigma_{n}} \widetilde{\Psi}_{d}\left(\boldsymbol{p}_{2}, \sigma_{p}, \sigma_{n} \mid \lambda_{d}\right) \widetilde{\Psi}_{d}^{*}\left(\boldsymbol{p}_{1}, \sigma_{p}, \sigma_{n} \mid \lambda_{d}\right) \\
& =U\left(p_{2}\right) U\left(p_{1}\right)+\frac{1}{2}\left[\frac{3\left(\boldsymbol{p}_{2} \boldsymbol{p}_{1}\right)^{2}}{p_{2}^{2} p_{1}^{2}}-1\right] W\left(p_{2}\right) W\left(p_{1}\right) .
\end{aligned}
$$

Note that the density matrix is diagonal in $S$ and $D$ waves (no mixing) even in the case of unequal momenta $\boldsymbol{p}_{2} \neq \boldsymbol{p}_{1}$. The diagonal density matrix, describing the probability to find a nucleon with given momentum $\boldsymbol{p}$, is

$$
\rho_{d}(\boldsymbol{p}, \boldsymbol{p})=U^{2}(p)+W^{2}(p), \quad \int d^{3} p \rho_{d}(\boldsymbol{p}, \boldsymbol{p})=1 .
$$

Figure 17 shows the integral of the momentum density over finite intervals $p_{1}<|\boldsymbol{p}|<p_{2}$, corresponding to the probabil-

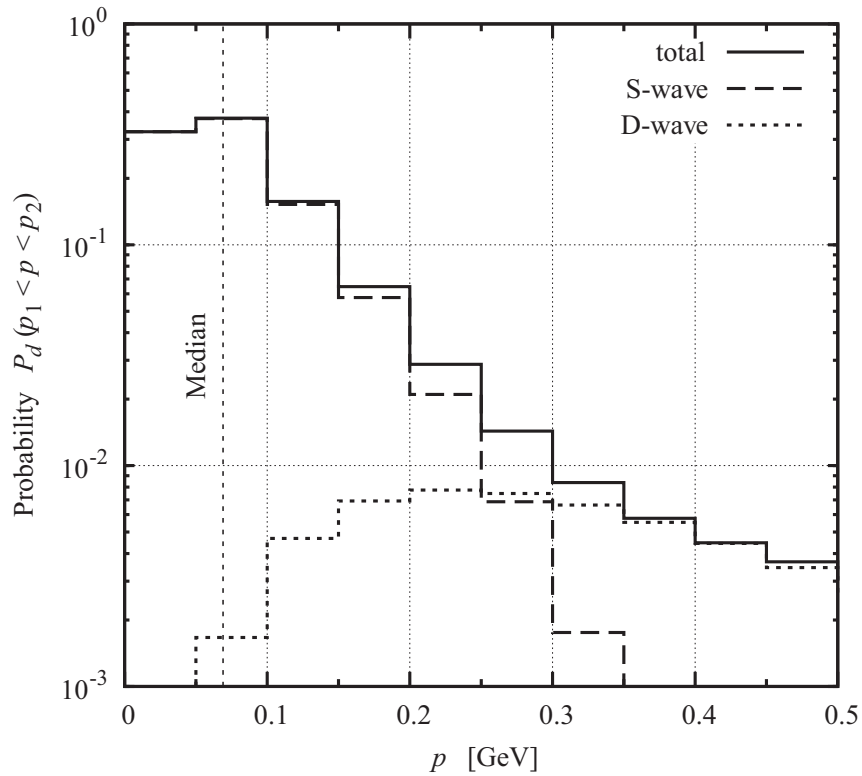

FIG. 17. Momentum distribution of the deuteron with the AV18 wave function [56]. The histogram shows the probabilities to find a nucleon with momentum $p_{1}<p<p_{2}$, Eq. (A12), for $p_{1,2}$ in steps of $50 \mathrm{MeV}$. The constant values shown at $p_{1}<p<p_{2}$ give the value of $P_{d}\left(p_{1}<p<p_{2}\right)$ for that range. The median momentum is indicated by a vertical line.

ity to find a nucleon with momentum in that range,

$$
\begin{aligned}
P_{d}\left(p_{1}<|\boldsymbol{p}|<p_{2}\right) & =\int d^{3} p \theta\left(p_{1}<|\boldsymbol{p}|<p_{2}\right) \rho_{d}(\boldsymbol{p}, \boldsymbol{p}) \\
& =4 \pi \int_{p_{1}}^{p_{2}} d p p^{2}\left[U^{2}(p)+W^{2}(p)\right] .
\end{aligned}
$$

The histogram gives an intuitive picture of the momentum distribution of nucleons in the deuteron and enables simple estimates of the contribution of different momentum regions to observables. The median of the momentum distribution is $69 \mathrm{MeV}$ for the AV18 wave function. Note that the median nucleon momentum in the deuteron is considerably larger than the "binding momentum" defined as $\sqrt{M_{N} \epsilon_{d}}=45 \mathrm{MeV}$; the different values illustrate the presence of multiple dynamical scales in the deuteron wave function.

\section{APPENDIX B: PROJECTION FORMULAS}

In this Appendix we derive the explicit expressions of the proton-tagged deuteron structure functions in terms of the deuteron LF momentum density and the inclusive neutron structure functions, Eqs. (4.18) and (4.19), starting from the "master formula" for the scattering tensors in the collinear frame, Eq. (4.9). The same derivation can be used with the distorted spectral function in the presence of FSIs. We write 
Eq. (4.9) in schematic form as

$$
\begin{aligned}
W_{d}^{\mu \nu}\left(p_{d}, q, p_{p}\right) & =[\ldots] W_{n}^{\mu \nu}\left(p_{n}, \widetilde{q}\right), \\
{[\ldots] } & \equiv\left[2(2 \pi)^{3}\right] \frac{2\left|\Psi_{d}\left(\alpha_{p}, \boldsymbol{p}_{p T}\right)\right|^{2}}{\left(2-\alpha_{p}\right)^{2}},
\end{aligned}
$$

and substitute the deuteron tensor parametrized by Eq. (2.22), and the neutron tensor parametrized by Eq. (4.14). We consider the deuteron tensor averaged over the transverse direction of the recoil momentum, in which only the structures with $F_{L d}$ and $F_{T d}$ are present. Equations for the structure functions can be obtained by taking specific components of the tensor equation Eq. (B1) in the collinear frame (see Sec. IIC). From the longitudinal component $\mu \nu=++$ we obtain

$$
\begin{aligned}
F_{L d} & =[\ldots] \frac{L_{d}^{2}}{\left(L_{d}^{+}\right)^{2}}\left\{-\frac{\left(\widetilde{q}^{+}\right)^{2}}{\widetilde{q}^{2}} F_{L n}+\left[\frac{\left(\widetilde{L}_{n}^{+}\right)^{2}}{\widetilde{L}_{n}^{2}}+\frac{\left(\widetilde{q}^{+}\right)^{2}}{\widetilde{q}^{2}}\right] F_{T n}\right\} \\
& =[\ldots] \frac{L_{d}^{2}}{\left(L_{d}^{+}\right)^{2}}\left[\frac{\left(\widetilde{q}^{+}\right)^{2}}{\widetilde{q}^{2}}\left(F_{T n}-F_{L n}\right)+\frac{\left(\widetilde{L}_{n}^{+}\right)^{2}}{\widetilde{L}_{n}^{2}} F_{T n}\right] .
\end{aligned}
$$

From the transverse components $\mu \nu=i j,(i, j=x, y)$ we obtain

$$
F_{T d}-F_{L d}=[\ldots]\left[F_{T n}-F_{L n}+\frac{\left|\widetilde{\boldsymbol{L}}_{n T}\right|^{2}}{2 \widetilde{L}_{n}^{2}} F_{T n}\right] .
$$

Here we have used that, after averaging over the direction of the transverse recoil momentum, rotational symmetry allows us to replace

$$
\widetilde{L}_{n T}^{i} \widetilde{L}_{n T}^{j} \rightarrow \delta^{i j}\left|\widetilde{\boldsymbol{L}}_{n T}\right|^{2} / 2
$$

Explicit expressions for the structure functions are obtained from Eqs. (B3) and (B4) by substituting the specific expressions for the vector components in the collinear frame (see Sec. IIC). The deuteron vector $L_{d}$ is given by Eq. (2.38), and the nucleon vector $\tilde{L}_{n}$ is given by Eq. (4.16).

The exact expressions for the structure functions are complicated and not instructive. We quote only the expressions in the scaling limit $Q^{2} \gg M_{N}^{2},\left|\boldsymbol{p}_{p T}^{2}\right|$, where

$$
-\frac{L_{d}^{2}\left(\widetilde{q}^{+}\right)^{2}}{\left(L_{d}^{+}\right)^{2} \widetilde{q}^{2}} \rightarrow 1, \quad \frac{L_{d}^{2}\left(\widetilde{L}_{n}^{+}\right)^{2}}{\left(L_{d}^{+}\right)^{2} \widetilde{L}_{n}^{2}} \rightarrow 1, \quad \frac{\left|\widetilde{\boldsymbol{L}}_{n T}\right|^{2}}{2 \widetilde{L}_{n}^{2}}=O\left(\frac{\left|\boldsymbol{p}_{p T}\right|^{2}}{Q^{2}}\right) .
$$

In this limit the $L$ and $T$ deuteron structure functions are obtained as

$$
\begin{aligned}
& F_{L d}=[\ldots] F_{L n}, \\
& F_{T d}=[\ldots] F_{T n} .
\end{aligned}
$$

Reverting to the long form of Eq. (B1) and writing the arguments of the structure functions, this is

$$
\begin{aligned}
& F_{L d}\left(x, Q^{2}\right)=\left[2(2 \pi)^{3}\right] \frac{2\left|\Psi_{d}\left(\alpha_{p}, \boldsymbol{p}_{p T}\right)\right|^{2}}{\left(2-\alpha_{p}\right)^{2}} F_{L n}\left(\tilde{x}, Q^{2}\right), \\
& F_{T d}\left(x, Q^{2}\right)=\left[2(2 \pi)^{3}\right] \frac{2\left|\Psi_{d}\left(\alpha_{p}, \boldsymbol{p}_{p T}\right)\right|^{2}}{\left(2-\alpha_{p}\right)^{2}} F_{T n}\left(\widetilde{x}, Q^{2}\right) .
\end{aligned}
$$

The corresponding formula for the deuteron structure function $F_{2 d}=x_{d} F_{T d}$, Eq. (2.23), is

$$
F_{2 d}\left(x, Q^{2}\right)=\left[2(2 \pi)^{3}\right] \frac{\left|\Psi_{d}\left(\alpha_{p}, \boldsymbol{p}_{p T}\right)\right|^{2}}{2-\alpha_{p}} F_{2 n}\left(\tilde{x}, Q^{2}\right),
$$

where we have used that $x_{d}=x / 2, \tilde{x}=x /\left(2-\alpha_{p}\right)$, and $F_{2 n}\left(\tilde{x}, Q^{2}\right)=\tilde{x} F_{T n}\left(\widetilde{x}, Q^{2}\right)$.

\section{APPENDIX C: ELASTIC SCATTERING AMPLITUDE}

In this Appendix we give an empirical parametrization of the spin-averaged nucleon-nucleon elastic scattering amplitude at small angles and incident momenta $p \lesssim 1 \mathrm{GeV}$ (in the rest frame of the target nucleon), for use in our calculation of FSIs in tagged DIS on the deuteron.

Measurements of nucleon-nucleon elastic and total cross sections at incident momenta $p \sim 1 \mathrm{GeV}$ have been performed in several experiments; see Ref. [85] for a review of the data. Neutron-proton scattering measures directly the stronginteraction cross section; in proton-proton scattering one also has to account for electromagnetic interactions (Coulomb scattering) $[86,87]$. For both channels $(n p, p p)$ the differential strong-interaction cross section for elastic scattering at forward angles can be parametrized as

$$
\frac{d \sigma_{\mathrm{el}}}{d t}=|f(0)|^{2} e^{b t},
$$

where $f(t)$ is a complex amplitude and $b$ is the exponential slope. The amplitude is of the form

$$
f(t)=A(t)+\text { spin-dependent amplitudes, }
$$

where the central term $A(t)$ is nonzero at $t=0$ and can be expressed as

$$
A(0)=\operatorname{Im} A(0)\left(i+\rho_{0}\right), \quad \rho_{0} \equiv \operatorname{Re} A(0) / \operatorname{Im} A(0) .
$$

The imaginary part at $t=0$ is related to the nucleon-nucleon total cross section by the optical theorem

$$
[\operatorname{Im} A(0)]^{2}=\frac{\sigma_{\mathrm{tot}}^{2}}{16 \pi} .
$$

The contribution of spin-dependent amplitudes at $t=0$ can be described by the parameter

$$
\beta_{0}=\mid \text { spin-dependent amplitudes at } t=\left.0\right|^{2} /[\operatorname{Im} A(0)]^{2} .
$$

The differential cross section Eq. (C1) can then be represented as

$$
\frac{d \sigma_{\mathrm{el}}}{d t}=\frac{\sigma_{\mathrm{tot}}^{2}}{16 \pi}\left(1+\rho_{0}^{2}+\beta_{0}\right) e^{b t} .
$$

Experimental values of the parameters $\sigma_{\text {tot }}, \rho_{0}, \beta_{0}$, and $b$ at several energies are summarized in Table I. 
TABLE I. Parameters of the small-angle elastic scattering amplitude measured in $n p$ [86] and $p p$ [87] scattering experiments. Here $p$ is the momentum of the incident nucleon in the target rest frame (lab momentum), and $T=\sqrt{p^{2}+M_{N}^{2}}-M_{N}$ is the incident kinetic energy (lab energy).

\begin{tabular}{ccccrr}
\hline \hline & $p(\mathrm{GeV})$ & $T(\mathrm{GeV})$ & $\sigma_{\mathrm{tot}}(\mathrm{mb})$ & $\rho_{0}$ & $\beta_{0}$ \\
\hline$n p$ & 1.26 & 0.633 & 36.1 & -0.253 & $0.181 \pm 0.074$ \\
& 1.68 & 0.985 & $\sim 40$ & -0.414 & $<0.01$ \\
$p p$ & 1.28 & 0.648 & 41.1 & 0.202 & $0.096 \pm 0.030$ \\
& 1.69 & 0.992 & 47.5 & -0.178 & $0.025 \pm 0.012$ \\
\hline \hline
\end{tabular}

The differential cross section for nucleon-nucleon elastic scattering can be expressed in terms of the invariant scattering amplitude. Neglecting spin effects, we assume a single complex invariant amplitude $T(s, t)$ and write

$$
\frac{d \sigma_{\mathrm{el}}}{d t}=\frac{|T(s, t)|^{2}}{64 \pi s p_{\mathrm{cm}}^{2}}
$$

where $s$ is the squared CM energy and $p_{\mathrm{cm}}=\sqrt{s / 4-M_{N}^{2}}$ is the CM momentum. Comparing Eq. (C7) with the empirical formula Eq. (C6), we parametrize the invariant amplitude as

$$
\left.\begin{array}{rl}
|T(s, 0)|^{2}=4 s p_{\mathrm{cm}}^{2} \sigma_{\mathrm{tot}}^{2}\left(1+\rho_{0}^{2}+\beta_{0}\right) & |T(s, t)|=|T(s, 0)| e^{b t / 2} \\
\operatorname{Im} T(s, t)=\frac{|T(s, t)|}{\sqrt{1+\rho_{0}^{2}}}, & \operatorname{Re} T(s, t)=\frac{\rho_{0}|T(s, t)|}{\sqrt{1+\rho_{0}^{2}}}
\end{array}\right\} .
$$

These formulas apply at fixed $s$, and the parameters $\left(\sigma_{\mathrm{tot}}, \rho_{0}, \beta_{0}, b\right)$ generally depend on $s$. Equation (C8) can be adapted to the cases of $n p$ and $p p$ scattering by choosing appropriate parameters (cf. Table I) and provide a sufficient description of the nucleon-nucleon elastic amplitude for our purposes. For a simple parametrization of the average $n p$ and $p p$ amplitude we take the average of the parameter values at the lower energy of Table I,

$$
\sigma_{\mathrm{tot}}=39 \mathrm{mb}, \quad \rho_{0}=-0.03, \quad \beta_{0}=0.14, \quad b=4.3 \mathrm{GeV}^{-2} \text {. }
$$

For a more realistic parametrization one may use the energydependent parameters quoted in Ref. [85]. We note that the $\mathrm{Re} / \mathrm{Im}$ ratio of the amplitude, $\rho_{0}$, is poorly constrained by experimental data and relies on theoretical calculations.

\section{APPENDIX D: RESCATTERING INTEGRAL}

In this Appendix we evaluate the rescattering integral for the FSI correction in the form Eq. (6.50),

$$
\begin{aligned}
I_{d}\left(\boldsymbol{p}_{p}, \boldsymbol{p}_{h}\right)= & \int\left[d p_{p 1}\right]_{\text {shell }}[\ldots] \\
\equiv & \int \frac{d^{3} p_{p 1}}{(2 \pi)^{3} 2 E_{N}\left(\boldsymbol{p}_{p 1}\right)} \\
& \times 2 \pi \delta\left[\left(p_{p}+p_{h}-p_{p 1}\right)^{2}-M_{h}^{2}\right][\ldots],
\end{aligned}
$$

where $[\ldots]$ denotes the product of the nonrelativistic deuteron wave function, relativistic correction factors, and the scattering amplitude, or their spin-dependent generalizations. All threemomenta refer to the deuteron rest frame. $\boldsymbol{p}_{p}$ and $\boldsymbol{p}_{h}$ are the final-state proton and hadron momenta; $\boldsymbol{p}_{p 1}$ is the initial proton momentum and is the integration variable. The integration extends over the two-dimensional phase space allowed by energy and momentum conservation (on-shell rescattering). In the delta function, $p_{p}, p_{h}$ and $p_{p 1}$ are the four-momenta of the particles on their mass shells,

$$
\left.\begin{array}{rlrl}
p_{p} & =\left(E_{N}\left(\boldsymbol{p}_{p}\right), \boldsymbol{p}_{p}\right), & E_{N}\left(\boldsymbol{p}_{p}\right) & =\sqrt{\left|\boldsymbol{p}_{p}\right|^{2}+M_{N}^{2}} \\
p_{h} & =\left(E_{h}\left(\boldsymbol{p}_{h}\right), \boldsymbol{p}_{h}\right), & E_{h}\left(\boldsymbol{p}_{h}\right) & =\sqrt{\left|\boldsymbol{p}_{h}\right|^{2}+M_{h}^{2}} \\
p_{p 1} & =\left(E_{N}\left(\boldsymbol{p}_{p 1}\right), \boldsymbol{p}_{p 1}\right), & E_{N}\left(\boldsymbol{p}_{p 1}\right) & =\sqrt{\left|\boldsymbol{p}_{p 1}\right|^{2}+M_{N}^{2}}
\end{array}\right\},
$$

and the delta function enforces the mass shell condition for the the initial hadron four-momentum. We introduce the total four-momentum of the final proton-hadron system,

$$
\begin{aligned}
P & \equiv p_{p}+p_{h}, \quad P=\left(P^{0}, \boldsymbol{P}\right), \\
P^{0} & =E_{N}\left(\boldsymbol{p}_{p}\right)+E_{h}\left(\boldsymbol{p}_{h}\right), \quad \boldsymbol{P}=\boldsymbol{p}_{p}+\boldsymbol{p}_{h},
\end{aligned}
$$

whose square is the invariant mass of the proton-hadron system,

$$
s_{p h} \equiv P^{2} .
$$

In terms of this variable the argument of the delta function in Eq. (D1) becomes

$$
\begin{aligned}
\left(P-p_{p 1}\right)^{2}-M_{h}^{2}= & -2\left(P p_{p 1}\right)+P^{2}+M_{N}^{2}-M_{h}^{2} \\
= & 2\left|\boldsymbol{P} \| \boldsymbol{p}_{p 1}\right| \cos \chi-2 P^{0} E_{N}\left(\boldsymbol{p}_{p 1}\right) \\
& +P^{2}+M_{N}^{2}-M_{h}^{2}
\end{aligned}
$$

where $\chi$ is the angle between the three-momenta $\boldsymbol{p}_{p 1}$ and $\boldsymbol{P}$. For a given magnitude $\left|\boldsymbol{p}_{p 1}\right|$ the delta function fixes the value 
of $\cos \chi$ to be

$$
\cos \chi=\frac{2 P^{0} E_{N}\left(\boldsymbol{p}_{p 1}\right)-P^{2}-M_{N}^{2}+M_{h}^{2}}{2\left|\boldsymbol{P} \| \boldsymbol{p}_{p 1}\right|} \equiv X .
$$

A solution exists if $X^{2}<1$, which implies

$$
{ }_{4}|\boldsymbol{P}|^{2}\left|\boldsymbol{p}_{p 1}\right|^{2}-\left[2 P^{0} E_{N}\left(\boldsymbol{p}_{p 1}\right)-P^{2}-M_{N}^{2}+M_{h}^{2}\right]^{2}>0 .
$$

This condition is satisfied if

$$
\begin{aligned}
p_{\text {min }} & <\left|\boldsymbol{p}_{p 1}\right|<p_{\max }, \\
p_{\text {min }, \max } & \equiv \sqrt{\left(E_{\min , \max }\right)^{2}-M_{N}^{2}}, \\
E_{\min , \max } & \equiv \frac{1}{2 P^{2}}\left[P^{0}\left(P^{2}+M_{N}^{2}-M_{h}^{2}\right) \mp|\boldsymbol{P}| \sqrt{\lambda}\right], \\
\lambda & \equiv \lambda\left(P^{2}, M_{N}^{2}, M_{h}^{2}\right) \\
& =\left(P^{2}+M_{N}^{2}-M_{h}^{2}\right)^{2}-4 P^{2} M_{N}^{2} .
\end{aligned}
$$

Altogether, including the Jacobian factor, the phase space integral of Eq. (D1) becomes

$$
I=\left.\frac{1}{16 \pi^{2}|\boldsymbol{P}|} \int_{p_{\min }}^{p_{\max }} \frac{d p_{p 1} p_{p 1}}{E_{N}\left(\boldsymbol{p}_{p 1}\right)} \int_{0}^{2 \pi} d \phi[\ldots]\right|_{\cos \chi=X} .
$$

Here $p_{p 1} \equiv\left|\boldsymbol{p}_{p 1}\right|$, and $\phi$ is an angle specifying the direction of $\boldsymbol{p}_{p 1}$ in the plane perpendicular to the external vector $\boldsymbol{P}$. In particular, the integral with unit integrand (phase space) becomes

$$
\Gamma=\frac{\sqrt{\lambda}}{8 \pi s_{p h}}=\frac{p_{\mathrm{cm}}}{4 \pi \sqrt{s_{p h}}}, \quad p_{\mathrm{cm}} \equiv \frac{\sqrt{\lambda}}{2 \sqrt{s_{p h}}},
$$

as it should be ( $p_{\mathrm{cm}}$ is the $\mathrm{CM}$ momentum of the proton-hadron system).

The phase space integral Eq. (D1) extends over proton momenta $\left|\boldsymbol{p}_{p 1}\right| \ll M_{N}$, where the wave function in the inte- grand $[\ldots]$ is described by the nonrelativistic approximation. In this situation the proton energy $E_{N}\left(\boldsymbol{p}_{p 1}\right)$ in the measure of Eq. (D13) can be replaced by the nucleon mass $M_{N}$. Note that no assumption regarding the hadron momentum $\left|\boldsymbol{p}_{h}\right|$ was made in resolving the phase space delta function; the above formulas are valid for $\left|\boldsymbol{p}_{h}\right| \sim M_{h}$ and can be used over the entire relevant range of hadron momenta.

When evaluating the rescattering integral with the deuteron wave function in the nonrelativistic approximation, the integrand [...] generally depends on the direction of the vector $\boldsymbol{p}_{p 1}$. For example, the relativistic correction factor in Eq. (6.50) is proportional to $p_{p 1}^{z}$; and the $D$-wave component involves the tensor product $p_{p 1}^{i} p_{p 1}^{j}$. These vector/tensor valued integrals can conveniently be computed by expanding the vector $\boldsymbol{p}_{p 1}$ in an orthogonal basis constructed from the external vectors $\boldsymbol{p}_{p}$ and $\boldsymbol{p}_{h}$. We choose the basis vectors as

$$
\begin{aligned}
& \{\boldsymbol{P}, \boldsymbol{\Delta}, \boldsymbol{N}\} ; \quad \boldsymbol{P}=\boldsymbol{p}_{p}+\boldsymbol{p}_{h}, \\
& \Delta \equiv \boldsymbol{p}_{p}-\frac{\left(\boldsymbol{p}_{p} \boldsymbol{P}\right) \boldsymbol{P}}{|\boldsymbol{P}|^{2}}, \quad \boldsymbol{N} \equiv \Delta \times \boldsymbol{P},
\end{aligned}
$$

and write

$$
\boldsymbol{p}_{p 1}=p_{p 1}\left(\cos \chi \frac{\boldsymbol{P}}{|\boldsymbol{P}|}+\sin \chi \cos \phi \frac{\boldsymbol{\Delta}}{|\boldsymbol{\Delta}|}+\sin \chi \sin \phi \frac{\boldsymbol{N}}{|\boldsymbol{N}|}\right)
$$

such that the angle $\phi$ is measured relative to the $\Delta$ direction in the plane orthogonal to $\boldsymbol{P}$. This expansion allows one to convert the original vector/tensor integrals into angular integrals, which multiply vectors/tensors formed from the basis vectors. The components of the latter in the collinear frame $(x, y, z)$ can be calculated from the LF momentum components $\alpha_{p}, \boldsymbol{p}_{p T}$ and $\alpha_{h}, \boldsymbol{p}_{p T}$ (see Sec. IID).
[1] L. L. Frankfurt and M. I. Strikman, Phys. Rep. 160, 235 (1988).

[2] M. Arneodo, Phys. Rep. 240, 301 (1994).

[3] L. Frankfurt, V. Guzey, and M. Strikman, Phys. Rep. 512, 255 (2012).

[4] S. Malace, D. Gaskell, D. W. Higinbotham, and I. Cloet, Int. J. Mod. Phys. E 23, 1430013 (2014).

[5] D. Boer et al., arXiv:1108.1713.

[6] A. Accardi et al., Eur. Phys. J. A 52, 268 (2016).

[7] US Department of Energy Office of Science Nuclear Science Advisory Committee (NSAC) 2015 Long Range Plan for Nuclear Science, http: //science.energy .gov/np/nsac

[8] A. Accardi, V. Guzey, A. Prokudin, and C. Weiss, Eur. Phys. J. A 48, 92 (2012).

[9] S. Abeyratne et al., arXiv:1209.0757.

[10] A. J. Baltz et al., Phys. Rep. 458, 1 (2008).

[11] L. L. Frankfurt and M. I. Strikman, Phys. Rep. 76, 215 (1981).

[12] L. Frankfurt, V. Guzey, and M. Strikman, Phys. Lett. B 381, 379 (1996).
[13] F. R. P. Bissey, V. A. Guzey, M. Strikman, and A. W. Thomas, Phys. Rev. C 65, 064317 (2002).

[14] C. Boros, V. A. Guzey, M. Strikman, and A. W. Thomas, Phys. Rev. D 64, 014025 (2001).

[15] M. Sargsian and M. Strikman, Phys. Lett. B 639, 223 (2006).

[16] S. Tkachenko et al. (CLAS Collaboration), Phys. Rev. C 89, 045206 (2014); 90, 059901(E) (2014).

[17] K. A. Griffioen, J. Arrington, M. E. Christy, R. Ent, N. Kalantarians, C. E. Keppel, S. E. Kuhn, W. Melnitchouk, G. Niculescu, I. Niculescu, S. Tkachenko, and J. Zhang, Phys. Rev. C 92, 015211 (2015).

[18] S. Bueltmann et al., Jefferson Lab $12 \mathrm{GeV}$ Experiment E1206-113, https://www.jlab.org/exp_prog/proposals/ 06/PR12-06-113.pdf

[19] E. C. Aschenauer et al., arXiv:1409.1633.

[20] JLEIC Main Public Wiki Page: https://eic.jlab. org/wiki/

[21] eRHIC Main Page: https://wiki.bnl.gov/eic/

[22] V. Guzey, M. Strikman, and C. Weiss (unpublished). 
[23] A. Arvidson et al. (European Muon Collaboration), Nucl. Phys. B 246, 381 (1984).

[24] J. Ashman et al. (European Muon Collaboration), Z. Phys. C 52, 1 (1991).

[25] M. R. Adams et al. (E665 Collaboration), Z. Phys. C 61, 179 (1994).

[26] M. R. Adams et al. (E665 Collaboration), Phys. Rev. Lett. 74, 5198 (1995); 80, 2020(E) (1998).

[27] A. Airapetian et al. (HERMES Collaboration), Eur. Phys. J. C 20, 479 (2001).

[28] A. Airapetian et al. (HERMES Collaboration), Nucl. Phys. B 780, 1 (2007).

[29] M. Strikman, M. G. Tverskoii, and M. B. Zhalov, Phys. Lett. B 459, 37 (1999).

[30] L. Trentadue and G. Veneziano, Phys. Lett. B 323, 201 (1994).

[31] J. C. Collins, Phys. Rev. D 57, 3051(E) (1998); 61, 019902 (2000)

[32] P. A. M. Dirac, Rev. Mod. Phys. 21, 392 (1949).

[33] H. Leutwyler and J. Stern, Ann. Phys. 112, 94 (1978).

[34] F. Coester, Prog. Part. Nucl. Phys. 29, 1 (1992).

[35] S. J. Brodsky, H. C. Pauli, and S. S. Pinsky, Phys. Rep. 301, 299 (1998).

[36] T. Heinzl, Habilitation thesis, Regensburg University, 1998, arXiv:hep-th/9812190.

[37] W. Cosyn and M. Sargsian, Phys. Rev. C 84, 014601 (2011).

[38] W. Cosyn, W. Melnitchouk, and M. Sargsian, Phys. Rev. C 89, 014612 (2014).

[39] W. Cosyn and M. Sargsian, Int. J. Mod. Phys. E 26, 1730004 (2017).

[40] W. Cosyn, M. Sargsian, and C. Weiss (unpublished).

[41] L. L. Frankfurt and M. I. Strikman, Nucl. Phys. A 405, 557 (1983).

[42] C. Ciofi degli Atti and B. Z. Kopeliovich, Eur. Phys. J. A 17, 133 (2003).

[43] C. Ciofi degli Atti, L. P. Kaptari, and B. Z. Kopeliovich, Eur. Phys. J. A 19, 145 (2004).

[44] V. Palli, C. Ciofi degli Atti, L. P. Kaptari, C. B. Mezzetti, and M. Alvioli, Phys. Rev. C 80, 054610 (2009).

[45] C. Ciofi degli Atti and L. P. Kaptari, Phys. Rev. C 83, 044602 (2011).

[46] L. L. Frankfurt, W. R. Greenberg, G. A. Miller, M. M. Sargsian, and M. I. Strikman, Z. Phys. A 352, 97 (1995).

[47] W. Boeglin and M. Sargsian, Int. J. Mod. Phys. E 24, 1530003 (2015).

[48] V. B. Berestetskii, E. M. Lifshitz, and L. P. Pitayevskii, in Relativistic Quantum Theory, Course of Theoretical Physics Vol. IV (Pergamon, Oxford, 1973).

[49] R. K. Ellis, W. Furmanski, and R. Petronzio, Nucl. Phys. B 212, 29 (1983).

[50] S. Weinberg, Phys. Rev. 150, 1313 (1966).

[51] J. R. Cooke and G. A. Miller, Phys. Rev. C 66, 034002 (2002).

[52] J. R. Cooke, Ph.D. thesis, University of Washington, 2001.

[53] G. A. Miller and R. Machleidt, Phys. Rev. C 60, 035202 (1999).

[54] G. A. Miller, Prog. Part. Nucl. Phys. 45, 83 (2000).
[55] M. V. Terentev, Sov. J. Nucl. Phys. 24, 106 (1976) [Yad. Fiz. 24, 207 (1976)].

[56] R. B. Wiringa, V. G. J. Stoks, and R. Schiavilla, Phys. Rev. C 51, 38 (1995).

[57] J. B. Kogut and D. E. Soper, Phys. Rev. D 1, 2901 (1970).

[58] J. M. Cornwall and R. Jackiw, Phys. Rev. D 4, 367 (1971).

[59] O. Benhar, N. Farina, H. Nakamura, M. Sakuda, and R. Seki, Phys. Rev. D 72, 053005 (2005).

[60] C. Ciofi degli Atti, L. L. Frankfurt, L. P. Kaptari, and M. I. Strikman, Phys. Rev. C 76, 055206 (2007).

[61] N. Christ and T. D. Lee, Phys. Rev. 143, 1310 (1966).

[62] A. Afanasev, M. Strikman, and C. Weiss, Phys. Rev. D 77, 014028 (2008).

[63] K. M. Hanson, Cornell University Laboratory of Nuclear Studies Report CLNS-317.

[64] M. Arneodo et al. (European Muon Collaboration), Phys. Lett. B 149, 415 (1984).

[65] M. Arneodo et al. (European Muon Collaboration), Phys. Lett. B 150, 458 (1985).

[66] M. Arneodo et al. (European Muon Collaboration), Z. Phys. C 31, 1 (1986).

[67] C. Alexa et al. (H1 Collaboration), Eur. Phys. J. C 73, 2406 (2013)

[68] V. Andreev et al. (H1 Collaboration), Eur. Phys. J. C 74,2915 (2014).

[69] F. D. Aaron et al. (H1 Collaboration), Eur. Phys. J. C 68, 381 (2010)

[70] S. Chekanov et al. (ZEUS Collaboration), J. High Energy Phys. 06 (2009) 074.

[71] M. Derrick et al., Phys. Rev. D 17, 1 (1978).

[72] P. Allen et al. (Aachen-Bonn-CERN-Munich-Oxford Collaboration), Nucl. Phys. B 188, 1 (1981).

[73] P. Allen et al. (Aachen-Bonn-CERN-Munich-Oxford Collaboration), Nucl. Phys. B 214, 369 (1983).

[74] A. Arriaga and R. Schiavilla, Phys. Rev. C 76, 014007 (2007).

[75] C. Granados and C. Weiss, J. High Energy Phys. 07 (2015) 170.

[76] G. Schierholz, Nucl. Phys. B 7, 432 (1968).

[77] F. Coester, Helv. Phys. Acta 38, 7 (1965).

[78] J. M. Namyslowski, Phys. Rev. D 18, 3676 (1978).

[79] W. Cosyn et al., J. Phys. Conf. Ser. 543, 012007 (2014).

[80] V. Guzey, D. Higinbotham, C. Hyde, P. Nadel-Turonski, K. Park, M. Sargsian, M. Strikman, and C. Weiss, PoS DIS 2014, 234 (2014), arXiv:1407.3236 [hep-ph].

[81] C. Weiss et al., Jefferson Lab 2014/15 Laboratory-directed R\&D Project, https://www.jlab.org/theory/tag/

[82] W. Melnitchouk, M. Sargsian, and M. I. Strikman, Z. Phys. A 359, 99 (1997).

[83] O. Hen, L. B. Weinstein, S. Gilad, and S. A. Wood, arXiv:1409.1717.

[84] G. Wolf, Rep. Prog. Phys. 73, 116202 (2010).

[85] C. Lechanoine-LeLuc and F. Lehar, Rev. Mod. Phys. 65, 47 (1993).

[86] B. H. Silverman et al., Nucl. Phys. A 499, 763 (1989).

[87] A. V. Dobrovolsky et al., Nucl. Phys. B 214, 1 (1983). 